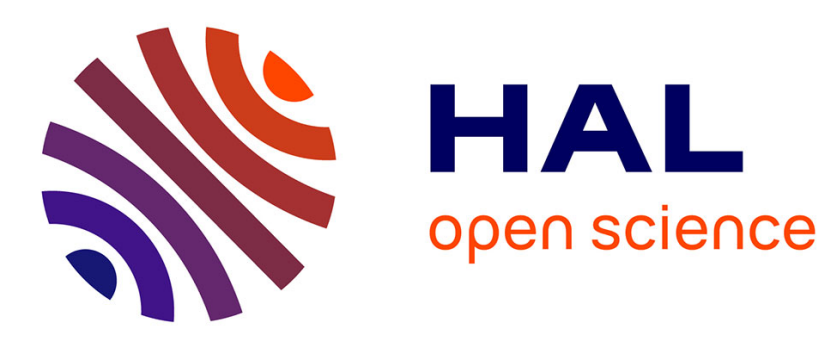

\title{
Charge trap spectroscopy in polymer dielectrics: a critical review
}

\author{
G. Teyssedre, Feihu Zheng, Laurent Boudou, Christian Laurent
}

\section{To cite this version:}

G. Teyssedre, Feihu Zheng, Laurent Boudou, Christian Laurent. Charge trap spectroscopy in polymer dielectrics: a critical review. Journal of Physics D: Applied Physics, 2021, 54 (26), pp.263001. 10.1088/1361-6463/abf44a . hal-03326106

\section{HAL Id: hal-03326106 https://hal.science/hal-03326106}

Submitted on 25 Aug 2021

HAL is a multi-disciplinary open access archive for the deposit and dissemination of scientific research documents, whether they are published or not. The documents may come from teaching and research institutions in France or abroad, or from public or private research centers.
L'archive ouverte pluridisciplinaire HAL, est destinée au dépôt et à la diffusion de documents scientifiques de niveau recherche, publiés ou non, émanant des établissements d'enseignement et de recherche français ou étrangers, des laboratoires publics ou privés. 


\title{
Charge Trap Spectroscopy in Polymer Dielectrics:
}

\section{A Critical ReVieW}

\author{
Gilbert Teyssedre $^{1,2^{*}}$, Feihu Zheng ${ }^{2}$, Laurent Boudou ${ }^{1}$, Christian Laurent $^{1}$ \\ ${ }^{1}$ Laplace, CNRS and University of Toulouse, Toulouse, France \\ ${ }^{2}$ Department of Electrical Engineering, Tongji University, Shanghai, China \\ *gilbert.teyssedre@laplace.univ-tlse.fr
}

\begin{abstract}
Trapping phenomena are essential features controlling the transport properties of insulating materials. Depending on the energy depth, traps can either assist transport or lead to long-lasting storage of charges. The consequences of charge trapping are non-linear phenomena and electric field distribution distortion in the dielectric bulk. The important characteristics about traps are the nature of the levels, their depth in energy, and their density. In this review, we discuss the different techniques available to probe the energetics of traps, particularly in insulating polymers. The methods implemented for approaching the characteristics of traps range from atomistic simulation based on known physical/chemical defects, identification by spectroscopic techniques, and coupled opticalelectrical or thermal-electrical techniques. The review is focused on methods involving thermal or optical excitation coupled to detection using electrical or luminescence response with questioning about the physical hypotheses behind the analysis and the difference in response obtained through the various approaches. The technical implementation of these methods is described, along with examples of application. The differences in trap depth estimation from optical and thermal methods is discussed as well as the impact of having distributed trap depths. The input of luminescence techniques, which provide a fingerprint of chemical groups involved in charge recombination, is put forward.
\end{abstract}

Keywords: polymer dielectrics, trapping phenomena, thermal excitation, electro-optical effects, space charge 


\section{Introduction}

The electrical properties of insulating materials, especially electrical transport and breakdown are most often described by concepts issued from semiconductor physics that are further adapted [1, 2]. Adaptation consists, for polymer insulators, in accounting for the disordered nature of materials. Important fundamental processes that have been treated in this way are hopping conduction $[3,4,5]$ space charge control of the current [6,7], mediation of injection processes by interfacial states [8], long lasting charge storage, etc. One of the consequences of such phenomenon is space charge buildup, i.e. local non-neutrality, leading to a poor control of the electric field, and non-linear macroscopic behavior for conductivity. Besides stabilizing charges and depending on the depth of the energetic levels, trapping phenomena control transport through for example shallow states and promotion of charge injection at the electrodes.

The salient question in there is on the description of the energetic levels providing the specific properties. This is even more challenging as the amorphous or partly amorphous nature of polymers is combined to a chemical complexity and 'defects' cannot be singled out.

In this review, we start recalling three fundamental quantities related to traps, i.e. their nature, energy and density. Trap spectroscopy has the objective to gather a maximum of information about these quantities but none of the techniques used so far is able to give a complete picture and one of the outlook is certainly to combine different techniques to get a full image. We continue by giving an overview of the different methods and the pre-charging step, trying to clarify the landscape by distinguishing between the techniques that do not involve a stimulation for charge detrapping from those that do. Among the first category, surface potential decay and space charge decay are quite familiar unlike charge recombination-induced luminescence. The two former ones lead to trap density distribution but rely on numerous hypotheses that are hardly fulfilled. The latter one provides an elegant way to infer the chemical nature of trapping centers. We then visit the methods that involve a stimulation for charge detrapping being thermal or optical, the measured quantity being current or luminescence. The discussion section treats some important questions linked to the intrinsic nature of polymers where the overlapping of distributed processes complexifies the interpretation of results from thermally stimulated methods. The notion of trap depth is also discussed in regards of relaxation phenomena involving the physical disappearance of the traps, at relaxation temperatures that correspond to the onset of local molecular motions of the polymer. Discrepancies also exist between thermal and optical techniques when considering the depth of traps and this question has not found a definitive answer up to now. Luminescence techniques are finally considered being able to identify the chemical nature of trapping centers. Some ways to go forward for a better understanding of traps are introduced in the summary. 


\section{Traps in polymer dielectrics}

The kind of defects can be to some extent put in relation to their impact on properties. Physical disorder encompasses all conformational disorder forming the amorphous phase and its interface with the crystalline part. In this respect, this is essentially through modelling that the properties can be approached. The work carried out so far has been mainly on polyethylene. Disorder introduces shallow trapping level, also designed as Anderson or mobility levels [9] at a depth of up to about $0.3 \mathrm{eV}$ (this separation between extended states from localized states is also termed mobility edge) in amorphous polyethylene [10]. Because of the relative shallow nature of such traps, and high density, their role is mainly a boost for electron transport. With a large density of localized states in interaction, carriers can tunnel from one level to another one without undergoing a transition to the extended states. Hole transport has been investigated as well. As an example, Unge and Christen investigated the hole and electron mobility edges by molecular simulation, confirming that hole transport in LDPE could be dominant [11]. Experimental verification of such state characteristics cannot be easily realized other than by very macroscopic measurements like activation energy estimation for conduction. Here the strict correlation to a single activated process is not verified since transport is often bipolar and other processes are thermally activated as well, notably charge injection [12].

Asides these shallow energy levels representing a form of continuum, deep trapping centers have been identified corresponding to chemical defects. In polymers, all sorts of chemical disorder can be thought of, going from side chains, unsaturations, oxidized groups to additives, to reaction products and to impurities. The chemical disorder is thought to control the space charge, and these energy levels are often named deep traps, or "real traps" because their contribution to the conduction process is low, as opposed to shallow traps that assist conduction [2]. The energy depth associated with such traps is generally larger than for physical traps, but not systematically. For polyethylene, trap depths in the range from 0.1 to $1.6 \mathrm{eV}$ have been reported by molecular modelling for selected defects [13]. Also, a given defect can be a trap for electrons and / or holes. The main effect of such levels is thought not to contribute to transport since their density is much less than that of transport states. Rather, the detrimental effect is to stabilize charges and contribute to the field redistribution: this can then indirectly affect the apparent conductivity, notably through a modulation of the field at the interface as well as the transport in the bulk owing to the non-linear phenomena introduced by shallow traps. Deep trapping of charges also introduces hysteresis phenomena in the response of the material. Ultimately, it is exploited in electrets - those materials capable of long-lasting storage of charges to realize sensors and actuators [14]. However, for the case of insulators under DC stress it represents a threat [15] since it goes with redistribution of the electric field and to strong local field enhancement at polarity reversal. The energy released by carrier detrapping and recombination with carriers of opposite polarity is a further process considered as a possible long term ageing route [16].

It is therefore important to find ways to identify traps into materials. Three essential features are to be identified:

\subsection{The nature of trap}

The question is on the nature in terms of chemicals/point defect. As stated previously, many different kinds of defects are present into polymers and are candidates as traps. However, their efficiency and role can be very variable, depending on the energy level they generate and on the capture cross- 
section for carriers. The first level of experiments can be the chemical analysis of 'defects'. In Polyethylene materials, the in-chain or side-chain imperfections, i.e. hydroxyl and ketone functions, double or triple carbon-carbon bonds, branching and so on constitute the first level. Some examples for such chemical groups [17] are given in Figure 1. In peroxide-crosslinked polyethylene materials, byproducts constitute a second family. The role of such in chain effects and residues has been relatively well explored by molecular simulation [13, 18, 19]. Further candidates are antioxidant and related reaction products [20]. The difficulty in the plethora of candidates is to identify which ones are active, i.e. exhibit an effective capture cross-section. So, methods addressing specifically the propensity of defects to store charges are looked: recombination-induced luminescence [21] is one of them and we shall illustrate its potentialities in the following.

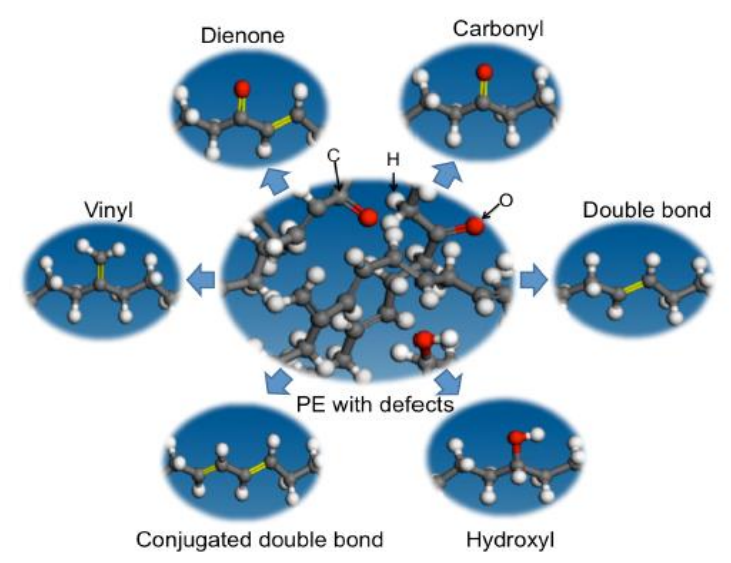

Figure 1. Some in-chain defects in Polyethylene. Reprinted from Chen et al [17] with permission from AIP Publishing.

\subsection{The energy associated with the trap}

Molecular modelling is one of the ways the trap energy can be anticipated. On the experimental side, different methods have been implemented, consisting in pre-charging of the material using different sources that can be ionizing radiation, corona discharge, application of a high DC field, electron irradiation, etc. Then, the charges are released by either thermal or optical excitation as illustrated in Figure 2 . The energy necessary to release the charges is assimilated as the trap depth.

The external current is most often used as a probe of charge release. In case of thermal excitation the technique is called thermally stimulated discharge current (TSDC) $[22,23,24]$; which has been combined to thermally stimulated luminescence (TL) [25]. Current recording following optical excitation is called photostimulated discharge (PSD) $[26,27,28]$. In the simplest of the cases, the energy provided to the charges to overpass the barrier to conduction levels is considered as the trap level. However, as will be shown later on, other processes may be active and the access to the trap energy distribution is not straightforward.

Besides these two methods relying on the collection of the current upon charge release, charge detrapping can be indirectly analyzed through luminescence measurements reflecting the radiative recombination of charges that may follow the detrapping, as depicted in Figure 2. 


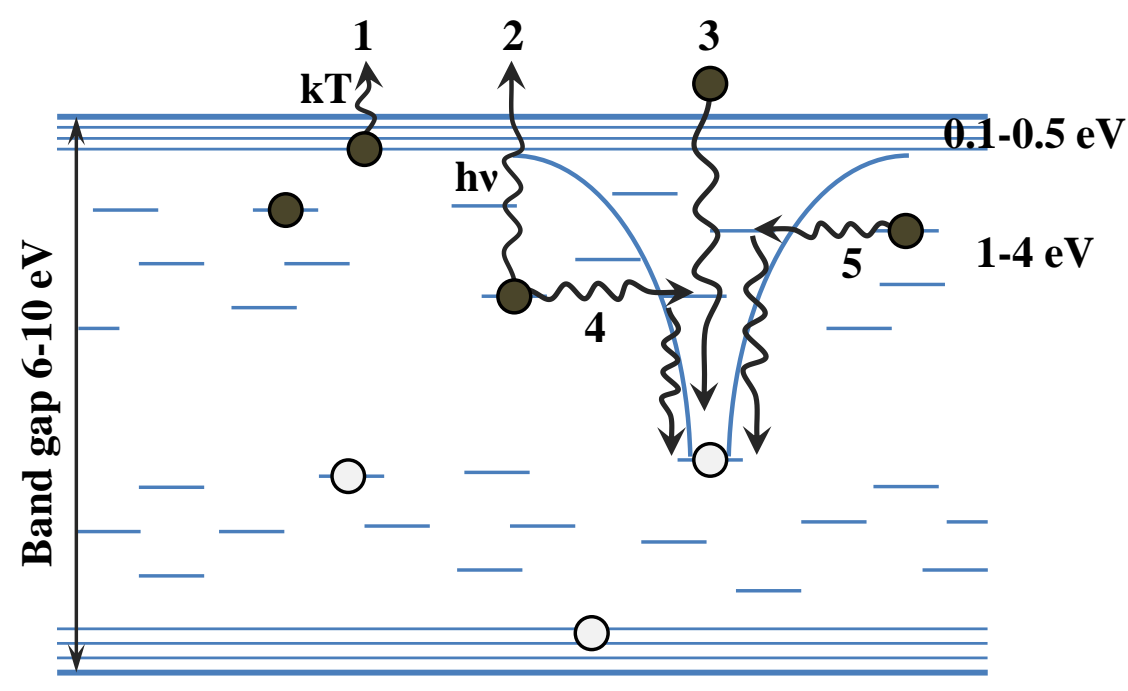

Figure 2. Processes involved in (deep) trap spectroscopy techniques on wide bandgap materials: (1) thermal detrapping; (2) optical detrapping; (3) simple charge recombination; $(1+3)$ thermally activated recombination; $(4,5)$ charge recombination by resonant tunneling with coupling to an excited state of the recombining center.

There are some advantages in analyzing charge recombination as there is no need to account for charge transport to get a signal, but the kinetics is more complex and the qualitative and quantitative nature of the response is depending on the recombining center.

\subsection{The density of traps}

The work carried out using chemical analyses in principle can provide density of potential traps if the analysis is quantitative. It is however not easy to anticipate the efficiency of a given moiety to act as a trap, as stated above. Alternatively, techniques such as TSDC, which are applied once the material has been previously charged, may give information on the amount of charges, i.e. of occupied traps. Inferring the total amount of traps supposes that a model linking the population of traps with the detrapping rate from the occupied traps be available, which is not obvious. Quantitative information on stored charge density can also be obtained from space charge distribution measurements, but again the stored net charge is obtained, not the maximum density at trap saturation.

An order of magnitude of trap density can be given considering the first approach (analytical) combined with molecular modelling [13, 29], cf. Figure 3. Here physical and chemical trap densities were estimated as a function of the energy depth for low-density polyethylene. The approach has been used to draw a continuous exponential distribution of trap depths as represented in Figure 3 [30]. Here, whereas physical traps could be considered as exhaustively accounted for, only some of the chemical traps, constituted of insaturations and oxidized groups have been analyzed [18]. A much broader family of chemicals should be investigated. Taking as $10^{18} \mathrm{~cm}^{-3}$ an order of magnitude of trap density with depth larger than $0.8 \mathrm{eV}$, then stored charge density $>10^{5} \mathrm{C} / \mathrm{m}^{3}$ would be obtained at trap saturation. This is much larger than most of measurements reported so far using injecting electrodes. One of the reasons is that the injection field is reduced by the already injected charge. 


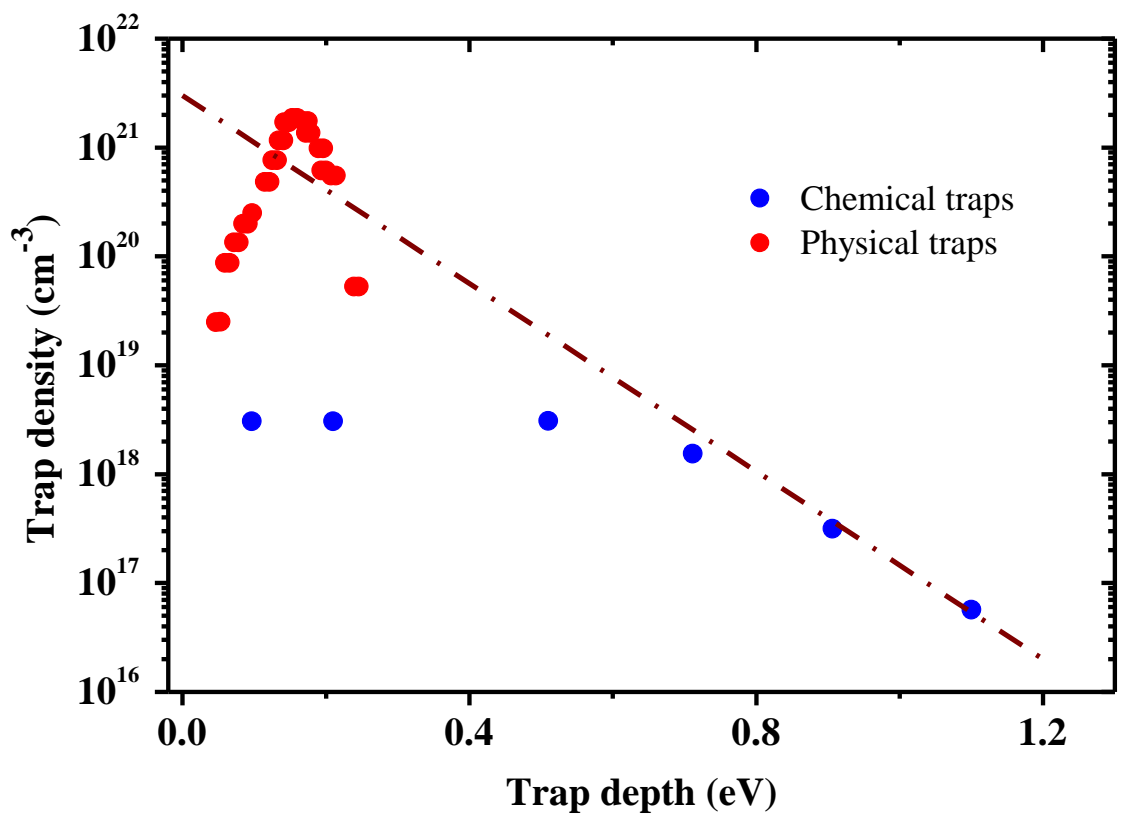

Figure 3. Discrete distribution of electron traps density as a function of trapping energy. The circles are molecular simulations results [13]. It has been approximated to a continuous exponential distribution function represented as dash-dot line. Reprinted from [30], with the permission of AIP Publishing.

Only experiments using focused e-beam for charging reported on charge densities of the order of $\mathrm{MC} / \mathrm{m}^{3}$ [31] and then the limit in charge amount would be the breakdown field. It means that, in general, a priori only a very small fraction of traps is occupied, or charges are trapped in a non-uniform way and the measurement "averages" their density over the spatial resolution of the experiments.

The above features have parallel effects in organic semiconductors: traps are inherent to organic materials owing to the weak intermolecular interactions. Trapping has profound impact on device performances [32]. Methods to probe it are however somewhat different as layers are usually thinner, charge mobility much higher and trap states less deep compared to insulators.

In the following, we review the different techniques available to approach one or several of the characteristics described above. Shallow or 'physical traps' forming a nearly continuum of states do participate in transport without contributing to long lasting charge trapping. Besides, there is no technique easily implementable to probe their density. For these reasons, we will focus here mainly on the relatively deep traps induced by chemical defects. 
Review: Trap Spectroscopy in Polymer Dielectrics - Revised Version.

\section{Overview of experimental techniques for trap spectroscopy}

The characteristics of techniques addressed in this work are summarized in Table 1. The methods are based on natural decay of potential or charge, or on either optical or thermal excitation. The detection is either with electrical or optical signals. For each of the method, the nature of information gained, advantage and drawbacks are given and supported by the description given in the following sections.

\subsection{Families of methods}

As a way of sorting the methods, we have chosen to introduce the distinction between techniques that need an excitation vs. those that don't need. The analysis of the natural decay of the charge constitutes one way for approaching the charge traps characteristics. This constitutes a first family of techniques in which isothermal charge decay [33, 34, 35], and more largely surface potential decay [36, 37, 38, 39 ] is employed. The change in potential vs. time is related to the release of charges from traps and their drift in the insulation under the field produced by the charges. As the information gained is integral, hypotheses have to be taken about the nature of charges and the charge release processes, as well as on the processes at the charging step [40]. These aspects will be treated in section 4.1.

In recent years, with the development of charge distribution measurement techniques, the analysis of the kinetics of charge release has been refined in order to provide information on the distribution of trap depths endowing the charge release. The experiments then provide insights into hypotheses taken for the macroscopic surface potential decay. The analysis has been initiated by Dissado et al. [41], and refined with considering different hypotheses on the charge configuration $[42,43]$.

Optical emission in electrically stressed polymers has been related to space charge effects and has been used as a proof of energy release to investigate the dissipation mechanisms under field [44]. Among the various ways of exciting light, charge-recombination induced luminescence (RIL) consists in analyzing the light decay following bipolar charging of the material [21, 45]. Here the information gained is mostly on the mechanisms of charge recombination: the nature of chemical groups involved and the kinetics of charge release without requiring their transport. Luminescence has previously been applied to a variety of pre-irradiation methods, particularly with $\gamma$-rays $[46,47]$. The specificity of the method we will describe here is that the surface of the material only is charged.

The last three methods addressed consider the response of a pre-charged material to a stimulated discharge using either optical or thermal excitation. We will discuss the fundamental differences between the two excitation ways and the reasons for the differences in obtained trap distributions.

Scanning with optical excitation is expected to provide directly an estimate of the trap depth corresponding to the onset of current increase when increasing the light energy (Figure 2, path 2). However, we shall see in section 5.3 that other processes active in photoconduction may contribute to the response [48]. In the case of thermal stimulation (TSDC or TL), a kinetic analysis is necessary. This may not be straightforward in case of distributed processes [49] as discussed in section 6.1. When the response is a collected current, as in TSDC, it corresponds to the space-averaged transport current in short-circuit conditions. It is therefore sensitive to the initial position of detrapped charges and to their fate along the path to the collecting electrode. This makes a difference with TL than involves charge recombination phenomena without long-range transport. We discuss how the information gained by TL can be exploited complementarily to TSDC [50] as it probes recombination events and the chemical nature of recombination centers. 
Review: Trap Spectroscopy in Polymer Dielectrics - Revised Version. Teyssedre et al 2021 J. Phys. D: Appl. Phys. https://doi.org/10.1088/1361-6463/abf44a

\begin{tabular}{|c|c|c|}
\hline \multirow{6}{*}{ 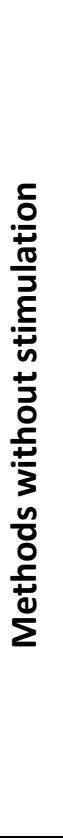 } & \multicolumn{2}{|c|}{ Surface potential decay } \\
\hline & $\begin{array}{l}\Rightarrow \text { Excitation: none } \\
\Rightarrow \text { Detection: surface potential } \\
\Rightarrow \text { Hypotheses: thermal activation of charges } \\
\text { from traps with no retrapping }\end{array}$ & $\begin{array}{l}\text { access to trap depth spectrum } \\
\text { difficulty in controlling the charging process } \\
\text { many hypotheses for the analysis }\end{array}$ \\
\hline & \multicolumn{2}{|c|}{ Decay of space charge distribution } \\
\hline & $\begin{array}{l}\Rightarrow \text { Excitation: none } \\
\Rightarrow \text { Detection: space charge density profile } \\
\Rightarrow \text { Hypotheses: thermal activation of charges } \\
\text { from traps with no retrapping }\end{array}$ & $\begin{array}{l}\text { access to trap depth spectrum } \\
\text { control of the initial charge distribution } \\
\text { (unipolar vs. bipolar) }\end{array}$ \\
\hline & \multicolumn{2}{|c|}{ Charge recombination - induced luminescence } \\
\hline & $\begin{array}{l}\Rightarrow \text { Excitation: none (isothermal) } \\
\Rightarrow \text { Detection: luminescence } \\
\Rightarrow \text { Hypotheses: thermal activation of charges } \\
\text { from traps with recombination on } \\
\text { recombining centers }\end{array}$ & $\begin{array}{l}\text { information on the nature of recombining } \\
\text { centers from the emission spectrum } \\
\text { no information on trap depth } \\
\text { quantitative analysis not easy with } \\
\text { luminescence }\end{array}$ \\
\hline \multirow{6}{*}{ 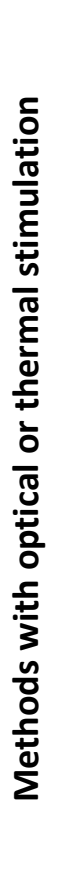 } & \multicolumn{2}{|c|}{ Photo-stimulated discharge } \\
\hline & $\begin{array}{l}\Rightarrow \text { Excitation: monochromatic light } \\
\Rightarrow \text { Detection: current (collecting V) } \\
\Rightarrow \text { Hypothesis: photo-detrapping of charges } \\
\text { from traps with no retrapping }\end{array}$ & $\begin{array}{l}\text { direct access to trap depth spectrum } \\
\text { coupled thermal excitation } \\
\text { sensitivity, quantitative aspects (transport) }\end{array}$ \\
\hline & \multicolumn{2}{|c|}{ Thermally stimulated currents } \\
\hline & $\begin{array}{l}\Rightarrow \text { Excitation: T ramp-up } \\
\Rightarrow \text { Detection: current } \\
\Rightarrow \text { Hypotheses: thermo-detrapping of charges } \\
\text { from traps with no retrapping }\end{array}$ & $\begin{array}{l}\text { sensitivity } \\
\text { trap depth from thermo-current analysis } \\
\text { thermal cycling } \\
\text { detrapping vs. trap destruction } \\
\text { analysis for distributed processes }\end{array}$ \\
\hline & \multicolumn{2}{|c|}{ Thermo-Iuminescence } \\
\hline & $\begin{array}{l}\Rightarrow \text { Excitation: T ramp-up } \\
\Rightarrow \text { Detection: luminescence } \\
\Rightarrow \text { Hypotheses: thermo-detrapping of charges } \\
\text { from traps with recombination on } \\
\text { recombining centers }\end{array}$ & $\begin{array}{l}\text { same as TSC } \\
\text { information from the TL emission spectrum } \\
\text { T-dependent luminescence quantum yield }\end{array}$ \\
\hline
\end{tabular}

Table 1. Main trap spectroscopy methods and associated characteristics.

\subsection{Pre-charging step}

Whatever the probing method, traps need to be populated for being probed. Different methods are used to create charges into the sample, and they substantially affect the way the response is analyzed and the information gained. We can distinguish homogeneous vs. localized charge deposition in the depth of the sample and unipolar vs. bipolar charging. 


\subsubsection{Surface charging}

A simple way of depositing charges consists in creating a discharge in the gas in the environment of the sample. A corona discharge under DC (Figure 4) will generate charges of desired polarity, in a controlled amount by controlling the surface potential. Electrons and ions are deposited; the monitoring of the potential decay following charging can be a way to analyze discharge kinetics and hence detrapping features. However, this is through a global approach and obtaining a spectrum in energy is not straightforward or requires severe hypotheses on the distribution. A missing link can be the mechanism whereby deposited ions transfer their charges to the material. As positive or negative ions are considered as the main source of charges, a charge exchange process is considered at the surface $[14,51]$. This leads to consider in modelling that the deposited charges are injected into the material in the course of the charge decay. Also, the discharge can possibly modify the surface properties of the material, notably with creating oxidized groups or oxidized molecules $[52,53]$.

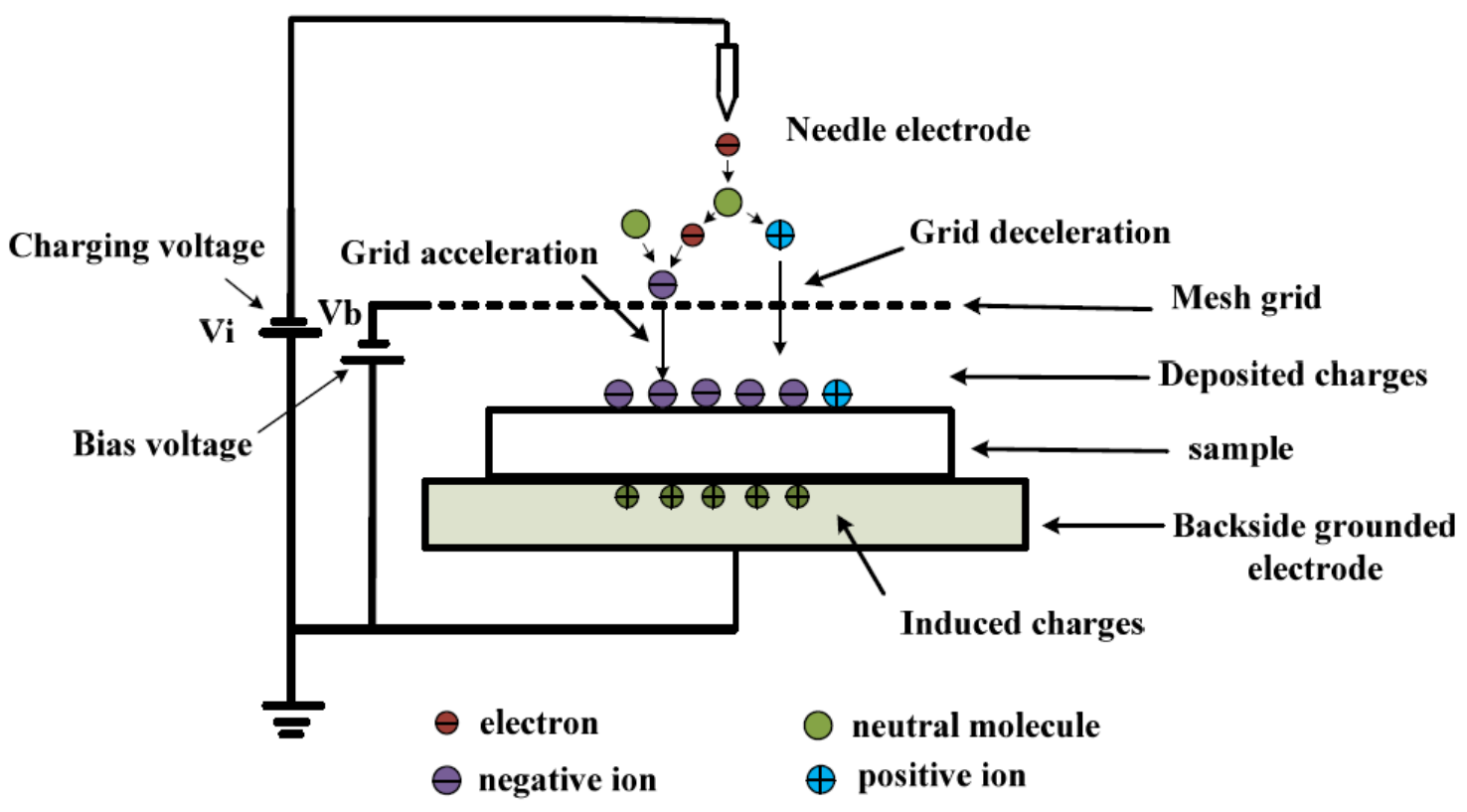

Figure 4. Principle of charging by corona discharge. The potential $\mathrm{Vb}$ set to the mesh grid is the steady state potential of the surface. Note that with a negative charging voltage, both electrons and negative ions are deposited.

Reprinted with permission from Li et al [39] Copyright 2015 IEEE.

Instead of applying a DC corona discharge, a homogeneous AC discharge can be created and then bipolar charging is achieved. By using mild RF discharge in Helium, it was shown that luminescence signal from both surface modification and radiative charge recombination could be obtained [45]. The control of the discharge limits surface modification. Activation of the material is presumably from the deposited charge by the plasma, but it was reported also that the UV from the discharge may activate as well [54], which tends to show that it is not only a surface analysis method. 


\subsubsection{Application of a DC field}

Charges can be created at the surface by applying a DC field to the sample provided with electrodes. In comparison to other methods, the charging (position, nature and amount of charges) is tributary on charge exchange processes at the electrode and on transport in the material bulk. In principle, it leads to bipolar charging with positive and negative charges generated at different positions and different amounts. Besides charges generation/motion, the field induces orientation of dipoles, which relaxation can contribute to the response besides charge detrapping. TSDC has long been used to probe such dipole relaxation to investigate dynamic processes in polymers and disordered media, the analysis of which being relatively more simple as the issues of charge transport and localization of the response need not to be handled $[55,56,57]$.

\subsubsection{Electron beam implantation of charges}

Charges can be generated by direct implantation with an electron beam. This is one of the ways electrets are formed [14]. By adjusting the energy of the incident electrons, the depth at which primary electron deposition is achieved can be controlled, a further advantage being that both the amount of charges and the position are controlled. For $30 \mathrm{keV}$ electron energy, Sessler reported (as measured) implantation depths of less than $10 \mu \mathrm{m}$ in films of FEP or Polyimide (PI) [58]. For PI, electron deposition goes beyond $150 \mu \mathrm{m}$ under $130 \mathrm{keV}$ electrons [59]. However, electron implantation goes with energy deposition, which is responsible for the so-called radiation induced conductivity [58]. Electron-holes pairs are created, which can be in far greater concentration than primary electrons, leading to defect creation to the polymer chains besides a modification of the transport properties in respect to virgin samples [60]. The same holds for proton irradiation [61]. Therefore, this way of charging cannot be considered as fully non-intrusive.

\subsection{4 lonizing radiation}

Ionizing radiations, being X-ray $[62,63,64]$ or $\gamma$-ray $[46,47,65,66]$, have been used in the context of charge trapping analysis mainly in conjunction with luminescence measurements, being isothermal luminescence or thermoluminescence. Here the effect of irradiation was not considered as a possible source of damage, even though doses as high as 1MGy were used for Polyethylene as an example [66]. However, high energy electron beam irradiation ( $\mathrm{few} \mathrm{MeV}$ ) is a way of radiation crosslinking of polymers $[67,68]$. With already a few tens of kGy, the melting point and infrared spectra of polyethylene are modified, showing that structural modification is at play $[69,70]$ while thermoplastic polyester films for example are stable up to the MGy range [71, 72]. Therefore, care should be taken with the interpretation of some of the data with radiation-induced charging. A difference with previous methods is that in principle samples are homogenously excited as ionizing particles loose only a fraction of their initial energy. 


\section{Techniques without stimulated detrapping}

\subsection{Surface potential decay}

Isothermal surface potential decay (SPD) is a well-known and robust technique to analyze the propensity of materials to relax charges. In the vast majority of experiments, charges are provided by corona discharge using a grid controlling the initial potential set at the surface of the material [36]. Other methods like contact charging [73], application of a DC voltage [74] or e-beam implantation are more seldom or dedicated to investigating the effect of a specific environment as with e-beam implantation [75]. Once the sample surface is charged to a given potential, the surface of the sample is scanned using a voltage probe and the decay of the potential is monitored as a function of time [36]. A variant of the technique is the mirror image method consisting in recording the image of the potential created by previously implanted charges in a scanning electron microscope [76]. The image is formed by the deviation of low energy electrons.

The surface potential decay kinetics is the only information accessible for tempting to access to trap characteristics. The information is macroscopic, i.e. there is no in-depth resolution of the charge. Therefore, a number of processes have to be hypothesized as reviewed by P. Molinié [36, 77]. First, three mechanisms are considered responsible for surface charge decay, i.e., bulk neutralization, which is in general the investigated process, gas neutralization by ions present in the environing air, and surface conduction due to the fact that experiments are usually realized with a limited surface area. The contributions from these different processes has been discussed recently by Zhang [78]. A second family of hypotheses is on the nature of charges: dipolar processes as well as charge motions can be at the origin of the SPD as observed for example for epoxy material [73]. Since only the discharge step is probed in SPD, the distinction between the two processes is not as obvious as for charging/discharging current measurements for example. Finally, the fate of charges is to be modeled: the deposited charges are primarily ions during the corona discharge and therefore there is some form of charge exchange from deposited ions to presumably electronic carriers, being electrons or holes, transported or being trapped in the sample bulk. Though, as mentioned previously, this charge exchange has been considered $[14,51]$ in most of the works this step is forgotten: it may be considered that the deposited charge is progressively 'injected' into the bulk and acts as a finite source of charge during the discharge process $[79,80]$ or the process is included in charge trapping on deep surface states [39].

Since its discovery by leda and coworkers [81], the crossover of the surface-potential decays of polymers has attracted considerable attention and has stimulated the development of various models, not necessarily involving trapping phenomena [75]. The crossover phenomenon corresponds to a faster decrease of the potential when the charging potential is higher. Models considering only the drift of charges with a possible field dependent mobility but without involving trapping / detrapping processes in general do not fit to experimental potential decay curves. Access to trap depth is given when detrapping processes are at play. The simplest way of analyzing SPD for trap depth estimation purpose is to consider that the decay is controlled by charge detrapping, i.e. the characteristic time constant for transport is shorter than the detrapping time. An image of the trap distribution is obtained with considering the following set of equations: 


$$
\begin{gathered}
t d V / d t \propto k_{b} T N\left(E_{t}(t)\right) \\
\text { and } E_{t}=k_{b} T \ln (v t)
\end{gathered}
$$

where $E_{t}$ is the trap depth, $k_{b}$ the Boltzmann's constant, $T$ the temperature, and $t$ the characteristic time of potential decay. Here $v$ is the attempt-to escape frequency in the frame of semiconductor physics model [33, 39, 82]. An equation of the form similar to Eq. (2) was derived in the frame of dispersive transport with a different expression for the attempt to escape frequency [36, 83, 84]. It corresponds to the general assumption that as time elapses, charges trapped in shallower states are progressively released and the charges emitted at a given time correspond to a preferential trap depth or energy demarcation [85].

Because the response is global, many assumptions are made when analyzing the surface potential decay rate. One of them is on the nature of decaying charge, dipolar vs. moving charges, as mentioned above. The refinement of models encompasses retrapping probability [85], consideration of different populations of traps, bipolar processes and possible charge recombination phenomena, various forms of 'injection' law, etc. With the advent of charge distribution measurement techniques, new information was brought and support from experiments could guide the theory. As an example, the crossover phenomenon was reconsidered with injection of charges from the ground electrode and bipolar charge transport and trapping [79]. Here the injection was taken with a Schottky law both for the charges at ground electrode and for those deposited by corona.

With the analysis succinctly presented above, in principle a continuous distribution of trap levels can be obtained. An underlying question is the presence of multiple processes controlling the decay, one related to the surface and one to the bulk of the material. In general, it is considered that charges trapped at the surface occupy deeper levels: it can be because the surface contains effectively chemical defects such as oxidized groups, etc. Actually modification of the surface may have strong effects on the charge stability $[86,87]$ and the discharge kinetics can be sensitive to the depth of deposited charges [88]. The alternative is that the appearance of deep traps reflects the 'injection' process at the surface.

An example of trap distribution obtained on low density polyethylene (LDPE) for positive and negative corona charging is shown in Figure 5 [39]. Here the potential decay has been fitted to a double exponential function, supposing that two populations of traps control the decay. The charges were supposed to decay independently. A OD model was implemented in which detrapping is the only process controlling SPD. The two populations would reflect physical and chemical traps into the material. Though nice results are produced, with distinct distributions, the analysis is biased by the hypothesis of double distribution each of them following an exponential decay for the SPD and by the fact that surface charge exchange processes are completely ignored. This kind of analysis has been repeated in recent years in different groups $[78,89,90,91]$ for different purposes. 


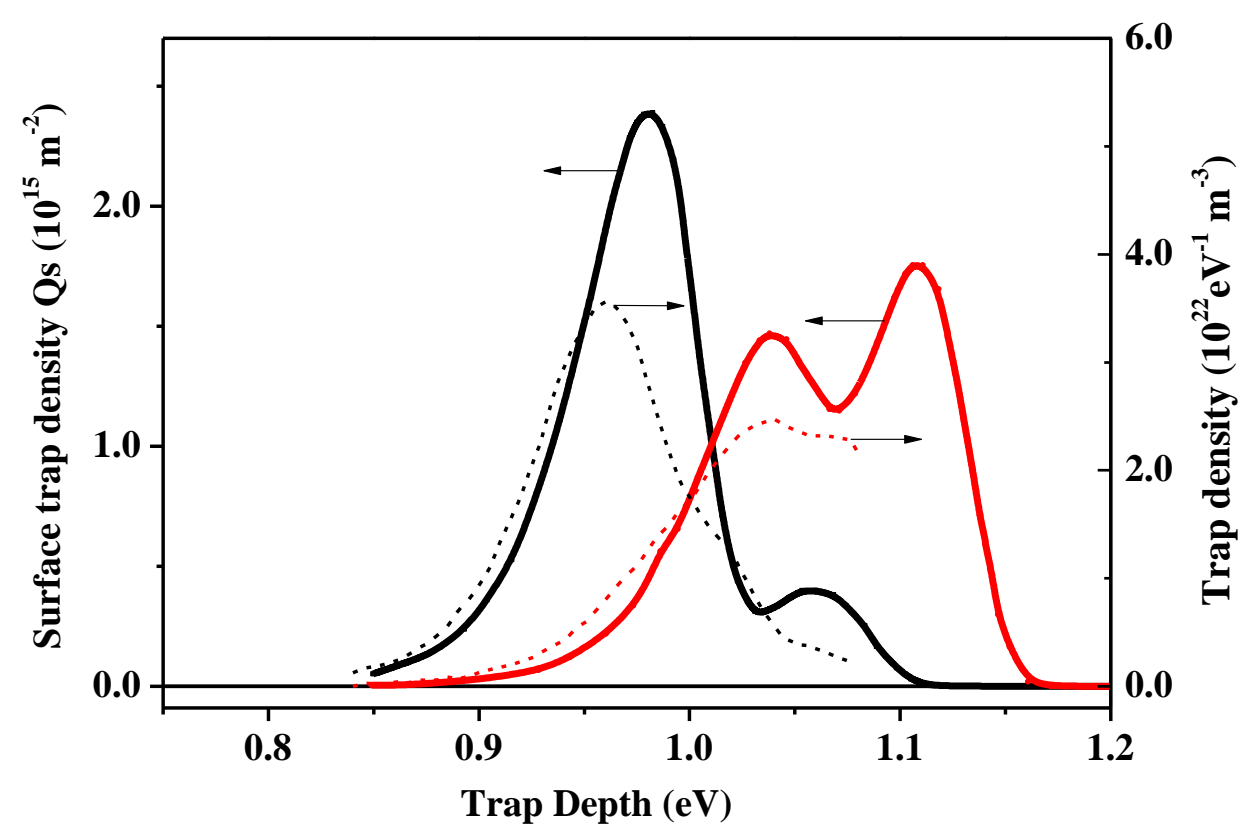

Figure 5. Bold lines: trap density distribution deduced from SPD analysis using OD-model in 25 $\mu \mathrm{m}$-thick LDPE after positive (red) or negative (black) corona charging, supposing a doubleexponential potential decay. Dashed lines: analysis of the same experimental data without double distribution hypothesis. Adapted from Li et al [39] and Shen et al [82].

Reprinted from [82], with the permission of AIP Publishing.

\subsection{Space charge decay}

The analysis presented above for SPD finds a parallel in the discharging step in space charge analysis. As it is necessary to get a good time resolution in space charge profiles acquisitions, and to record profiles repeatedly, essentially measurements by pulsed electroacoustic (PEA) method were implemented for that purpose. Data recording needs charging/discharging steps in space charge measurements, which does resort to current use of such technique. In respect to SPD presented above, the charging is achieved mainly by applying a DC field. Therefore, the charging time can be substantially longer than using corona contact, meaning that charges can drift during the charging time and occupy more the volume at the initiation of the discharge. Space charge measurements can be achieved too after charging with an electron beam $[92,93]$ and this may constitute an interesting alternative for the purpose of trap spectroscopy using low implantation depth of charges.

The first attempt to exploit space charge data for the purpose of extracting trap depths was that of Dissado et al [41], using results obtained by pressure wave propagation method applied in divergent field configuration with wire embedded in an epoxy matrix. Charges injected and trapped in the very vicinity of the wires are supposed to be thermally promoted directly from the trap level they occupy to an energy state. Then, they move almost instantaneously to the electrode and are extracted due to the high residual field. Besides, the number of trap states per unit volume per unit energy interval is a constant $N_{0}$. The decay is then entirely controlled by charge detrapping, and discharge models adapted from those initially developed for isothermal current discharge [33, 94] could be derived. With a typical decay curve of the total net stored charge $Q_{m}$ as plotted in Figure 6, quantities such as the minimum and maximum occupied trap levels, $\Delta_{\min }$ and $\Delta_{\max }$, the initial stored charge $Q_{M 0}$ or the rate of charge decay can be determined: 


$$
\begin{gathered}
Q_{M}(t=0)=Q_{M 0}=e N_{0}\left[\Delta_{\max }-\Delta_{\min }\right] \\
s=\frac{1}{Q_{M 0}} \frac{d Q_{M}(t)}{d \log (t)}=\frac{-k_{b} T}{\Delta_{\max }-\Delta_{\min }} \ln 10
\end{gathered}
$$

Here, s represents the charge resorption rate, expressed in \% per decade in time.

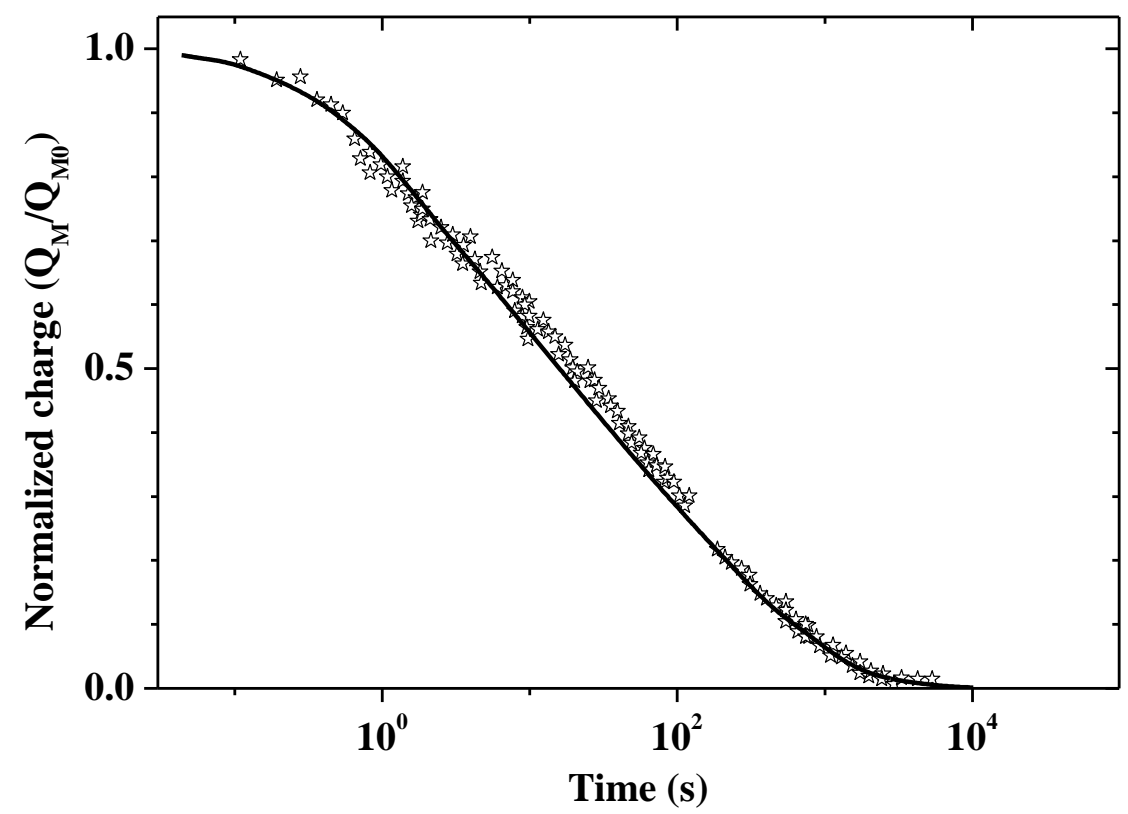

Figure 6. Experimental decay curve of total space charge amount as a function of time. From the analysis of the curve, minimum and maximum occupied trap state energy and discharge rate are deduced. Adapted from Dissado et al [41].

This initial analysis has been exploited for space charge measurements obtained by PEA for comparison purpose of different materials [95, 96]. Like for potential decay, the analysis of charge decay gives indication on the trap distribution in the range around $1 \mathrm{eV}$. This corresponds to detrapping times in the range minutes to hours.

A different approach of the charge decay was initiated by Mazzanti et al. [42]. By supposing that charges are localized near the electrodes, which can be actually verified since space charge distributions are handled, the discharging time-dependent mobility of charges could be determined:

$$
\mu(t)=\frac{\epsilon}{Q_{M}^{2}(t)} \frac{d Q_{M}(t)}{d t}
$$

where $\varepsilon$ is the dielectric permittivity of the material. In case where the distribution of initial charges is uniform, a factor 2 appears in the right hand term [97]. The conversion from a time-dependent mobility to a (occupied) trap depth distribution was achieved considering different hypotheses, as e.g. discrete or continuous trap distribution, or considering apparent trap controlled mobility, Eq. (5).

Using space charge decay method, the derived energy span for the trap depth is in the range around $1 \mathrm{eV}$ like in the previous analysis. The method was applied mainly to compare different materials using the same experimental procedures, as with XLPE with different formulations $[42,96,98]$. 
The technique is often employed in situation where charges are previously generated by applying a DC field, which may result in bipolar charging. Then, the absolute value of the charge is derived vs. time of depolarization. Tzimas et al [95] analyzed the decay of space charge amount in XLPE using two top hat distributions representing shallow and deep traps, and with attempting to differentiate the contribution from positive and negative space charges. It appeared that negative charge traps were in greater amount than positive ones (in unaged samples), and the energy depth was less. However, this kind of analysis could be implemented in situation where a single space charge domain is obtained after polarization by choosing the poling conditions. This should lead to more reliable results than those obtained by integrating positive and negative charge densities.

The isothermal methods presented above provide estimates in trap depths in the range $0.8 \mathrm{eV}$ to $1.1 \mathrm{eV}$ in LDPE as reviewed by Haque [99]. This reflects some form of consistency such presented. Table 2 represents and update and a correction to this previous review.

\begin{tabular}{lllll}
\hline \multicolumn{1}{|c}{ Material (form) } & $\begin{array}{c}\text { Energy } \\
\text { span (eV) }\end{array}$ & \multicolumn{1}{c}{$\begin{array}{c}\text { Method } / \\
\text { (Sign of charge) }\end{array}$} & Shape of Distribution & Reference \\
\hline LDPE & $0.85-1.03$ & PEA charge decay & 2 levels & Zhou et al [100] \\
\hline LDPE & $0.85-1.01$ & Integral charge decay & Discrete (2 main levels) & Haque et al [101] \\
\hline LDPE & $0.80-0.86$ & PEA charge decay & Discrete & $\begin{array}{l}\text { Montanari } \text { et al } \\
\text { [102] }\end{array}$ \\
\hline LDPE & $0.85-1.10$ & SPD (+) & Double distribution & Li et al [39] \\
\hline LDPE & $0.92-1.15$ & SPD (-) & Exponential & Haque et al [99] \\
\hline LDPE/FEP interface & $0.87-1.16$ & PEA charge decay & Top hat & Rogti et al [103] \\
\hline HDPE & $0.93-1.11$ & Integral charge decay & Montanari et al \\
\hline XLPE (various) & $0.83-1.07$ & PEA charge decay & Discrete & [102] \\
\hline XLPE (minicables) & $1.08-1.22$ & PEA charge decay & Discrete & Mazzanti et al [42] \\
\hline XLPE & $0.75-1.1$ & PEA charge decay (+) & Double Top hat & Van der Born et al \\
[98]
\end{tabular}

Table 2. Comparison of trap depth in LDPE and some other materials reported by different researchers. The sign of charges is indicated when it is reported in the results or when results resort to corona charging. 
The shape of the distribution in energy is not the same: about a top hat for Dissado et al [41], an exponential distribution for Haque [99] and a discrete [101, 102] or a two-level distribution [100] for others. Besides, the fact that the same span of energy is obtained can be in part due to the time scale of experiments which is roughly the same in all of the methods ( $\approx 1 \mathrm{~s}$ to a few hours). Especially, the low energy end of the distribution may depend on the switching time from charging to measurement. Considering other materials, for XLPE, we reported deeper energy spans, typically 1.0-1.2 eV [96] than those obtained by Mazzanti et al [42] but consistent with those of Van der Born et al [98]. All considered, traps appear deeper in XLPE compared to LDPE presumably because of traces of crosslinking by-products. For epoxy, reported data, at high fields, are in the range 0.68-0.94 eV [41].

\subsection{Charge Recombination-Induced Luminescence}

Charge recombination-induced luminescence-RIL-stands for the luminescence detected immediately after bipolar charging of a dielectric without any stimulation, i.e. isothermal luminescence without irradiation of any kind. Such effect is typical of situation where a critical degree of charging allows carriers of opposite signs to interact. Common situations where it arises encompass contact between an AC discharge and the surface of a film, charging of the sample under high field with the use of contacting metallic electrodes, or irradiating the sample with ionizing radiation generating pairs of charges. In all these examples, charges of both polarities are deposited (AC discharge), or injected (DC field), or created (ionizing radiation). The luminescence must be analyzed in time to separate those components that are due to long lived excited species formed during the charging step (typically $t<$ $1 \mathrm{~s})$, from the luminescence due to charge recombination which has a much slower kinetics being controlled by the recombination process (geminate recombination and drift recombination). We will illustrate these processes by taking two different examples being a contact between a cold AC plasma and a dielectric surface, and the charging of a sample under a high electric field, before discussing the contribution of this technique to the identification of trapping centers.

\subsubsection{Identification of the RIL component in plasma-induced luminescence}

Although the phenomenon of RIL is general and can be unraveled in most of the polymeric materials [104], we will illustrate the effect by taking the example of polypropylene [45]. Tiemblo et al [105] reported on Plasma-Induced Luminescence (PIL) measurements on polypropylene (PP) films after contact with a cold plasma of helium run at a frequency of $5.5 \mathrm{kHz}$ at atmospheric pressure. The experimental arrangement allows integral and wavelength resolved light measurements to be done in situ after discharge switch off. The kinetics of integral luminescence shown Figure 7 [45] is the sum of three components: the photoluminescence of PP, with time constant corresponding to the lifetime of triplet states excited by the UV of the discharge [54]; the chemiluminescence of PP [106] and the luminescence due to charge recombination with a typical kinetics, giving a total light decay in time of the form [21]:

$$
I(t)=I_{01} \exp \left(-t / \tau_{1}\right)+I_{02} \exp \left(-t / \tau_{2}\right)+I_{03}(1+\alpha t)^{-m}
$$

where $I$ is the luminescence amplitude, $I_{0 i}$ are pre-exponential factors, $t$ is the time elapsed from the end of the treatment, $\alpha$ and $m$ are adjustable coefficients and $\tau_{i}$ are time constants. The order of magnitude of $\alpha$ is $0.1 \mathrm{~s}^{-1}$ and $m \approx 1$. 


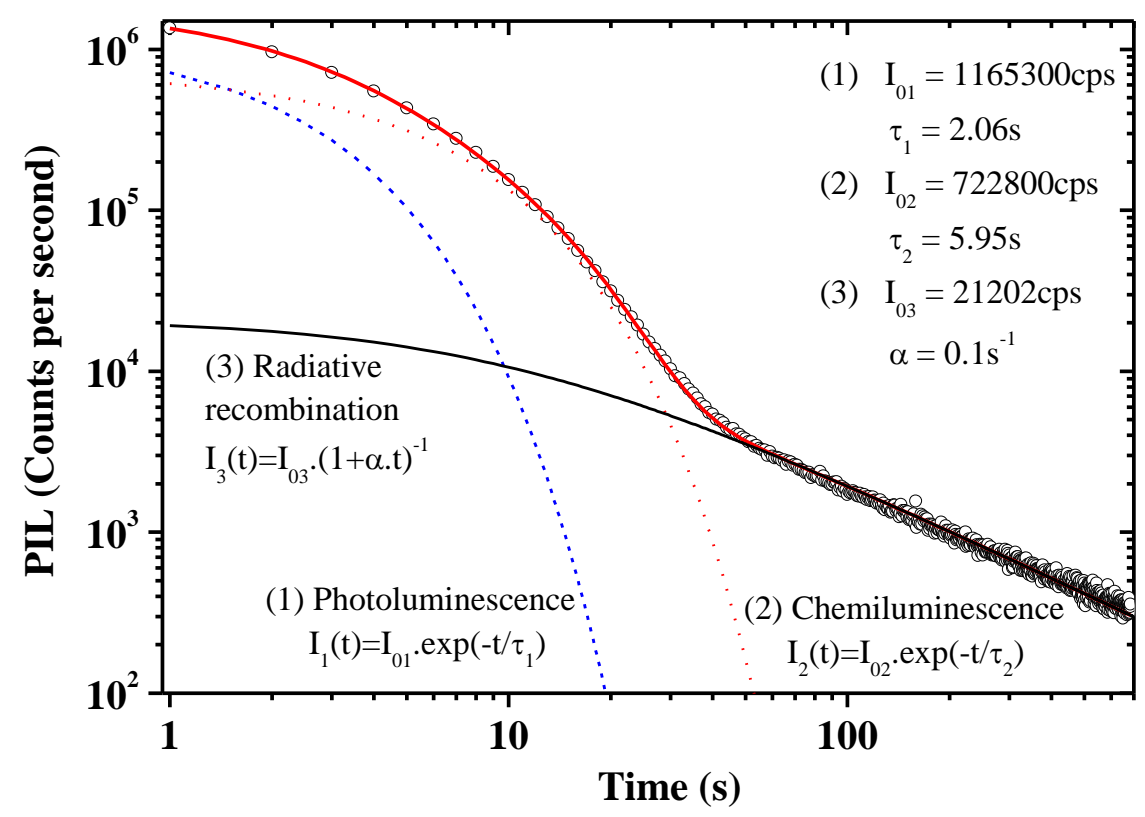

Figure 7. Time dependence of plasma-induced luminescence (PIL) following a 5 s treatment of a polypropylene surface at low temperature. Luminescence decay is the sum of three components as indicated. Circles: experimental data.

Reprinted from [45], with the permission of AIP Publishing.

The reciprocal time dependence of the light amplitude can be attributed to electron tunneling to positively charged luminescence centers $[47,107,108,109]$. Such kinetic analysis allows to define a time window where recombination is the sole contribution and therefore to isolate the emission spectrum of the process as shown in Figure 8. Here the emission characteristic of radiative charge recombination peaks at $510 \mathrm{~nm}$. Its origin is discussed later on.

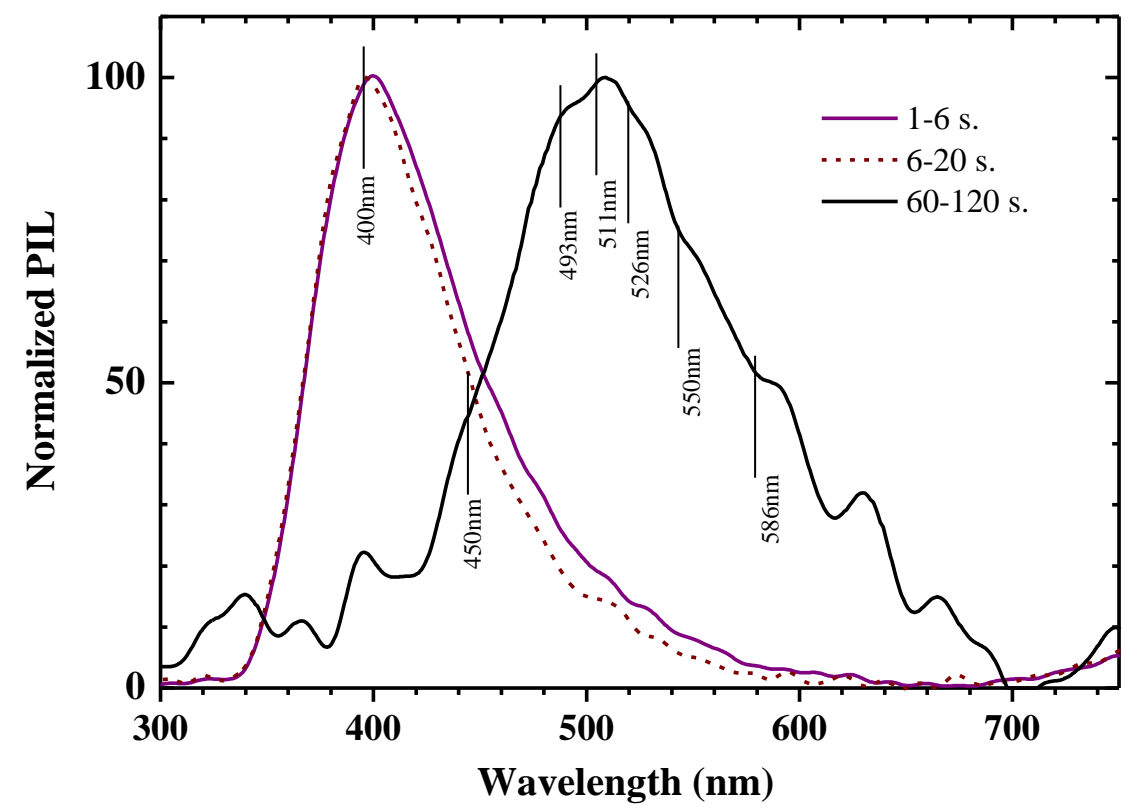

Figure 8. Time dependence of plasma induced luminescence spectra for PP at LNT. Spectra are normalized to the emission maximum. Integration time windows are given in the caption. Wavelengths indicated on the spectra are real emission maxima or shoulders. Other structures can be due to data smoothing procedure. 


\subsubsection{RIL following DC charging}

The deposition of charges of the two polarities at the surface (or in the volume using ionizing radiation) constitutes an efficient way of revealing charge recombination processes. Interesting is the case where charges are generated by the application of a DC field: delayed electro-luminescence was observed. Although the phenomenon is observed in different polymeric materials $[110,111]$ it will be illustrated with experiments performed on polyethylene naphthalate -PEN as this material is highly luminescent when excited due to the aromatic ring structure in the repeat unit $[112,113]$. This renders detection of the luminescence much easier and therefore allows a much deeper insight.

Under DC stress, charges of both polarities are generated through charge injection at both electrodes $[114,115]$. Delayed luminescence is shown in Figure 9 [116]. Note that the experiment is realized using a high voltage switch in order to disconnect the sample form the source. When the sample is grounded, the delayed luminescence is observed but its decay is much faster [114, 117]. This situation is much more difficult to handle, because the space charge formed during field application can generate a much higher internal field when the electrode potential is set to zero. In fact, this field can be high enough to induce sample breakdown [118].

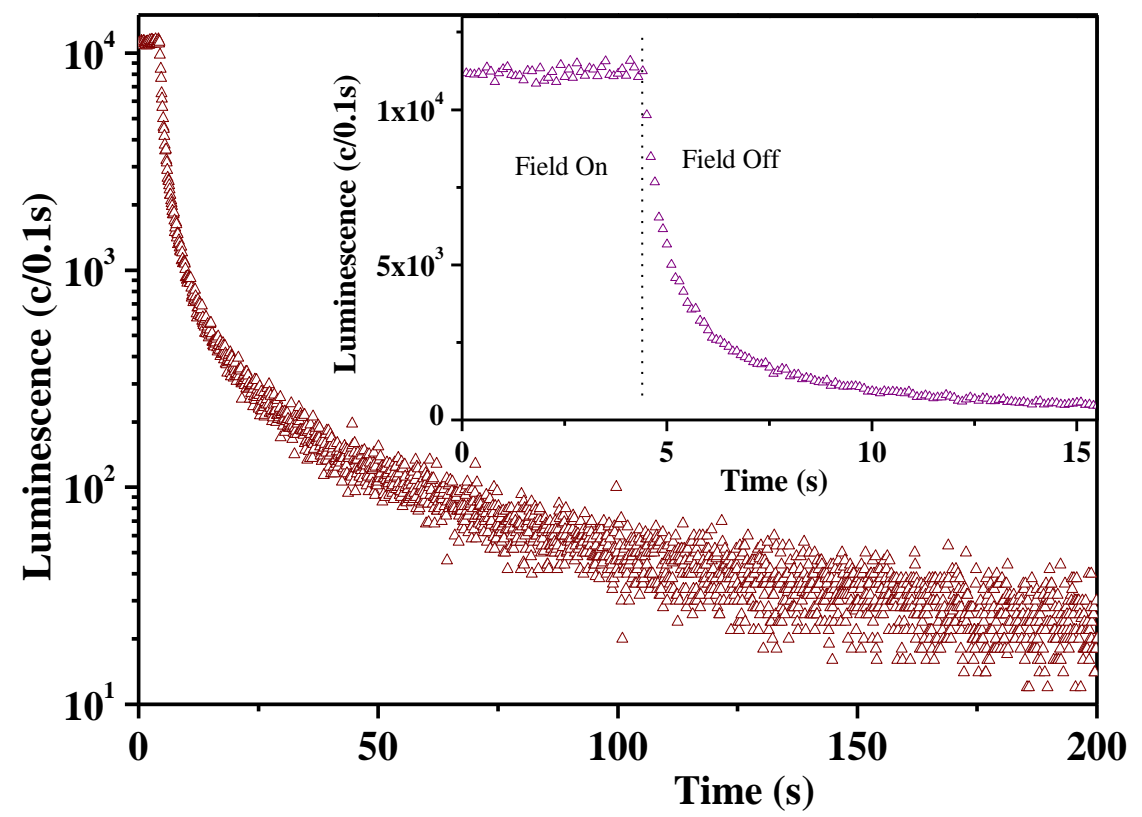

Figure 9. Time evolution of the luminescence observed in PEN during and after application of a DC field of $310 \mathrm{kV} / \mathrm{mm}$ at room temperature.

Reproduced from [116]. @ IOP Publishing Ltd. All rights reserved.

Two main effects could contribute to the light decay if we neglect the fluorescence lifetime which is much too short $(<<1 \mu \mathrm{s})$ to play any role in the considered time scale. One is due to the phosphorescence lifetime of the PEN molecules that were excited during field application, according to:

$$
{ }^{3} \mathrm{PEN}^{*} \stackrel{{ }^{3} \mathrm{k}_{1}}{\longrightarrow} \mathrm{PEN}+{ }^{3} \mathrm{hv}
$$

Where $h v$ is the radiated light ( $h$ is the Planck's constant and $v$ the frequency). 
The other is due to charge recombination on luminescence centers, with a decay controlled by the kinetic of the recombination process and the lifetime of the excited states, according to:

$$
\mathrm{PEN}^{+}+\mathrm{e}^{-} \text {(trapped) } \stackrel{\mathrm{k}_{2}}{\longrightarrow}{ }^{1,3} \mathrm{PEN}^{*}
$$

where the rate constant $k_{2}$ is characteristic of the recombination mechanism. Then,

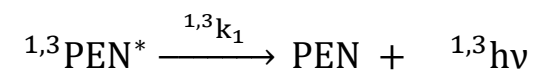

with a rate constant ${ }^{1} k_{1}$ and ${ }^{3} k_{1}$ for fluorescence and phosphorescence transitions respectively.

We have checked that the phosphorescence lifetime, of the order of $20 \mathrm{~ms}$, cannot account for the light decay that is therefore controlled by charge recombination. The analysis of the decay was achieved considering a distribution of recombining electron-hole pair separation distances and supposing that geminate recombination dominates. A mean distance of about $1.8 \mathrm{~nm}$ has been obtained [116]. Being the sole mechanism at play, emission spectra could be acquired along the decay.

\section{Techniques with stimulated detrapping}

With using thermostimulated relaxation of a material, the experimental time can be considerably shortened and a broader number of relaxation modes explored in a single spectrum compared to isothermal decay. In addition, the possibility to 'freeze' a charge state by cooling may avoid the fast detrapping from shallowest levels that occurs just after charging in isothermal conditions.

\subsection{Thermally stimulated currents}

Though the first reports on thermally stimulated discharge current (TSDC) date back to 1936, its systematic use and kinetic analysis appeared in 1966 [119]. TSDC method has been very popular in the years 70 to 90 , to probe dipolar processes as well as detrapping of charges [120]. Van Turnhout -who provided a considerable contribution to the topic - proposed a very extensive review of the method applied to investigate charge stability in electrets [49], and only a brief outlook will be given here.

The principle of the method consists in charging the material at high temperature (e.g. by application of a dc field on a metallized sample), followed by cooling to low temperature with the field still maintained. Next, it is short-circuited and reheated at a linear rate of a few ${ }^{\circ} \mathrm{C} / \mathrm{min}$, the discharge current generated being measured with an electrometer, and recorded as a function of temperature. The pre-charging of materials is done using one of the methods described previously. However, as dealing with current measurements, it must be ensured that the charges move over significant distance relative to the electrodes to collect a measurable current. If charges are trapped the very surface and collected on the adjacent electrode, then the current is virtually null. This sensitivity to the position of the charges is an issue for quantification purpose. One way to overcome the problem consists in interposing an air gap between the non-metallized surface of the material and the measuring electrode [121], but this limits the characterization to non-metallized films and may be more difficult to implement than with short circuited electrodes. The heating can be made with a field being applied; with as objective to drive the charges in a controlled direction, the method is called in 
principle TSC. Vanderschuren discussed the differences in the results of TSC and TSDC in case of dipolar processes [122].

The simplest expression of the TSDC glow curve is that corresponding to relaxing dipoles from a homogeneous polarization of a dielectric with shorted electrodes.

$$
J(T)=\frac{P_{0}}{\tau_{0}} \exp -\frac{E_{a}}{k T} \times \exp \left[-\frac{1}{\delta \tau_{0}} \int_{T_{0}}^{T} \exp -\frac{E_{a}}{k T^{\prime}} d T^{\prime}\right]
$$

where $J$ is the current density, $P$ the polarization $\left(P_{0}\right.$ the initial polarization, in $\left.\mathrm{C} / \mathrm{m}^{2}\right), \delta$ is the heating rate. The relaxation time $\tau(T)$ has a Arrhenius time dependence:

$$
\tau(T)=\tau_{0} \exp \frac{E_{a}}{k T}
$$

where $\tau_{o}$ is the frequency factor, $E_{a}$ the activation energy and $k$ the Boltzmann's constant. In case of TSDC resulting from moving charges, with or without detrapping, the situation is far more complicated. The discharge current is obviously dependent on the initial charging state of the material. It seems that only in very restricted situations the above analysis can be valid: that of an initial charge cloud close to the surface without contacting it and discharge controlled by the mobility of charges under the effect of their own field, up to the time internal charges reach one of the electrodes. An equivalent situation is that of detrapping with fast retrapping [49]. For any other distribution and situation, there is no tractable equation. There are limit cases of charges adjacent to one electrode and evacuated at that electrode and the one with homogeneous distribution of charges that provide no external current. We should mention the use of the Thermally Stimulated Conductivity -TSC- method that consists, most often, in sensitizing the material with ionizing radiation and measuring the current under field. There is a priori no need to consider non-homogeneous processes along the thickness, which may make the analysis easier. However, ionizing radiations are highly intrusive [123] and detected traps may be not relevant only to the initial material state, particularly in polymers that are sensitive to irradiation.

Chiefly, the methods for the determination of the thermal activation energy or the frequency factor can be divided into three categories: those making use of heating rate variations, those based on geometrical approximations of the glow peak and among various others, the Garlick's and Gibson's initial rise method [124]. According to the latter, the initial rise of a glow peak follows an Arrhenius equation, which slope gives the thermal activation energy [123].

The first method is based on the following relation between heating rate and glow curve parameters:

$$
J_{m}=\frac{\delta \cdot E_{a} P_{m}}{k T_{m}^{2}}
$$

where $J_{m}, P_{m}$ and $T_{m}$ are respectively the peak current, the area under the glow curve and the peak temperature. It is not a very precise method as the peak position is not so much sensitive to the heating rate, and a very good control of temperature under different heating rates is necessary. The second one supposes that the hypotheses behind the model are fulfilled during all the depolarization process. In the case of Debye peak with a single relaxation time, the time dependence of the relaxation time is obtained from: 


$$
\tau(T)=\frac{P(T)}{J(T)}=\frac{1}{\delta} \frac{\int_{T}^{T f} J\left(T^{\prime}\right) d T^{\prime}}{J(T)}
$$

where $P(T)$ is the remaining polarization at a given temperature. Then the activation energy can be obtained from the regression line on $\tau\left(T^{-1}\right)$ according to Eq. (11). Figure 10 shows an example of peak analyzed using this method [125]. The pure Debye-like peak obtained with the fitted activation parameters is represented as solid line.

Finally, the initial rise method provides the activation energy from the low temperature side of the glow curve, when nearly all the polarization remains in the dielectric, cf. second term in Eq. (10). This last method is more robust as it considers only the initial rise of the glow curve.

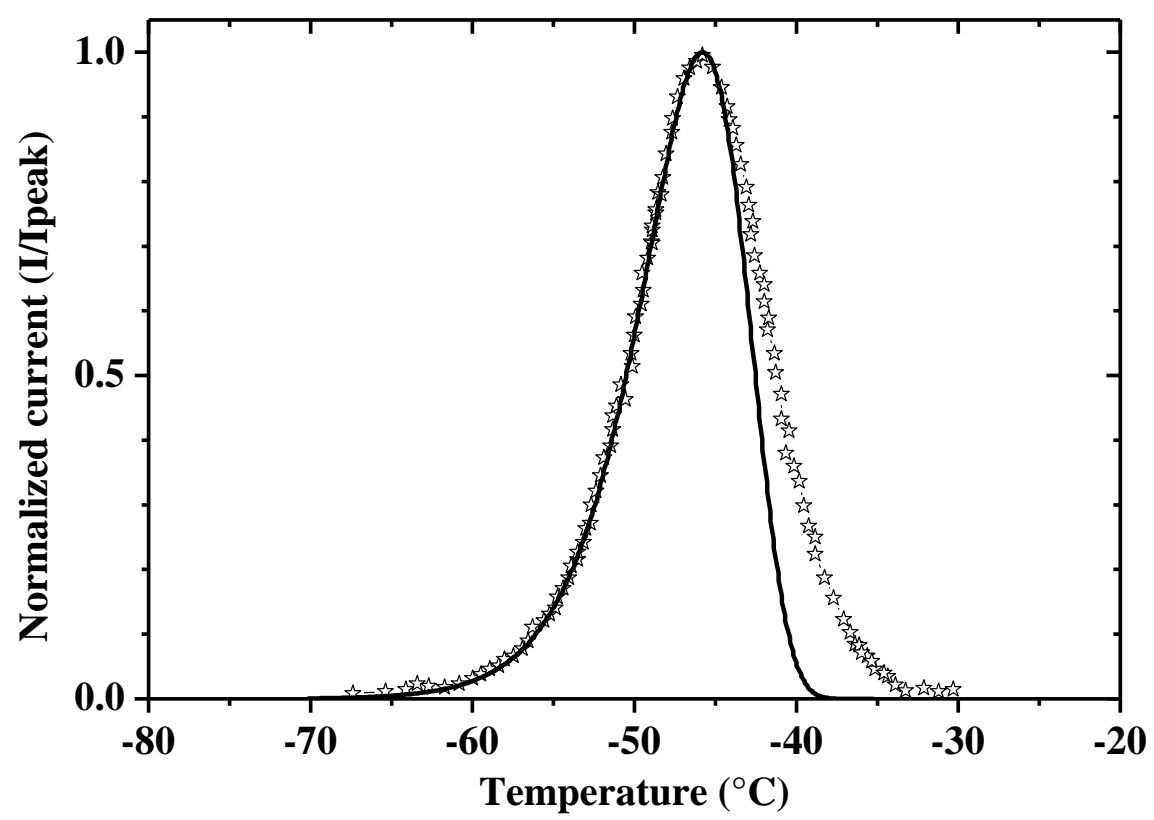

Figure 10. Example of experimental fractional polarization TSDC peak (solid line) analyzed as a Debye-like peak using the rising of the peak and Eq. (13) and computed using Eq. (10). $E_{a}=1.34 \mathrm{eV} ; T_{m}=-45.8^{\circ} \mathrm{C}$. Reproduced from [125]. @ IOP Publishing Ltd. All rights reserved.

If the frequency factor were known, the activation energy would be deduced easily from the relation between peak temperature, and activation parameters:

$$
\tau_{0}=\frac{k \cdot T_{m}^{2}}{\delta \cdot E_{a}} \exp -\frac{E_{a}}{k \cdot T_{m}}
$$

Behind this analysis, there is one fundamental question related to the pre-exponential factor that is supposed either constant or specific to the process involved. A resolution of the glow curve following Eq. (13) may lead to values for the frequency factor $\tau_{o}$ that are extremely small, with a vexing question about its physical meaning. It has been proposed that $\tau_{o}$ which in the frame of semiconductor physics is a phonon frequency or a vibrational frequency of the lattice, is not reduced to $k_{b} T / h$ in ArrheniusEyring equation, but contains an entropy term that accounts for the multi-excitation steps necessary to overcome a potential barrier $[126,127]$. The higher the barrier to overcome, the larger the number 
of intermediate steps, and hence potentially the higher the entropy term, leading to a relation between the energy and entropy terms of the kinetics. This very general approach holds also for transport processes.

The passage from a single relaxation to distributed relaxation processes is a difficulty in itself. Many attempts have been proposed to resolve complex TSDC spectra into a distribution in relaxation times, which can be in the activation energy, in the pre-exponential, or both [49, 122, 128]. Experimental variants of the TSDC method exist to produce discrete distributions of processes like fractional polarization -i.e. polarization in a narrow temperature window, or by step-by-step decomposition of glow curves by first fitting the most prominent peak, subtracting it from the glow curve and then continuing this procedure for the remaining side peaks until a satisfactory fit is achieved [129]. We shall come back to this aspect in the discussion section. In the analysis of charge detrapping, this difficulty combines with that linked to the incertitude on the position of the charges giving rise to a depolarization current.

In the implementation of TSDC for trap spectroscopy purpose, many further difficulties can be met. First, on a technical point of view, the heating rate must be perfectly linear, otherwise artefacts can be produced as the recorded current is proportional to the heating rate. There may be coexistence of different processes to the TSDC, one of them being the relaxation of orientation polarization that produces a current of opposite sign to the charging current. A number of criteria have been proposed to distinguish discharge due to dipoles from carriers detrapping, as the linearity with applied voltage, the impact of the nature of electrodes or the impact of the polarization temperature. Still with the coexistence of processes, the discharge can be not necessarily due to detrapping but to ohmic conductivity leading to compensation of the stored charge.

At this stage, the resolution of the problem of reconstructing TSDC spectra should be done using fluid models of transport, that provide without approximation the current and charge redistribution corresponding to a set of hypothesized physical processes [130] with however the difficulty to identify model parameters likely to describe the full process.

There are many examples of application of TSDC in the literature for trapping parameters estimation. For example, Kojima et al [22] observed strong polarity effects on part of the TSDC features in Poly(ethylene terephthalate) (PET) and could in this way separate dipolar processes from detrapping in the response. They obtained a trap depth of the order of $0.65 \mathrm{eV}$. Interestingly, the activation energy for dipole relaxation almost coincided with that for electronic carriers detrapping leading to consider that thermal detrapping processes are strongly affected by molecular motion.

Rytöluoto et al [131] reported recently on TSDC from Polypropylene capacitors. Trap energies in the range from 0.3 to $1.3 \mathrm{eV}$ were obtained by multi-peaks fitting, with positive or negative contributions to the glow peaks.

TSDC from polyethylene materials was discussed mainly on a qualitative basis regarding the sign of the TSDC current and the comparison of corona vs. DC field charging [132]. The initial rise method was used in TSC of X-ray irradiated HDPE to probe oxidation effects on the material. Trap depths in the range 1 to $1.5 \mathrm{eV}$ were obtained above room temperature with the method, and tentatively attributed to detrapping from different structural regions [133]. 


\subsection{Thermally Stimulated Luminescence}

Thermally Stimulated Luminescence- often called Thermoluminescence (TL) consists in analyzing the light produced by a material during a linear heating rate once sensitized. In the core of the literature about the topics, particularly over the recent years, research on TL is dominated by application to scintillators in which trap depth as well as quantitative aspects are important features. In these cases, the sensitization is made using high-energy ionizing radiations. Materials for such application are usually inorganic crystals, organic crystals or doped organic materials, with in recent years the proposition of using common plastics as Poly(ethylene naphthalate) [113, 134]. There are comparatively few research reports dealing with TL following DC field application or more generally net charge implantation. Nishitani et al performed simultaneously TSDC and TL measurements after applying a high DC field to PE samples at low temperature (90K) [135].

TL can be viewed as a complementary method to $\operatorname{TSC}$ or $\operatorname{TSDC}[25,123]$. Wintle pointed out the inconsistency between results of the two techniques [136, 137], inconsistency that can be explained in part by the fact that the latter probes both dipole and charge relaxation whereas the first is sensitive mainly to charge relaxation, and more precisely charge recombination. Therefore, looking at the details, the techniques can be viewed as complementary more than contradictory.

With considering simple hypotheses, a relatively tractable equation can be derived for the TL glow curve [138]. The hypotheses taken are provided in Figure 11 with detrapping electrons, from a single detrapping level, recombining with trapped holes.
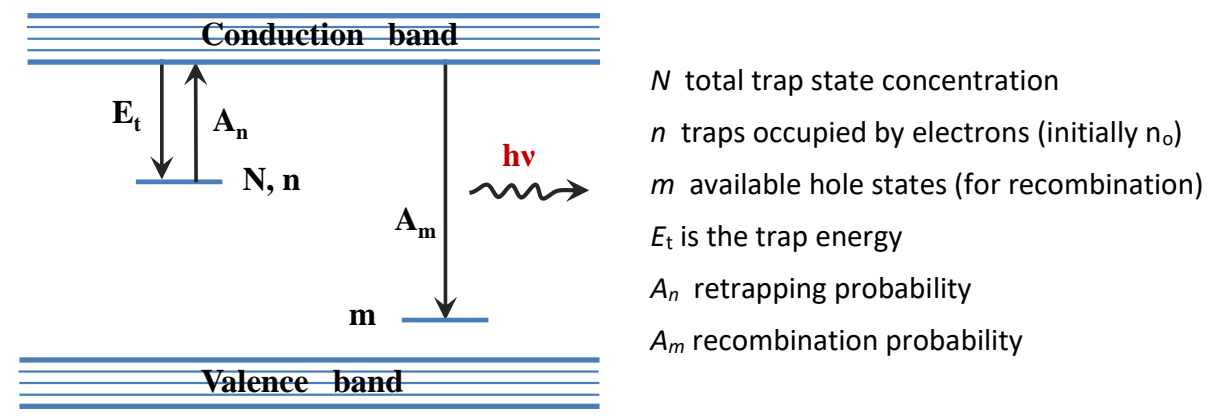

Figure 11. Scheme of processes involved in thermoluminescence in its most simplified assertion. Reproduced from [138]. (c) IOP Publishing Ltd. All rights reserved.

It is considered that the rate at which electrons escape from the traps is roughly governed by the vibrational frequency $S$ at which electrons interact with the lattice within the trap and the trap depth $E_{t}$. The overall escape rate is proportional to $S \exp -E_{t} / k T$. We suppose that there are more accessible holes than detrapped electrons ( $m>>n)$ and a strong recombination probability $\left(A_{m}>>A_{n}\right)$, then the glow TL curve is described by:

$$
T L(T)=n_{0} S \exp -\frac{E_{t}}{k T} \times \exp \left[-\frac{S}{\delta} \int_{T_{0}}^{T} \exp -\frac{E_{t}}{k T^{\prime}} d T^{\prime}\right]
$$

where $\delta$ is the heating rate. This equation exactly corresponds to the one for Debye peaks for dipoles and it reflects first order kinetics, Eq. (10). When fast retrapping is effective and traps are far from saturation, then second order kinetics holds, and analytical solutions can be found with some 
hypotheses. In the general cases, only general order kinetics can be applied. Eq. (15) then becomes [139]:

$$
T L(T)=n_{0} S \exp -\frac{E_{t}}{k T} \times\left[1+(b-1) \frac{S}{\delta} \int_{T_{0}}^{T} \exp -\frac{E_{t}}{k T^{\prime}} d T^{\prime}\right]^{\frac{-b}{b-1}}
$$

where $1<b \leq 2$ is the kinetic order parameter ( $b=2$ for second order kinetics).

In practice and in the context of TL applied to dosimetry, first order kinetics satisfactory describes TL glow curves; however, for different reasons the fitting parameters do not have the simple meaning of trap depth and escape frequency [140].

The different methods of analysis of TL curves have been reviewed by Kirsch [139] whereas Kivits and Hagebeuk [141] discussed about the accuracy of the approximations made. In principle models for TL belong to the same families as those for TSDC curves, with an extra parameter that is the kinetic order. The initial rise method provides activation energy whatever the kinetic order, but does not provide information on the order. The information on the order is brought by the width of the glow curve. Linearization or curve-fitting methods are used to extract kinetic parameters. A number of artefacts as too low or two high activation energies have been discussed by Chen [142]. They correspond grossly to the case where first or second order kinetics seems in operation, but the detailed mechanisms are more complex.

Isothermal luminescence decay, like presented in Section 4.3, can be a way to check the order of the kinetics [143]. A recent work on long-lived phosphors points to the equivalence in the mechanisms controlling isothermal luminescence decay and TL [144]. In general, the analysis of overlapping detrapping processes is more precise through TS techniques compared to isothermal ones [139]. Using the spectral analysis and recording distinctly the decay in different spectral windows can provide more precise resolution [145].

In principle TL more closely reveals detrapping events than TSC/TSDC, as transport is not involved and other thermally activated processes like dipolar relaxation do not produce light while they produce a current. However, recombination may occur without excitation to the extended states. The charge transfer can occur via tunneling between spatially correlated electron and hole trapping centers within defect complexes, corresponding to geminate pair recombination or across randomly distributed centers; this latter type of recombination is known as localized recombination [146, 147]. The latter model involves three states, namely the ground and the excited state of the donor, and an undefined state of the acceptor center; recombination occurs via the excited state on stimulation by heat or light. The power law decay behavior observed for ground state tunneling has generally been explained by a distribution of donor-acceptor ( $d-a)$ separations (tunneling distances). It can be anticipated that the optical or thermal energy involved in the recombination process is less than the trap depth. Jain et al [147] computed TL curves considering a distribution of tunneling separation distances, such distribution in separation being previously used to explain the power law decay of isothermal luminescence $[108,109]$. The glow curves have much slower decay than the first order kinetics TL curves. However, the story does not tell about the apparent activation energy. 
As mentioned previously, the analyses of TL and TSC have been combined in several studies to provide a more complete view of the processes at play. The fact that peaks appear in the same temperature range is not a proof of the same origin in the processes. Markiewicz et al [50] realized simultaneous measurements of TL and TSC on LDPE, using optical bleaching and treating materials in different conditions such as forced oxidation (see Figure 12) or attack by fuming nitric acid. As shown in Figure 12 , oxidation has opposite effects on peak magnitude. It was concluded that molecular motions could drive geminate pair recombination in TL while TSC was not due to charge detrapping but to dipoles motion. Suzuoki [148] reported on the decrease of TL intensity after oxidation of high density PE. They ascribed the feature to deepening of charge traps after oxidation. Optical bleaching consisting in redistributing charges in traps by using light indeed showed that higher energy is necessary (3.0 vs. $1.9 \mathrm{eV}$ ) to induce a decrease of the TL activity.

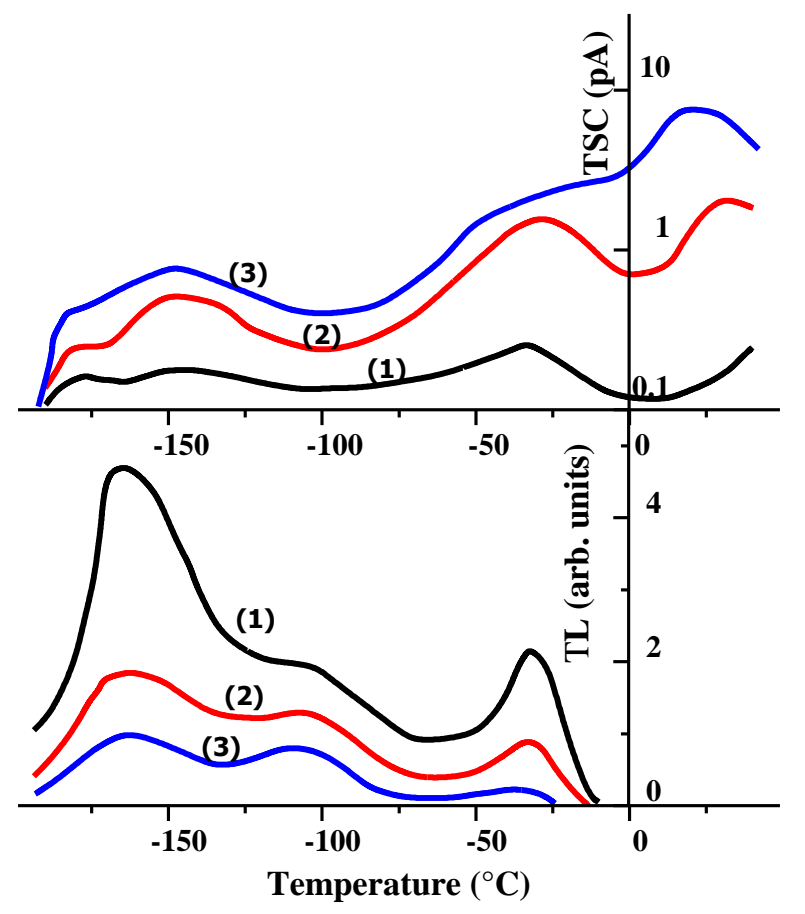

Figure 12. Simultaneous measurements of TSC (top) and TL (bottom) on Low Density Polyethylene after exposure to $\mathrm{O}_{2} / \mathrm{O}_{3}$ mixture at $40^{\circ} \mathrm{C}$ for (1): $0 \mathrm{~h},(2): 25 \mathrm{~h}$; (3): $150 \mathrm{~h}$. Reprinted by permission from Springer Nature Customer Service Centre GmbH: [Springer] [Thermal Analysis] [50] (1991).

A noticeable difference between TL and TSC is the fact that TL is more effective at low temperature, presumably for the higher probability of radiative de-excitation than at high temperature, while TSC should not in principle follow temperature-dependent efficiency.

\subsection{Photostimulated Discharge}

Photo-stimulated current spectroscopy (PSC), also called photo-stimulated discharge method (PSD), consists in charge release from their traps induced by irradiation of the sample with monochromatic light. This technique can be seen as the optical equivalent of the TSCD method. The first papers dealing with PSD theory and measurements on insulators and semiconductors date back to the early 1970 
$[149,150]$, and a small number of papers have been published since then, which implies that PSD is a relatively confidential technique compared to TSDC.

The first step of the experiment consists in traps filling with methods as charge injection from the electrodes under high electric field or irradiation by corona discharge or electron beam described previously. The trapping level can also be filled using photo-injection of electrons from the lighted electrode into the material bulk [150] with application of an appropriated electric field. Photoemission and transport are then accelerated by the applied external field and a fraction of the electrons is trapped in deep stable traps at the energy $E_{T}$ (Figure 13a). It can be also necessary to maintain low temperature to avoid thermal emptying of the traps.

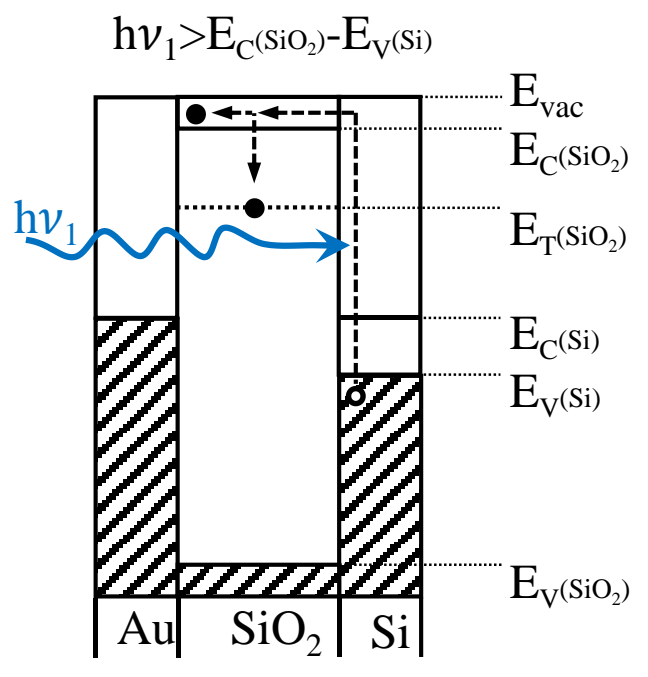

(a) Photoinjection $\mathrm{h} v_{2}>\mathrm{E}_{\mathrm{C}\left(\mathrm{SiO}_{2}\right)}-\mathrm{E}_{\mathrm{T}}\left(\mathrm{SiO}_{2}\right)$

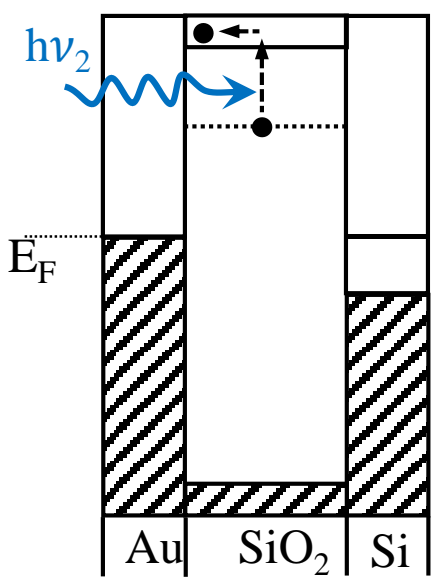

(b) Photodepopulation

Figure 13. Idealized energy band diagram for $\mathrm{Au} / \mathrm{SiO}_{2} / \mathrm{Si}$ structure.

Reprinted from [150], Copyright (1972), with permission from Elsevier.

The second step consists in the excitation of the charges trapped in defect centers to the conduction band. The photons energy needs to be higher than the energy $E_{C}-E_{T}$ (Figure $13 \mathrm{~b}$ ). The external current is recorded as a function of time for a fixed wavelength (Time resolved photo-depopulation) or during wavelength scanning at a constant rate (Spectrally resolved photo-depopulation). In a first approximation the trap depth corresponds to the long-wavelength cut-off of the PSD peak for wideband materials, while for narrow-band materials the peak of the spectrum corresponds closely to the trap depth [151].

Typical PSD spectra obtained on a polyimide (PI) film charged under electron beam irradiation [28] are shown on Figure 14. Two dominant trap depths corresponding to a photon energy of $2.67 \mathrm{eV}(465 \mathrm{~nm})$ and $3.20 \mathrm{eV}(390 \mathrm{~nm})$ are observed.

The difficulties in the interpretation of the PSD data (as well as to compare the results obtained from different authors) lie in the large number of factors that can affect the PSD current:

\subsubsection{The experimental parameters linked to the PSD setup}

An arc lamp associated with a monochromator is often used to produce quasi-monochromatic light. The spectral energy distribution of the light is taken into account by normalization of the recorded PSD current [26], especially when working in the near UV-range. Filtering of the infrared part of the 
irradiation spectrum avoids heating of the sample. Thermal de-trapping can then be considered as negligible when PSD is performed. The bandwidth of the produced quasi-monochromatic light, which is directly related to the spectral resolution of the PSD method, is of a few nanometers (from 4 to 20 $\mathrm{nm}$ ) in most of the published papers. Finally the scanning rate used for spectrally resolved photodepopulation (continuous scanning from low to high energy) should be small enough to allow the traps to be completely emptied for each energy level, otherwise the PSD currents can be overestimated, due to the detrapping of the shallower traps under the irradiation of higher energy photons [152].

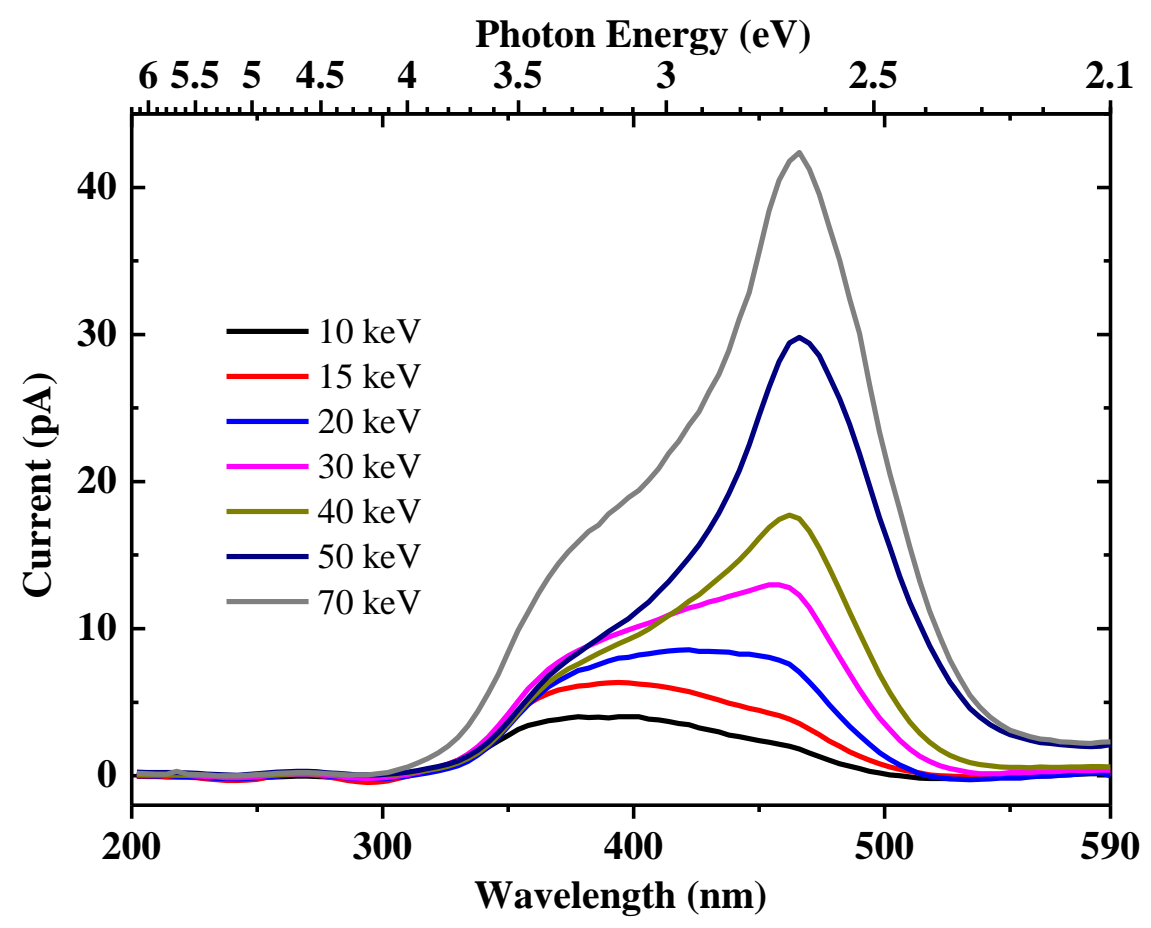

Figure 14. PSD spectra of polyimide charged under electron beam irradiation. Reproduced from permission of Eur. Phys. Lett. [28].

\subsubsection{The experimental parameters linked to the sample and electrodes}

The metal nature of the electrodes, through the work function, directly influences the PSD response. Ma et al [153], studying the photo-stimulated discharge spectrum of polypropylene films, showed that the position of the peak is shifted to the longer wavelengths when Au (work function of $5.1 \mathrm{eV}$ ) is replaced by $\mathrm{Al}$ (work function of $4.28 \mathrm{eV}$ ) for the electrode facing to the light irradiation. The thickness and geometry of electrodes are also important parameters. To ensure the light penetrating into the material under study, the transmittance of the illuminated electrode has to be taken into account. For example a $23 \mathrm{~nm}$-thick gold layer shows transmittance of approximately $40 \%$ [154]. However, the transmittance of a metal layer is depending on the light wavelength, with an important decrease in the near-UV range. In order to improve the signal-to-noise ratio in PSD measurements, transparent and conductive material like Indium Tin Oxide (ITO) could be used as illuminated electrode [155]. Another way is to use a fingered or structured electrode facing to the irradiation light. The PSD signal is higher because of higher light penetration within the material bulk. On the other hand, one of the indirect effects of using such fingered electrode is that electric field can be enhanced locally due to edge effects, and as a consequence charge injection and trapping at these "hot points" can be 
substantially increased [153]. Finally, the interpretation of PSD signal can be difficult in the case of nonuniform spatial and energy distribution of the traps, because the penetrating depth of the incident light into the material depends upon the wavelength. For example, the penetrating depth is estimated at about $7.2 \mu \mathrm{m}$ at the wavelength $470 \mathrm{~nm}$ and $3.3 \mu \mathrm{m}$ at $370 \mathrm{~nm}$ in polyimide [154].

\subsubsection{The additional physical processes that can be at play}

When dealing with photoconduction, optical excitation can take various forms, ranging, besides photodetrapping of carriers, from photo-injection of carriers at the electrode-dielectric interface, excitation of $\pi$ electrons in conjugated groups with bound electron-hole pairs creation, which dissociation can be field-aided [156, 157, 158, 159]. Even though charge release from traps is the main mechanism behind the PSD current, it appears that some of these processes combining optical excitation and field application can be at play also in photo-discharge experiments [48].

For example Mellinger et al [26] studied the photo-stimulated discharge in some polymer electrets (polytetrafluoroethylene (PTFE), polyethylene terephthalate (PET) and polypropylene). They concluded that the external photo-effect must be taken into account only when using pressed-on electrodes at short wavelengths (under $260 \mathrm{~nm}$ ). Furthermore, the authors assume that the interaction of excitons and trapped charges leads to a change in the charge distribution and further annihilation of trapped charge. In a recent study of the photocurrent spectra of polypropylene films under different electric and geometrical conditions, Ma et al [160] showed that when a local strong positive electric field exists near the finger electrode photoemission from finger electrode is observed, and partially contribute to the PSD peak located at $270 \mathrm{~nm}$ [160].

The recent improvement of the PSD method concerns the development of new methodologies as the step scanning method and the combined calibration-scanning method [152], or the use of optical parametric oscillator (OPO) laser as irradiation source that allows to reach higher energy resolution, at around $0.01 \mathrm{eV}$ [48]. The step scanning method consists in irradiating the sample by successive wavelength step (i.e. step-by-step mode from low to high energy), step duration being equal to the time necessary for the photocurrent at each wavelength to decrease nearly to zero. It can then be considered that trapped charges at each energy are completely released. The charge distribution can be obtained by the integrals of PSD currents at each wavelength [152]. As an example, the trap distribution for polyethylene film using the step scanning method is represented in Figure 15.

Improvement of the understanding of charge trapping phenomena can also be obtained by combining PSD and other techniques as space charge measurements, since PSD current is closely related to the trapped charge distribution in both energy and space [26,161]. One can also cite a technique called Exhaustive Photo-Depopulation Spectroscopy (EPDS) consisting in realizing Capacitance versus Voltage (C-V) measurement coupled to photo-discharge. This can be a way to overcome some of the problems found in photo-discharge measurements [162].

As mentioned by van Turnhout [49], optical stimulation can be used directly as a diagnostic tool for trap-level spectroscopy in those materials in which light efficiently releases electrons and holes from their traps. Phosphors used as scintillators have been particularly considered through the PSD technique [163] and extended to Optically Stimulated Luminescence - OSL, as briefly described in the next section. 


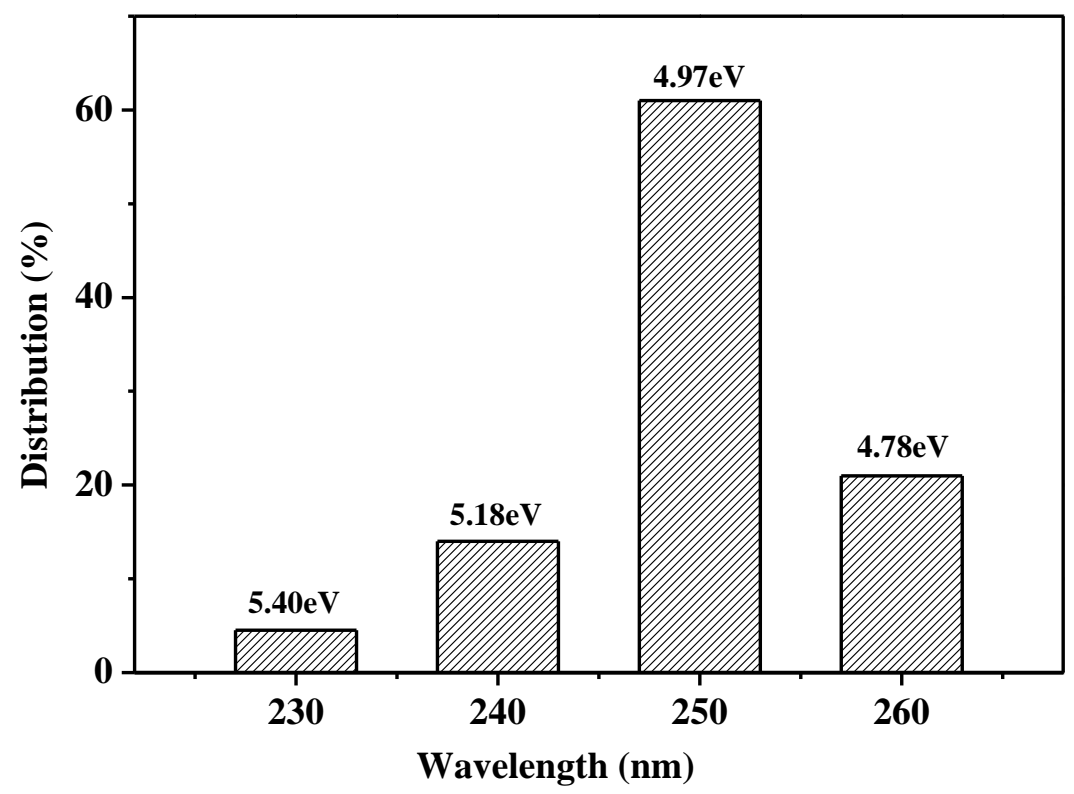

Figure 15. Trap distribution in polyethylene by the step scanning method.

Reproduced from [152]. (C) IOP Publishing Ltd. All rights reserved.

Over the years, the PSD method has been applied to various organic and inorganic materials. Table 3 summarizes some representative results from the literature on PSD method applied to inorganic and organic dielectric materials. In the field of polymers, Polyimide has notably been considered, for its sensitivity to photon stimulation, imparting it with photoinjection and photoconduction properties [159]. Trap depths are particularly large in those polymers containing aliphatic chains, such as PP, PE or PTFE. Conjugated polymers as Polyimide (though the results from different sources are not consistent) or Polycarbonate exhibit comparatively lower trap depth, and PET falls in-between. In general, trap depths are substantially lower in inorganic materials compared to polymers.

\begin{tabular}{|c|c|c|c|c|c|}
\hline & Materials & Thickness & Trap filling method & PSD peaks/ energy band & Ref. \\
\hline \multirow{3}{*}{ 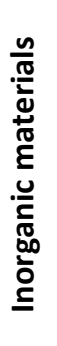 } & Anthracene crystal & $1.5 \mathrm{~mm}$ & $\begin{array}{l}\text { Photoinjection } \\
\text { (UV + electric field) }\end{array}$ & $\begin{array}{l}0.93 \mathrm{eV} \\
1.27 \mathrm{eV}\end{array}$ & $\begin{array}{l}\text { Brodribb et al } \\
\text { [149] }\end{array}$ \\
\hline & Tantalum oxide $\mathrm{Ta}_{2} \mathrm{O}_{5}$ & $480 \mathrm{~nm}$ & $\begin{array}{c}\text { Photoinjection } \\
\text { (UV + electric field) }\end{array}$ & $\begin{array}{c}0.4 \mathrm{eV} \\
1.1 \mathrm{eV} \text { (large and broad } \\
\text { band) }\end{array}$ & $\begin{array}{l}\text { Brodribb et al } \\
\text { [149] }\end{array}$ \\
\hline & Thermal $\mathrm{SiO}_{2}$ & $\begin{array}{c}2.5 \text { to } 4.2 \\
\mu \mathrm{m}\end{array}$ & Photoinjection & $2.13 \mathrm{eV}$ & $\begin{array}{c}\text { Thomas et al } \\
\text { [150] }\end{array}$ \\
\hline \multirow{4}{*}{ 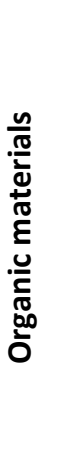 } & Polycarbonate & $3.5 \mu \mathrm{m}$ & $\begin{array}{l}\text { Photoinjection } \\
\text { (UV + High field) }\end{array}$ & $\begin{array}{l}\text { Broad band at around } 0.5 \\
\mathrm{eV} \text { and beyond } 1 \mathrm{eV}\end{array}$ & $\begin{array}{l}\text { Brodribb et al } \\
\text { [149] }\end{array}$ \\
\hline & $\begin{array}{c}\mathrm{Al}_{2} \mathrm{O}_{3} \text { nano powder - } \\
\text { polyethylene }\end{array}$ & $140 \mu \mathrm{m}$ & $\begin{array}{l}\text { Direct electric field } \\
\qquad(40 \mathrm{kV} / \mathrm{mm})\end{array}$ & $\begin{array}{c}4.97 \mathrm{eV} \text { for undoped LDPE } \\
\text { Trap levels are deeper with } \\
\mathrm{Al}_{2} \mathrm{O}_{3} \text { contents }\end{array}$ & $\begin{array}{l}\text { Zhu et al } \\
\text { [152] }\end{array}$ \\
\hline & Cellular polypropylene & $70 \mu \mathrm{m}$ & External field & $4.6,5.6$ and $6.3 \mathrm{eV}$ & $\begin{array}{l}\text { Mellinger et } \\
\text { al [26] }\end{array}$ \\
\hline & Polytetrafluoroethylene & $18 \mu \mathrm{m}$ & $\begin{array}{l}\text { Corona discharge or } \\
\text { electron beam }\end{array}$ & $4.6 \mathrm{eV}$ & $\begin{array}{l}\text { Mellinger et } \\
\text { al [26] }\end{array}$ \\
\hline
\end{tabular}


Review: Trap Spectroscopy in Polymer Dielectrics - Revised Version. Teyssedre et al 2021 J. Phys. D: Appl. Phys. https://doi.org/10.1088/1361-6463/abf44a

\begin{tabular}{|c|c|c|c|c|}
\hline $\begin{array}{l}\text { Polyethylene } \\
\text { terephthalate }\end{array}$ & $16 \mu \mathrm{m}$ & Corona discharge & $4.1 \mathrm{eV}$ & $\begin{array}{l}\text { Mellinger et } \\
\text { al [26] }\end{array}$ \\
\hline Polyimide & $25 \mu \mathrm{m}$ & $\begin{array}{l}\text { Direct electric field } \\
\qquad(40 \mathrm{kV} / \mathrm{mm})\end{array}$ & $\begin{array}{c}4.05 \mathrm{eV}(300 \mathrm{~nm}) 3.65 \mathrm{eV} \\
(340 \mathrm{~nm}) \text { and } 4.9 \mathrm{eV}(253 \\
\mathrm{nm}) \text { bands }\end{array}$ & $\begin{array}{l}\text { Yue et al } \\
\text { [61] }\end{array}$ \\
\hline Polyimide & $50 \mu \mathrm{m}$ & $\begin{array}{l}\text { Corona discharge or } \\
\text { electron beam }\end{array}$ & $\begin{array}{c}2.67 \mathrm{eV} \text { (broad peak) and } \\
3.20 \mathrm{eV} \text { (shoulder) }\end{array}$ & $\begin{array}{l}\text { Ma et al } \\
\quad[28]\end{array}$ \\
\hline Polyimide & $30 \mu \mathrm{m}$ & $\begin{array}{l}\text { Direct electric field } \\
(40-50 \mathrm{kV} / \mathrm{mm})\end{array}$ & $\begin{array}{l}\text { Mainly distributed in the } \\
\text { range of } 1.92 \mathrm{eV}-3.88 \mathrm{eV} \text {. }\end{array}$ & $\begin{array}{l}\text { He et al } \\
\text { [48] }\end{array}$ \\
\hline $\begin{array}{l}\text { Low Density } \\
\text { Polyethylene }\end{array}$ & $70 \mu \mathrm{m}$ & $\begin{array}{l}\text { Direct electric field } \\
\qquad(50 \mathrm{kV} / \mathrm{mm})\end{array}$ & 4.80 to $5.90 \mathrm{eV}$ & $\begin{array}{l}\text { He et al } \\
\text { [164] }\end{array}$ \\
\hline $\begin{array}{l}\text { Bi-oriented } \\
\text { Polypropylene }\end{array}$ & $18 \mu \mathrm{m}$ & $\begin{array}{l}\text { Direct electric field } \\
\qquad(165 \mathrm{kV} / \mathrm{mm})\end{array}$ & $\begin{array}{l}\text { Peak at } 3.1 \mathrm{eV} \text { (broad) and } \\
\text { double peak } 5.4 \mathrm{eV} / 6.1 \mathrm{eV} \\
\text { (narrow) }\end{array}$ & $\begin{array}{l}\text { Boudou et al } \\
\text { [165] }\end{array}$ \\
\hline
\end{tabular}

Table 3. Non exhaustive PSD results for organic and inorganic insulating materials reported by different researchers.

\subsection{Optically stimulated luminescence}

The basis of OSL measurement is to stimulate a pre-irradiated sample with light of a selected wavelength and to monitor the emission from the sample at a different wavelength. Different modes of stimulation, ranging from continuous wave, linear modulation and pulses are available. The method was reviewed by McKeever [166]. OSL is a relatively new technique that has been developed over the last 20 years for dosimetry purpose [167]. The idea behind OSL is to propose a faster and easier readout of information in dosimetry than TL can provide, with light excitation replacing the generation of a linear heating rate. As shown for previous methods for probing traps, a cascade of events can be at play, and models for analyzing the induced light are various. The light decay does not follow simple exponential decay, the curve shape being dependent upon sample, absorbed dose, wavelength of stimulation and temperature. OSL under CW illumination can be considered as a variant of chargerecombination Induced luminescence described in section 4.3. Notably, a decay kinetics of the same shape as shown above was reported $[168,169,170]$ :

$$
I(t)=A(1+B t)^{-p}
$$

where $p$ is in the range 1 to 2 and $A$ and $B$ are constants, possibly temperature-dependent.

OSL was reported to be sensitive not only to recombining defects but also to the band tail states to which the electrons are excited [170,171]. An advantage of OSL put forward compared to TL is to avoid generating a reproducible heating scheme, along with avoiding thermal quenching effects [166]. The method anyway reveals that the response is temperature dependent, and a number of processes explain this temperature dependency: the fact that the charges recombination can be via the conduction band or through donor-acceptor like process, the mediation of shallow trap levels and the competition with non-radiative de-activation processes [168]. Therefore, the OSL is either enhanced or reduced when the temperature is increased. These features show that the competition of all these processes should be resolved in order to access to an estimation of trap depth based on such method. 


\section{Discussion}

\subsection{Distributed traps in thermostimulated techniques}

In inorganic materials, activation energies obtained by TS techniques could be identified in terms of defects, being a single kind of defect or as discrete distributions [172]. Owing to the disorder introduced into polymeric materials, the activated processes necessarily involve some distribution in energy. This has led to the development of different variants of model for glow curves involving a distribution in energy. Whereas the direct problem, i.e. computation of a TS peak based on a given distribution is tractable, the inverse problem, i.e. the identification of the distribution in processes (time constants and amplitude) at the origin of a discharge peak is far from being simple. Based on two examples, we would like here to point on the difficulty in handling distributed processes in TS methods.

TSDC has been used to analyze relaxation processes in polymers: the processes, corresponding to local motions of polymer chain or to segmental motions associated with the glass transition, do not obey single Debye-like relaxation process and it is generally admitted that a distribution in relaxation times is at play [173]. On fundamental aspects, there is still some controversial about reasons for nonexponential rule of the relaxation associated to the glass transition temperature [174, 175]. Similar reasoning has been adopted for trap spectroscopy and a continuous distribution in trap depth could be produced from glow TSDC peak supposing the pre-exponential factor is fixed for all contributing processes [176].

One method put forward to analyze such complex response consists in using fractional polarization in TSDC, i.e. apply the polarization in a narrow window in time/temperature such as to isolate a narrow distribution in relaxation times $[56,177,178]$. A series of 'elementary' TSDC peaks is obtained, each of them being analyzed as a single Debye-like process. Then, activation parameters can eventually be used to obtain a continuous distribution in relaxation times. Such analysis was achieved with changing the width of the temperature window, from 0 , to 5 at $10^{\circ} \mathrm{C}$ [125]. An appealing result was obtained: the activation energy range depends on the width of the polarization window, i.e. on the underlying distribution of relaxation times. The narrower the distribution in relaxation times, the larger the estimated activation energy. This trend tends to show that the energy obtained in case of distributed processes is clearly dependent on the distribution itself. In the same vein, Chen [179] demonstrated, using numerical simulations, that very low effective values of the activation energy and frequency factor may be evaluated due to the occurrence of two or more TL peaks in close vicinity to each other.

Further argument about this question is brought by recent computations of glow TL curves achieved with the Gaussian distribution in activation energies and considering different widths in the distribution [180]. Once TL glow curves were obtained, it was analyzed by the initial rise method. Figure 16 illustrates the distributions taken and the activation energy obtained (in red) for each case. Surprisingly, the deduced activation energy falls into the shallowest values in the distribution whereas we could expect that the mean activation energy is obtained. Only with very narrow width in activation energy distribution ( $1 \%$ of the mean value) a good estimate of the mean value could be obtained. It must therefore be concluded that in case of distributed processes, most certainly an underestimate of the real activation energy is produced. 

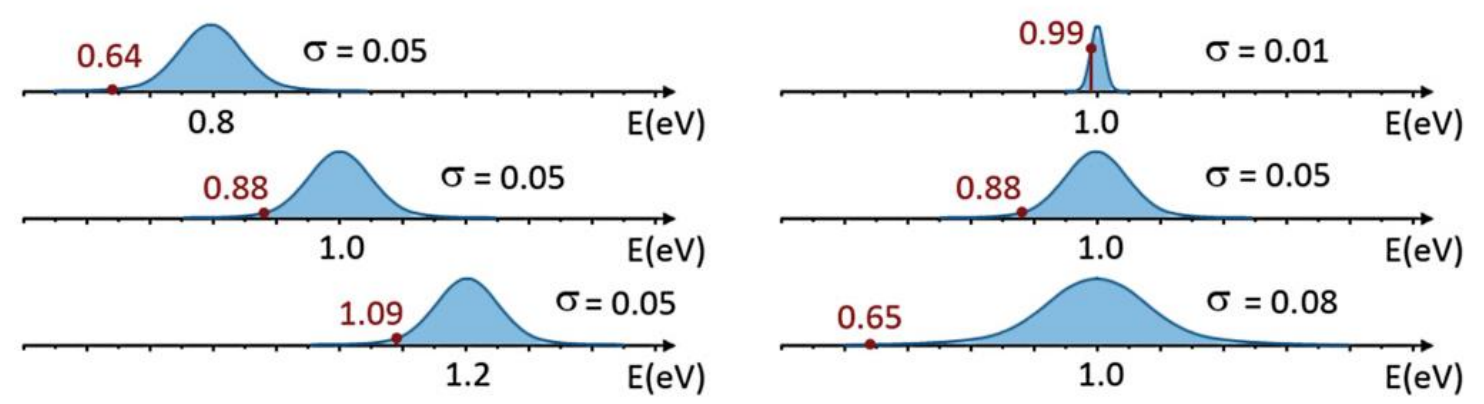

Figure 16. Activation energies (in red) predicted with using the initial rise method in computed TL curves. The initial distribution was Gaussian with different width $\sigma$. In all cases, the initial rise method provides the shallowest level in the distribution. Reprinted figure with permission from [180], Copyright (2013) by the American Physical Society.

\subsection{Trap depth vs. molecular motions}

In our point of view, molecular motions in polymers may make the analysis of trap distribution more complicated than in other solid insulations.

We have shown that the activation energy of the process, the trap depth, can in principle be deduced from thermostimulated curves. However, the peaks observed in TL or TSDC curve can also correspond to the physical disappearance of the traps, at relaxation temperatures that correspond to the onset of local molecular motions of the polymer. In that case, the deduced activation energy does not necessarily represent trap depth.

Dobruchowska et al [181] investigated in detail this phenomenon, known as 'wet dog' effect. The comparison was made between the positions of the TL maxima and the secondary molecular relaxations in different poly(alkyl methacrylate)s and polystyrene doped with various chromophores: pyrene, carbazole and perylene. Though the strength of the TL peak for a given relaxation depends on the nature of the dopant, it was confirmed that most of the TL maxima correspond well to the onset of molecular motions, what affords evidence for a significant contribution of the molecular motions to the charge carrier detrapping process. However, the activation energies obtained in TL tend to be substantially lower than that deduced from impedance spectroscopy. It was argued that the activation energy determined from the TL experiment is in fact the energy required to initiate releasing of the charge carriers from the traps, but not necessarily the energy that is sufficient to activate the entire molecular relaxation process. Another example of close correlation between TL peaks and relaxation processes was reported for Polyethylene terephthalate [182].

As quoted by Perlman [52], the detrapping of charge may be a dual process that arises from both a lowering of trap depth with molecular motion plus thermal excitation of the charge out of the trap. Since charge release in polymers is so intimately connected with molecular motion, it may be that some of the activation energies mentioned are not those due to charge affinity of various atoms, or groups of atoms, but rather are to be associated with molecular vibration and rotation.

A further complication is, in our opinion, the problem of the variation of the quantum efficiency of the emission as a function of temperature in case of TL analysis. Luminescence techniques have as drawback to be very sensitive to the nature and environment of emitting groups, therefore rendering quantitative analysis difficult, especially for materials in the solid state. As far as polymers are concerned, the luminescence yield can be substantially temperature dependent [183, 184]. 
Phosphorescence, which corresponds to the relaxation of long-lived excited states involving spin forbidden transition, is sensitive to energy relaxation by non-radiative pathways like quenching by molecular oxygen. The emission intensity decreases with the increase in temperature as mobility of chains makes the interactions with dissolved molecular oxygen easier [185]. In this way, luminescence processes can be sensitive to molecular motions.

Up to now, though some works report on the quenching effects for inorganics [140] it seems not to have been considered in the literature on TL in polymers. It is probably due to the fact that wavelengthresolved spectra have been reported scarcely $[186,187]$, such that little was known about the origin of the emission. As we have shown that the luminescence involved in $T L$ is phosphorescence, and that the phosphorescence itself varies strongly with temperature because of quenching effects, there is no reason to consider that the quantum efficiency of $T L$ is not dependent on the temperature. Thus, the lower the temperature is, the greater the probability for a recombination event to be radiative.

Figure 17 shows the TL curves obtained for crosslinked polyethylene before and after correction from the temperature dependence of the main emission at about $450 \mathrm{~nm}$ in photoluminescence experiments [188]. The difference in the two thermograms is considerable. The main peak occurring at low temperature becomes a shoulder after correction. For the thermally treated sample, two transition regions appear at about $-120^{\circ} \mathrm{C}$ and $-60^{\circ} \mathrm{C}$. It is tempting to associate these with the $\gamma$ and $\beta$ relaxations of polyethylene that occur in the respective temperature regions according to dynamical mechanical analysis [189].

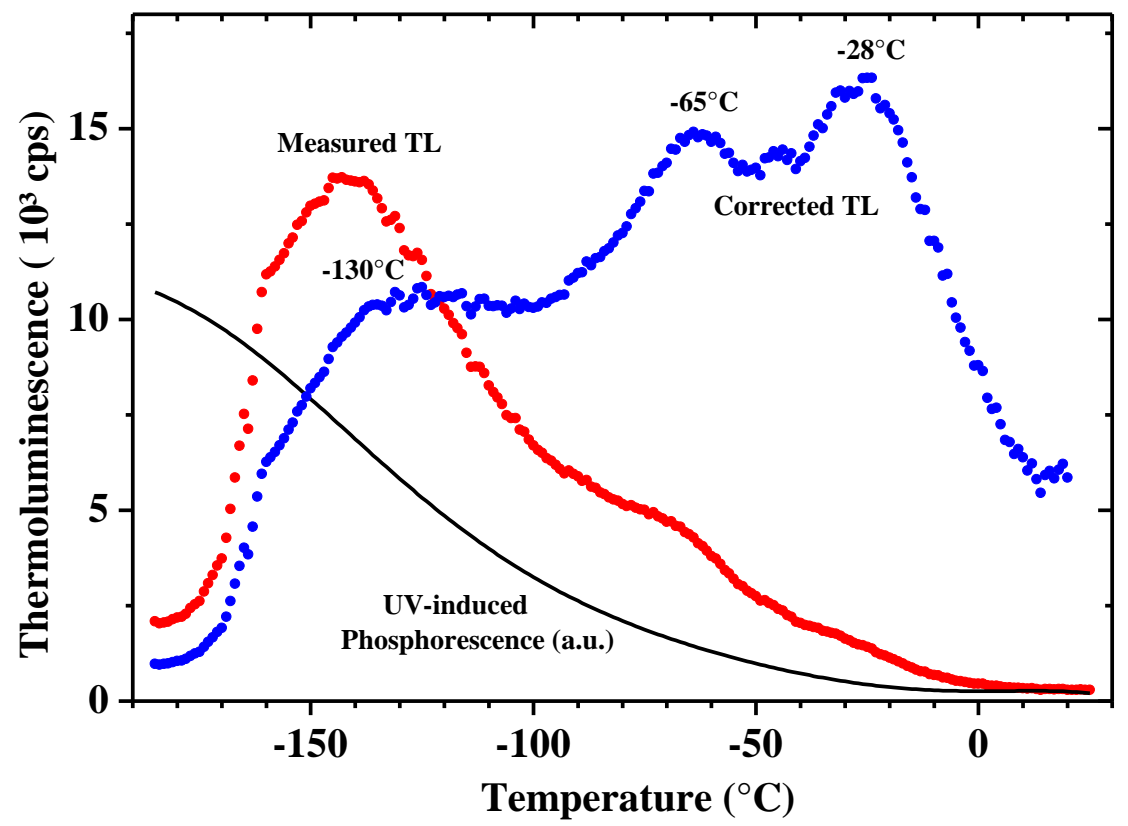

Figure 17. Thermoluminescence curve obtained after excitation of crosslinked polyethylene by a plasma discharge. Reprinted by permission from Springer Nature Customer Service Centre GmbH: [Springer] [188] (2002).

This goes with questioning about the intimate relation between the activation energy deduced from detrapping and for dipolar chain motion reported by Kojima et al for PET [22]. From a practical point of view, this has no real consequence when the information expected is the effective depth controlling the stability of charges and the kinetics of detrapping. It should be verified, through e.g. a checking of consistency of the response obtained by other techniques, like isothermal methods, that processes are 
effectively thermally activated. Of course, on a physical standpoint the significance is not the same whether molecular motions surrounding trapping center or electronic levels associated with a given molecular group control charge stability. This difference can perhaps be the source for the apparently conflicting results obtained from thermal and optical excitations in terms of trap depths.

\subsection{Difference between thermal and optical excitation techniques}

Recently He et al [164] reported on a substantial difference in trap energies, i.e. $0.8 \mathrm{eV}$ vs. $4.0 \mathrm{eV}$ for thermal vs. optical excitation, even though the discharge process apparently arises from the same distribution of traps. The effect was explained by a "thermal erosion" of traps in the case of thermal (TSC) method, and it was considered that PSD could more truly characterize the intrinsic properties of traps of the polymer. The term "erosion" is a big vague and it can be supposed it corresponds to the thermal destruction of traps. Instead of using photobleaching for neutralizing occupied shallower traps, Suzuoki et al [190] used partial heating technique followed by PSD measurements in the correlation between experiments. Again, the energy obtained by optical excitation appeared larger than under thermal excitation. They interpreted the differences in activation energy obtained by initial rise method in TSC and PSD as due to direct traps modification induced by molecular motions under the effect of temperature rise while optical methods probe a frozen configuration.

Figure 18 shows several possible optical mechanisms occurring in insulators [166]. Transition of type (4) is that corresponding to the optically stimulated release of previously trapped charges. This is of relatively low energy compared to the band gap.

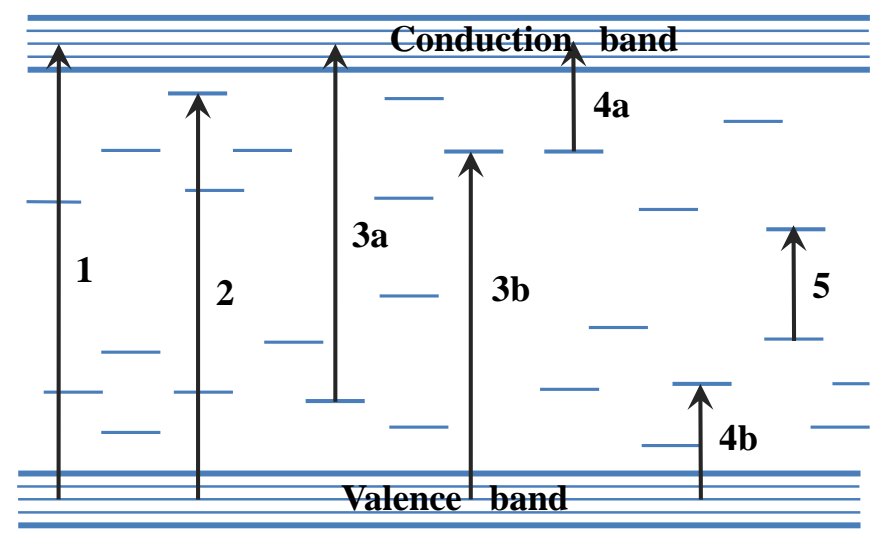

Figure 18. Possible optical absorption transitions in an insulator: (1) ionization (excitation across the band gap); (2) exciton formation; (3) defect ionization; (4) trap ionization; (5) internal intra-center transition.

Reprinted from [166], Copyright (2001), with permission from Elsevier.

In inorganic crystals, it is considered that the optical trap depth $E_{0}$, i.e. the defect ionization energy can be substantially larger than the thermal trap depth $E_{t}$ by an amount corresponding to the lattice phonon vibration energy. An approximation is given as $E_{0} / E_{t}=\varepsilon_{0} / \varepsilon_{s}$, where $\varepsilon_{0}$ and $\varepsilon_{s}$ are the high frequency and static dielectric constants. Braunlich et al [123] depicted a scheme for optical and thermal ionization energies of an occupied trap based on Franck-Compton principle in case of strong electron-lattice coupling. It applies to the case of large changes in equilibrium lattice coordinates and energy of the defect state during capture or ejection of the electronic charge carrier. The thermal 
ionization energy is lower than the optical one by an amount related to the lattice reorganization energy. A fundamental aspect to treat in optical excitation is the efficiency of the excitation of charged traps. This is traditionally defined as capture cross-section $\sigma_{0}(\lambda)$ when dealing with semiconductors. Grimmeiss et al $[191,192]$ discussed optical versus thermal activation energy- or phosphors. The absorption coefficient is directly related to $\sigma_{0}(\lambda)$. For deep levels in and insulator and parabolic band, an expression of the following form has been used [166]:

$$
\sigma_{0}(\lambda)=C \sqrt{E_{0}} \frac{\left(h v-E_{0}\right)^{3 / 2}}{h v\left(h v-\eta E_{0}\right)^{2}}
$$

Here $h v$ is the excitation energy and $\eta=1-m_{0} / m *$ with $m_{0}$ the free carrier rest mass and $m^{*}$ the effective electron mass. Figure 19 shows the computed cross-sections considering an optical gap $E_{0}$ and different values of $\eta$. It shows that the peak in absorption wavelength can be at significantly larger energy than $E_{0}$. This is a possible contribution to the relative large values (overestimates) of the measured trap depth with optical excitation. However, in general, such photoionization cross-sections are not documented in the literature.

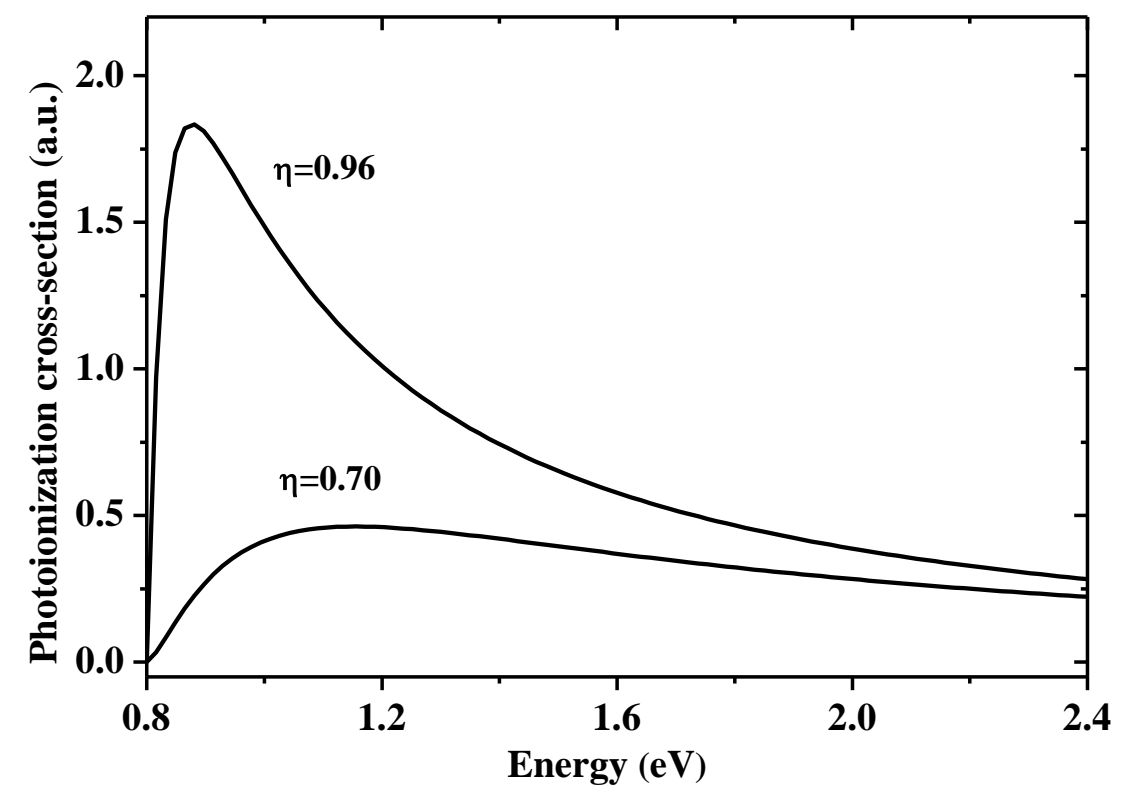

Figure 19. Optical photoionization cross-section spectra computed using Eq. (16)

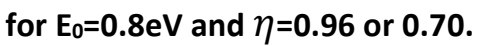

Another feature to account for is the specificity of energetic levels into organic materials. Because of the relatively small interactions between chains, molecules essentially retain their identity in the condensed state and thus the solid-state description and the related band structure do not necessarily apply with the same strength as for inorganics. This coupled with the fact that there is fluctuation in the local structure and composition may make transport and optical properties substantially dependent on disorder and localized states more than extended states [193]. Example of specificities of polymers is with side groups containing macromolecules as polystyrene: though they contain $\pi$ electrons, there is no conjugation path along the main chain [194]. These groups should contribute to the optical and electronic properties of the material by introducing internal transition level, depicted as (5) in Figure 18. The photoemission threshold is at about 7eV, but electron energy losses appear in the 4-7 eV range presumably due to electronic excitation as revealed by low energy backscattered 
electron spectroscopy [195]. Because of the narrow bands for extended states characteristic of inorganic materials, on the one hand, and of the disorder introduced in the material, the cross-section spectrum can be something different from the one plotted in Figure 19. Certainly the investigation of the energetics of such charge states would be an interesting aspect to be treated by chemical physics methods. Secu and Polosan [196] used XPS measurements combined to DFT computations to explain the double peaks in TL from Alq3, an organic semiconductor: states acting as traps would be relaxed triplet states from two Alq3 isomers. Upon thermal activation, electrons would be excited to the conduction band and would relax subsequently to produce photoluminescence.

The differences and consistencies between unstimulated and stimulated techniques are worth addressing. In luminescence, we have shown that isothermal and thermally stimulated responses provide the same emission spectra, pointing to the same nature of recombining centres. For the case of dipolar processes in polyethylene, Fischer and Rohl $[197,198]$ proposed a model with a distribution in activation energies which very satisfactorily accounted for both isothermal discharge currents and TSDC. Also Hoang et al [199] recently reported on consistent results in trap depth between isothermal discharge currents and TSDC from the literature. Though in fact unstimulated methods involve thermal release of traps like do thermally stimulated techniques, comparison in terms of trap distribution is not so common. Charging for TSDC for example is generally achieved at high temperature and the stored charge is frozen by cooling. In unstimulated methods, charging and discharging are done in isothermal conditions. The charge distributions, which determine the hypotheses to be used for analyzing the charge decay can be different. Though unstimulated methods are easier to implement, there is no external stress applied on the charges, more hypotheses need to be done about discharge mechanisms and the response is tributary to the kinetics of processes at the test temperature. Comparatively, stimulated methods provide a more global and fast response and may help in identifying overlapping contributions.

\subsection{Information gained on the nature of traps}

Most of the techniques described above give access to the energy distribution of trapping levels without pointing to the chemical nature of the trap. Only those techniques relying on luminescence detection could provide a way to infer their nature through the acquisition and analysis of the emission spectrum. It could be TL or RIL which in fact yield the same emission spectra [188] demonstrating the same deep traps act as recombination center in the two experiments.

\subsubsection{Polyolefins}

The charge recombination-induced luminescence spectrum shown in Figure 8 for commercially available polypropylene is remarkably similar to the one obtained for polyethylene [21]. This feature is strong indication that the same species act as recombination centers in both materials. Identification of the electronic transitions involved in recombination has been done considering the photophysical/photochemical properties of these materials available from an abundant literature $[200,201,202,203]$. The fluorescence emission, at about $340 \mathrm{~nm}$ in photoluminescence was associated to the presence of unsaturated carbonyls of the enone type (- $\mathrm{CH}=\mathrm{CH}-\mathrm{CO}-)$. Phosphorescence emission ( $450 \mathrm{~nm}$ ) was ascribed to the presence of unsaturated carbonyls of the dienone type $(-\mathrm{CH}=\mathrm{CH}-\mathrm{CO}-\mathrm{CH}=\mathrm{CH}-\mathrm{R}$ or $-\mathrm{CH}=\mathrm{CH}-\mathrm{CH}=\mathrm{CH}-\mathrm{CO}-\mathrm{R})$ that also provide recombination centers. 
The structured phosphorescence emission and the excitation conditions are related to the coexistence of $\left(\pi, \pi^{*}\right)$ and $\left(n, \pi^{*}\right)$ electronic transitions in poly-enone sequences since carbonyls have a non-bonding orbital $n$ due to a nonbonding $\pi$ electron on the oxygen atom [204], 205, 206].

Charge recombination on these chemicals gives rise to light emission mediated by specific electronic transitions as compared to photo-induced phosphorescence, peaking at $510 \mathrm{~nm}$ and extending over the long wavelength tail of the phosphorescence spectrum. The later does not involve ionization of the center. Poly-ene sequences $-(\mathrm{CH}=\mathrm{CH})_{n}$ - provide hole traps, with energy levels located in between $0.5 \mathrm{eV}(\mathrm{n}=1)$ and $1 \mathrm{eV}(\mathrm{n}=2)$ from the top of the valence band [207]. Poly-enone sequences $-(\mathrm{CH}=\mathrm{CH}) \mathrm{n}^{-}$ CO- are polar and provide deeper traps located at about $1 \mathrm{eV}(n=1)$ from the band edge for both electrons and holes [207]. These polar sequences provide adequate sites for recombination processes. The reason why charge recombination on such luminescent centers would give rise only to phosphorescence emission in the region of the lowest lying triplet states lies in the configuration of the orbitals in carbonyl compounds [205, 206].

\subsubsection{Crosslinked and grafted polyethylene}

The method of identification of deep trapping centers using RIL was pushed by experiments on chemically modified polyethylene. Specific materials were prepared containing chemicals, either grafted or not on the polymer backbone. The by-products formed by peroxide crosslinking of PE, see Figure 20a, constitute one of the families $[188,208]$.

We also investigated reaction products of a commercial antioxidant [20] and finally specific molecules grafted onto polymeric chains [104] with expected role of voltage stabilizer $[209,210]$ that could be interesting for DC applications. We give the example of two chemically modified XLPE, one with grafted styrene at $5 \%$ and the other with a grafted benzophenone derivative at $1 \%$. The chemical formulation of the grafted species is given in Figure $20 \mathrm{~b}$.

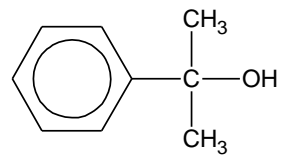

cumyl alcohol

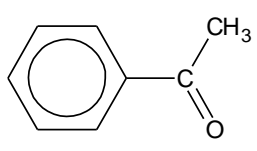

acetophenone

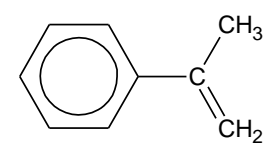

$\alpha$-methylstyrene (a)

(b)

grafted benzophenone derivative

grafted styrene

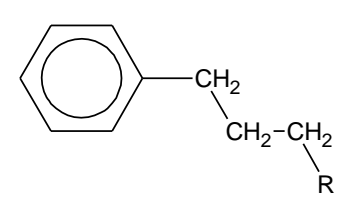

Figure 20. Chemical formulation of (a) main by-products of cross-linking reaction with dicumylperoxide as crosslinking agent and (b) of the grafted species investigated as voltage stabilizers. 
The RIL spectra are given in Figure 21 with a comparison to the photoluminescence spectra. It appears that, for grafted groups, the RIL and photoluminescence spectra (dominated by phosphorescence in all cases) match very well. It is therefore clear that these chemicals play the role of deep trapping levels. In case of 'pure' XLPE material (and of benzophenone-grafted LDPE) the phosphorescence spectrum contains structures spaced in energy steps that correspond to the aromatic $\mathrm{C}=\mathrm{O}$ vibrational frequency at about $1730 \mathrm{~cm}^{-1}$. This structured spectrum appears typical of acetophenone [208]. The vibronic structure is not so evident in the RIL spectrum. A peculiar feature of acetophenone is that $\mathrm{n}$ $\pi *$ and $\pi-\pi *$ triplet levels are close lying, implying significant change in phosphorescence lifetime, as well as quantum yield, depending on the environment $[211,212]$. The emission from $\pi-\pi *$ states of substituted acetophenone has a more diffuse structure, and a redshift of the order of 10-20 nm may appear $[213,214]$. A possible explanation of the current photoluminescence results is that when acetophenone is present as a free species, emission would be mostly from an $n-\pi *$ state. When acetophenone is bound, relaxation would involve $\pi-\pi *$ states. It is suggested that the RIL spectrum would reflect the formation of $\pi-\pi *$ states, again as the lowest lying triplet states available and mainly bounded acetophenone would be active as trapping center.

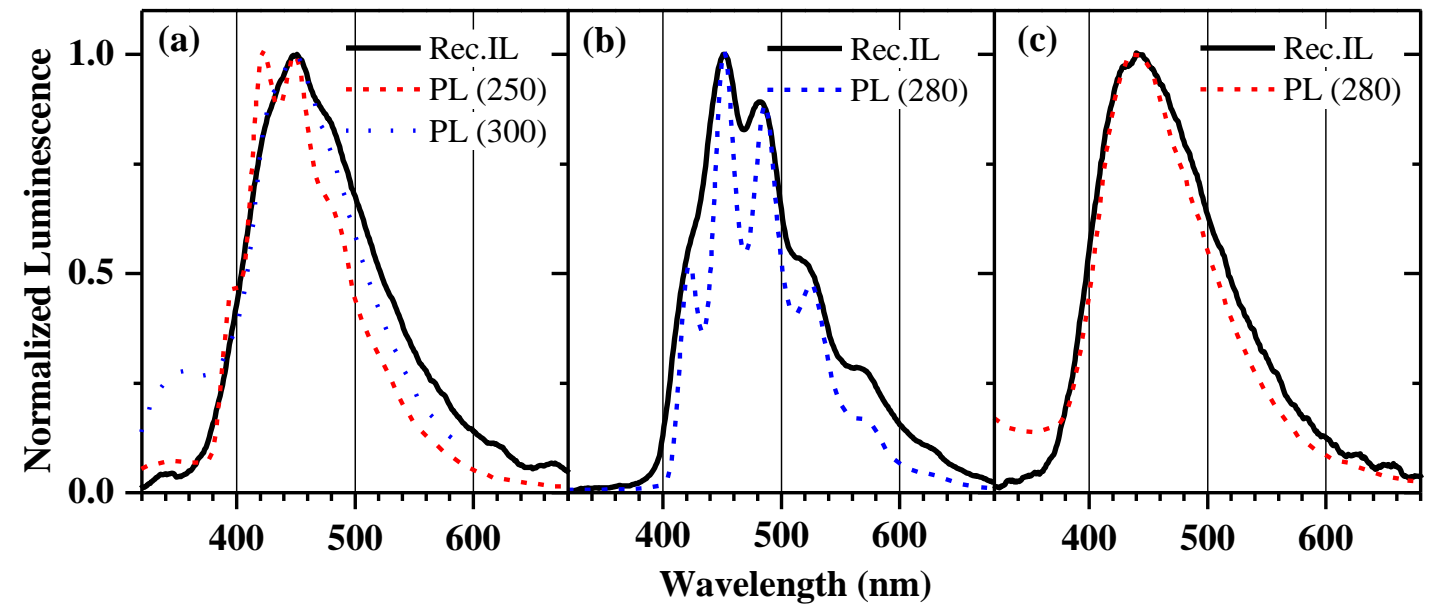

Figure 21. Comparison of the recombination-induced luminescence (Rec IL) and photoluminescence spectra (PL with indication of excitation wavelength) for (a): XLPE, (b): XLPE with grafted benzophenone, (c): XLPE with grafted styrene groups.

The study on different reaction products of Santonox, a common antioxidant for polyethylene, revealed that part of the luminescence spectrum of XLPE, and even essentially the all spectrum in case of thermally treated films, can be explained by the introduction of the antioxidant and subsequent reaction of it [20]. Those molecules are also very active in RIL. Therefore, peroxide by-products are not the only chemicals to be considered when attempting to identify trapping centers in XLPE. The fact that crosslinking agents are easier to investigate as they can move relatively freely within the insulation and their chemistry is relatively simple may explain why they have been more specifically considered. On the other side, AO-related reaction products constitute immobile, non-volatile chemicals, homogeneously distributed within the insulation. They can assist conduction and provide also deep trapping sites for charges, as inferred also considering space charge and conduction measurements [215].

It is noticeable than in the investigated family of polyethylene and extending to polypropylene, RIL seems to involve only triplet states. In photoluminescence, the probability of creating a triplet state 
depends on the rate of intersystem crossing from singlet states. It can be very low, especially when the difference in energy between the singlet and triplet states is large. Conversely, the probability of creating a triplet excited state in a charge recombination event is theoretically three times as large as the probability of creating a singlet excited state [216]. This means that species known to be nonphosphorescing may be so because of excitation limitations under UV irradiation and they may behave in a completely different way regarding emission yield excited by electrical charges.

\subsubsection{Polyethylene naphthalate}

In a polymer like Polyethylene naphthalate containing in-chain chromophores, the emission spectra are a priori more simple. In photoluminescence of PEN at room temperature, cf. Figure 22a, mainly fluorescence is observed peaking at $430 \mathrm{~nm}[112,217,218]$. When cooling to low temperature (around $-120^{\circ} \mathrm{C}$ ), phosphorescence, i.e. relaxation of triplet states is clearly revealed in the form of a doublet $(580,618 \mathrm{~nm})$. The fluorescence appears blue shifted and several bands appear in it, most probably due to the vibronic structure of the emitting groups. Electroluminescence under DC field, Figure $22 \mathrm{~b}$ shows very clear temperature dependence: at low temperature it is dominated by the phosphorescence of the material (relaxation of triplet states) while at room temperature new bands appear, and were ascribed to the formation of degradation by-products under the effect of the field. These effects have been discussed elsewhere [219]. The case of RIL spectra is interesting to comment here. The total light decay following plasma interaction at low temperature follows remarkably the same kinetics as when switching off the field, cf. Figure 9 [116]: The decay can be fitted to a hyperbolic law ( $3^{\text {rd }}$ term in Eq. (6)) without need to add other contributions [220]. It was not clear if the spectrum obtained in different time intervals during the isothermal decay could evolve in time.

The main difference compared to the EL spectra obtained either under continuous excitation or during the decay is the presence of a clear fluorescence emission. The absence of fluorescence in the EL spectrum has been related to the possible excitonic dissociation under the effect of the field [218, 221]. However, this is not really supported by an invariance of the spectrum during the decay following excitation under field, except if very large field is retained locally long after DC application because of space charge-induced field. Another possibility is EL dominated by processes occurring near the surface of the material, where a degraded layer may be present. However, this latter possibility is supported neither by the difference between EL spectra under AC/DC stress nor by the results for RIL spectra: under plasma excitation, the very near surface is probed and if such degraded layer was present it should be revealed. One may argue on the fact that in these experiments and in the particular case of PEN the UV from the discharge produce ionization of the material deeper in the bulk and that part of the excitation is made this way.

The results from TL are further appealing. In these experiments, the heating was not tightly controlled and only trends about qualitative evolution of the signal during heating could be obtained. We can see in Figure 22c that during heating, the ratio of phosphorescence to fluorescence contributions is increasing. This behaviour appears counter-intuitive if one considers the evolution of the response in EL or PL versus temperature. 


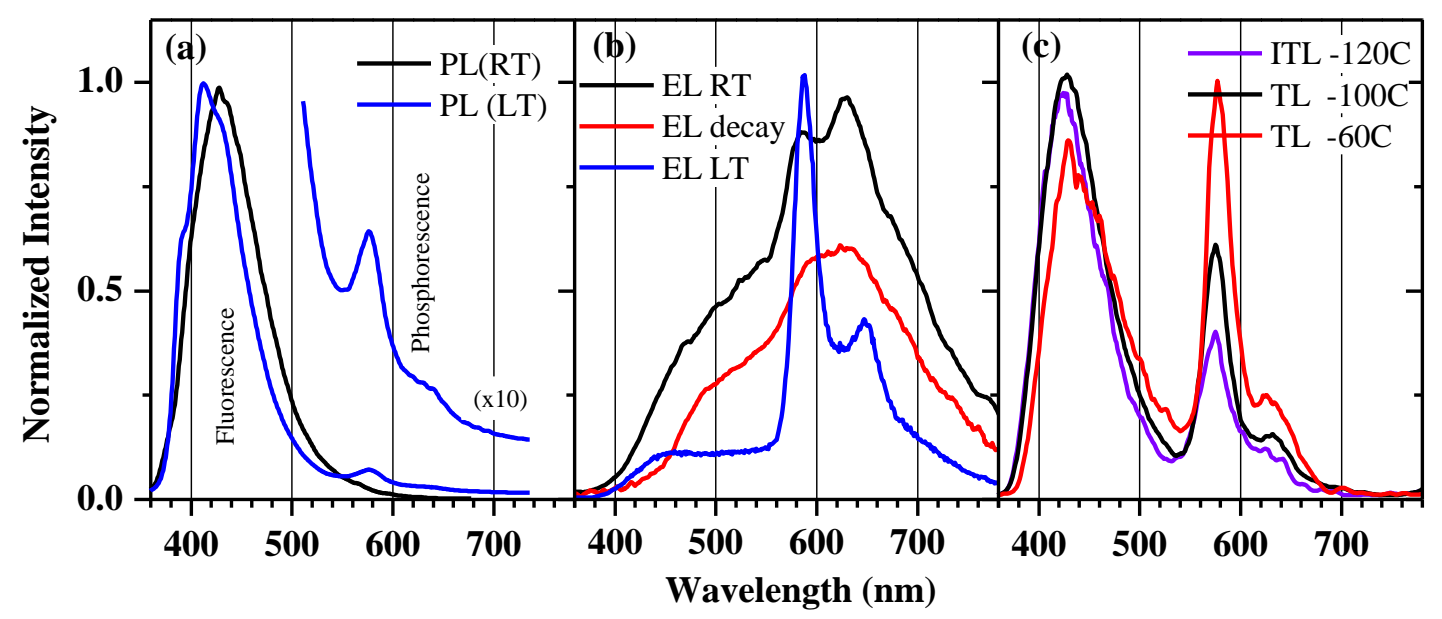

Figure 22. Luminescence spectra of PEN using various excitation sources. RT stands for room temperature, $\mathrm{LT}$ for low temperature (about $-120^{\circ} \mathrm{C}$ ). (a) Photoluminescence with excitation at $260 \mathrm{~nm}$; (b) Electroluminescence spectra under DC field of $310 \mathrm{kV} / \mathrm{mm}$. In red: spectrum recorded during the decay; c) PIL spectra of PEN: ITL 3 min after end of plasma interaction and during heating $(\mathrm{TL})$ at different temperatures (approximate temperatures with $20^{\circ} \mathrm{C}$ intervals).

An explanation for these qualitative differences in the charge-induced response of the material comes from the density of excited states: excited states can be produced by impact excitation if energetic carriers are available or by charge recombination -with, following simple spin multiplicity rules [222] a probability of $75 \%$ for producing a triplet state rather than singlet state. A further route for fluorescence is the possibility of delayed fluorescence following excitation of triplet states. Triplet excitons can take part in a bimolecular annihilation process known as exciton fusion. This process can result in the formation of one singlet exciton from two triplet excitons [223, 224]. As the charges are released, the density of triplet excited states would be decreasing, hence the delayed fluorescence decreasing faster than the phosphorescence.

\section{Summary and Outlook}

With the multiplication of techniques and variants for probing trap distributions in dielectrics, the question of the validity of the hypotheses taken in the analysis and the consistency of the results obtained with different techniques clearly arose. When the detected signal is an electrical current, there is an obvious need to take into account the fate of charges from their traps to the detected electrode to obtain quantitative information. Most often as when dealing with TSDC technique or potential decay, the initial position of the charge and their nature are not known and retrapping processes are ignored. When using space charge measurement methods, the position of charges is accessible, but only in scarce works their effective motion is taken into account. In addition, part of the signal can be due to dipole motion and is not representative of trapped space charges. Luminescence detection does not require the motion of charges over significant distances to provide a signal related to charge detrapping. As the luminescence process involves a recombination centre, an extra information can be obtained through the emission spectrum, characteristic of the recombining centre, and presumably of the site stabilizing the charge. A drawback of luminescence is the difficulty in being quantitative as the emitted light is dependent upon the probability of having a 
radiative charge recombination. Combining TSDC and TL is a way to discriminate events related to charge detrapping.

The intrinsically disordered nature of polymers from both chemical and structural standpoints let obviously expect having traps of different characteristics coexisting. The fact that double top-hat distribution (though top-hat shape presumably reflects only a rough approximation of the actual distribution) can apparently fit potential decay as well as space charge decay $[39,79,101]$ in different conditions for the same material reflects this distributed nature. It also provides support to charge transport models based on two trap levels - deep and shallow - accounting for long lasting charge storage and for transport properties [12]. In the specific case of thermally stimulated methods, we showed that having overlapping distributed processes, as often appears in polymers, does lead to an underestimate of trap depths. Therefore, the energies obtained in this way should be considered as an apparent trap depth.

Irrespective of the method considered, a further issue to be solved is the quantitative aspect of the data obtained in terms of trap density. As stated in the introduction, what is reasonably accessible is an amount of occupied traps after a charging event. But the total trap density is difficult to approach. With the OD model applied to potential decay, trap densities spanning over 9 orders of magnitude are reported depending on materials [39, 90], which is not explained.

A very appealing question is the difference in trap depth that is generally obtained considering optical and thermal excitation. Several works achieving comparison on the ways of stimulating charge detrapping concluded on very substantial differences in the two approaches, and so far, there has been no convincing study reconciling the two approaches. On the thermal excitation side, it is argued that a 'wet dog' effect, i.e. detrapping due to dynamic destruction of the trap with the movement of the polymeric chains is at play, impeding the charge release through classical scheme with jump over the barrier representing the barrier depth. This view is supported by the consistency between relaxation temperatures of the polymer and temperature inducing charges release. On the optical excitation side, the difficulty is to estimate the actual efficiency of detrapping. This is traditionally defined as capture cross-section when dealing with semiconductors. The excitation cross-section is not necessarily at maximum for an energy corresponding to the trap depth. Because of the narrow bands for extended states characteristic of inorganic materials, on the one hand, and of the disorder introduced in the material, the excitation cross-section spectrum is not necessarily at maximum for the energy of the trap and can be shifted to higher energy.

Trap spectroscopy methods are really necessary today, with the area of nanocomposite and 'nanodielectrics' in which many questions are still open. Introducing nanocharges improves many of the properties of dielectrics, including an apparent reduction in space charge build-up and in electrical conductivity but so far there is no definitive explanation on the mechanisms. Space charge measurement methods can't reach the resolution expected to probe internal interfaces. Sophisticated methods as (true) trap spectroscopy can be one solution. Deeper charge traps when introducing nanocharges into polyethylene was reported by Hoang et al [199] as example and attributed to polymer nanofillers interfaces. More complex view on the impact of nanofiller amount on shallow and deep traps was recently given by Mi et al [225] based on TSDC data with possible interference with polymer morphology. Technically, trap spectroscopy methods can be applied to nanocomposites exactly in the same way as for polymer matrix since nanoscale resolution is not reached. For reaching 
convincing interpretation, it is important that the chosen method provides quantitative information on traps amount and it is still a difficult question. Besides, a combination of methods may help in distinguishing processes appearing near the electrodes, like field screening induced by deep charge traps, from those related to the dielectric bulk.

We see two lines of thinking for improving our understanding of the information gained by trap spectroscopy techniques.

First, coupling trap spectroscopy methods and space charge profile measurements may provide a stepby-step information on if -and how- charge redistribute in the course of scanning. For example, TSDC from dipolar processes can be distinguished from those from space charges release in this way [226]. Using space charge profile measurement methods having the required resolution, it may also be possible identifying the different candidate processes (photogeneration, detrapping, photoconduction) to photo-stimulated discharge, along with evaluating if the photoexcitation goes with material discharges, which is not necessary the case if one have in mind retrapping events occurring in OSL [168]. The approach could then be used to compare thermal and optical stimulation techniques using the same pre-charging conditions to evaluate if the same trapping centres are activated characterized by different activation energies or if the probed trap populations are not the same. The way electrodes used impact the response is worth investigating. Probably, the PSD method is the one the most sensitive to the electrodes configuration owing to the possibility of photoinjection for example, and the perturbation can be introduced both at the charging step and the probing step. For SPD, there is virtually no effect since experiments can be made on a free surface. Crossing the experiments can be a way to identify perturbations introduced by the type of electrodes used. In turn, changing the electrodes (and the polarity) can be a way to probe different kinds of traps.

For a different evaluation of trap depths, on must consider modelling methods based on molecular dynamics: such methods now have come to mature age, enabling to identify the ionization energy of a molecular defect in a particular chain arrangement. The methods formerly have been applied to probe various electron traps in polyethylene related to in-chain defects, and a discrete set of levels was computed, limited by the kind of defects considered, as discussed in section 2 . Out of chemical defect-containing polymers, the technique was applied to probe electronic properties of various polymers as Polystyrene, Poly(methyl methacrylate), or Poly(ethylene terephthalate) [227], fluorinated polymers [228] and Polyimide [229] that contain unsaturated bonds. It was also extended to interfaces in nanocomposites [230, 231]. A step forward with modelling approaches is being achieved with combining density functional theory and GW-BSE (GW-Belthe-Salpeter equation) in order to compute the optical properties of materials, in particular energy levels involved in excited states $[232,233]$. These approaches are very relevant to the problem of traps identification treated in this review, notably regarding photostimulated detrapping and luminescence processes upon charge recombination. 
Review: Trap Spectroscopy in Polymer Dielectrics - Revised Version. Teyssedre et al 2021 J. Phys. D: Appl. Phys. https://doi.org/10.1088/1361-6463/abf44a

\section{ORCID iDs}

$\begin{array}{ll}\text { Gilbert Teyssedre } & \text { https://orcid.org/0000-0001-6684-0262 } \\ \text { Feihu Zheng } & \underline{\text { https://orcid.org/0000-0002-1728-050X }} \\ \text { Laurent Boudou } & \text { https://orcid.org/0000-0001-5339-2042 } \\ \text { Christian Laurent } & \text { https://orcid.org/0000-0002-6625-2433 }\end{array}$

\section{References}

[1] Dissado L A and Fothergill J C 1992 Electrical Degradation and Breakdown in Polymers (London: IET)

[2] Teyssedre $G$ and Laurent $C 2005$ Charge transport modeling in insulating polymers: From molecular to macroscopic scale IEEE Trans. Dielectr. Electr. Insul. 12 857-875

[3] Mott N F 1977 Electrons in glass Contemp. Phys. 18 225-245

[4] Tessler N, Preezant $Y$, Rappaport $N$ and Roichman $Y 2009$ Charge transport in disordered organic materials and its relevance to thin-film devices: A tutorial review Adv. Mater. 21 2741-2761

[5] Stallinga P 2011 Electronic transport in organic materials: Comparison of band theory with percolation/ (variable range) hopping theory Adv. Mater. 23 3356-3362

[6] Mark P and Helfrich W 1962 Space-charge-limited currents in organic crystals J. Appl. Phys. 33 205-215

[7] Kryszewski W and Szymański A 1981 Space charge limited currents in polymers J. Polym. Sci.: Macromol. Rev. 16 245320

[8] Taleb M, Teyssedre G, Le Roy S and Laurent C 2013 Modelling of charge injection and extraction in a metal/polymer interface through an exponential distribution of surface states IEEE Trans. Dielectr. Electr. Insul. 20 311-320

[9] Blaise G 2001 Charge localization and transport in disordered dielectric materials J. Electrostatics 50 69-89

[10] Cubero D, Quirke N and Coker D F 2003 Electronic transport in disordered n-alkanes: from fluid methane to amorphous polyethylene J. Chem. Phys. 119 2669-2679

[11] Unge M and Christen T 2014 Electron and hole mobility edges in polyethylene from material simulations Chem. Phys. Lett. 613 15-18

[12] Le Roy S, Segur P, Laurent C and Teyssedre G 2004 Description of bipolar charge transport in polyethylene using a fluid model with a constant mobility: model prediction J. Phys. D: Appl. Phys. 37 298-305

[13] Meunier M, Quirke N and Aslanides A 2001 Molecular modeling of electron traps in polymer insulators: chemical defects and impurities J. Chem. Phys. 115 2876-2881

[14] Sessler G 1987 Physical principles of electrets Topics in Applied Physics: Electrets 33 13-80

[15] Mazzanti G, Marzinotto M and Battaglia A 2015 A first step towards predicting the life of HVDC cables subjected to load cycles and voltage polarity reversal Proc. 2015 IEEE Conf. Electrical Insulation and Dielectric Phenomena (CEIDP) 783-786

[16] Laurent C, Teyssedre G, Le Roy S and Baudoin F 2013 Charge dynamics and its energetic features in polymeric materials IEEE Trans. Dielectr. Electr. Insul. 20 357-381

[17] Chen L, Tran H D, Wang C C and Ramprasad R 2015 Unraveling the luminescence signatures of chemical defects in polyethylene J. Chem. Phys. 143124907

[18] Huzayyin A, Boggs S and Ramprasad R 2010 Quantum mechanical studies of carbonyl impurities in dielectric polyethylene IEEE Trans. Dielectr. Electr. Insul. 17 920-925

[19] Yang Z D, Zhang H, Zhao H and Han B Z 2015Trap mechanism based on frontier molecular orbitals of additives in polyethylene insulators: A theoretical study and molecular design strategy Internat. J. Quantum Chem. 115 1483-1489

[20] Teyssedre G, Laurent C, Campus A, Nilsson U H and Montanari G C 2003 Antioxidant and its reaction products as charge trapping centres in crosslinked polyethylene Proc. 2003 IEEE Int. Conf. Electrical Insulation and Dielectric Phenomena (CEIDP) 96-99

[21] Teyssedre G, Cisse L, Laurent C, Massines F and Tiemblo P 1998 Spectral analysis of optical emission due to isothermal charge recombination in polyolefins IEEE Trans. Dielectr. Electr. Insul. 5 527-535

[22] Kojima K, Maeda A, Takai Y and leda M 1978 Thermally stimulated currents from polyethylene terephthalate due to injected charges Jpn. J. Appl. Phys. 17 1735-1738

[23] Mizutani T, Suzuoki Y, Hanai M and leda M 1982 Determination of trapping parameters from TSC in polyethylene Jpn. J. Appl. Phys. 21 1639-1641

[24] leda M 1987 Carrier injection, space charge and electrical breakdown in insulating polymers IEEE Trans. Electr. Insul. 22 261-267 
[25] Blake A E, Charlesby A and Randle K J 1974 Simultaneous thermoluminescence and thermally stimulated conductivity in polyethylene J. Phys. D. 7, 759-770

[26] Mellinger A, Camacho Gonzalez F and Gerhard-Multhaupt R 2004 Photostimulated discharge in electret polymers: an alternative approach for investigating deep traps IEEE Trans. Dielectr. Electr. Insul. 11 218-226

[27] Zhu Z, Zhang $\mathrm{Y}, \mathrm{An} \mathrm{Z}$ and Zheng $\mathrm{F} 2012$ Investigation of space charge trap levels in $\mathrm{Al}_{2} \mathrm{O}_{3}$ nano-powder doped polyethylene by photo-stimulated discharge method Internat. J. Modern Phys. B 261250140

[28] Ma P, Zhang Y, Zheng F, An Z and Lei Q 2015 Investigation of surface and bulk charge traps of polyimide film by using the photo-stimulated discharge method Eur. Phys. Lett. 10927006

[29] Anta J A, Marcelli G, Meunier M and Quirke N 2002 Models of electron trapping and transport in polyethylene: Current-voltage characteristics J. Appl. Phys. 92 1002-1008

[30] Boufayed F, Teyssedre G, Laurent C, Le Roy S, Dissado L A, Ségur P and Montanari G C 2006 Models of bipolar charge transport in polyethylene J Appl. Phys. 100104105

[31] Damamme G, Le Gressus C and De Reggi A S 1997 Space charge characterization for the 21th century IEEE Trans. Dielectr. Electr. Insul. 4 558-584

[32] Haneef H F, Zeidell A M and Jurchescu O D 2020 Charge carrier traps in organic semiconductors: a review on the underlying physics and impact on electronic devices J. Mater. Chem. C 8 759-787

[33] Simmons J G and Tam M C 1973 Theory of isothermal currents and the direct determination of trap parameters in semiconductors and insulators containing arbitrary trap distributions Phys. Rev. 17 3706-3713

[34] Vivadi Y A, Barkhalov B S and Ragimov A V 1980 Isothermal depolarization currents in the organic semiconductor polynaphthol Thin Solid Films 66 L43-L45

[35] Watson P K 1990 The energy distribution of localized states in polystyrene, based on isothermal discharge measurements J. Phys. D: Appl. Phys. 23 1479-1484

[36] Molinié P 2012 A review of mechanisms and models accounting for surface potential decay IEEE Trans. Plasma Sci. 40 167-176

[37] Campos M and Giacometti J A 1981 Surface-potential decay in insulators with deep traps J. Appl. Phys. 52, 4546-4552

[38] Von Berlepsch H 1985 Interpretation of surface potential kinetics in HDPE by a trapping model J. Phys. D: Appl. Phys. 18 1155-1170

[39] Li J, Zhou F, Min D, Li S and Xia R 2015 The energy distribution of trapped charges in polymers based on isothermal surface potential decay model IEEE Trans. Dielectr. Electr. Insul. 22 1723-1732

[40] Wintle H J 1972 Surface-charge decay in insulators with nonconstant mobility and with deep trapping J. Appl. Phys. 43 2927-2930

[41] Dissado L A, Paris O, Ditchi T, Alquie C and Lewiner J 1999 Space charge Injection and extraction in high divergent fields Proc. IEEE Conf. Electrical Insulation Dielectric Phenomena (CEIDP) 23-26

[42] Mazzanti G, Montanari G C and Alison J M 2003 A space charge based method for the estimation of apparent mobility and trap depth as markers for insulation degradation-theoretical basis and experimental validation IEEE Trans. Dielectr. Electr. Insul. 10 187-197

[43] Dissado L A, Griseri V, Peasgood W, Cooper E S, Fukunaga K and Fothergill J C 2006 Decay of space charge in a glassy epoxy resin following voltage removal IEEE Trans. Dielectr. Electr. Insul. 13 903-916

[44] Laurent C, Massines F and Mayoux C 1997 Optical emission due to space charge effects in electrically stressed polymers IEEE Trans. Dielectr. Electr. Insul. 4 585-603

[45] Massines F, Tiemblo P, Teyssedre $G$ and Laurent C 1997 On the nature of the luminescence emitted by a polypropylene film after interaction with a cold plasma at low temperature J. Appl. Phys. 81 937-943

[46] George T and Randle K J 1975 The isothermal luminescence and thermoluminescence of gamma-irradiated polyethylene at 77 K J. Phys. D: Appl. Phys. 8 1585-1594

[47] Hama Y, Kimura Y, Tsumura M and Omi N 1980 Studies of the recombination of cation-electron pairs by long-range tunneling, as studied by ITL measurement in irradiated polymers Chem. Phys. 53 115-122

[48] He L J, Yang X, Niu H Q, Yuan Z H, Li D W and Chen C T 2016 Influence of photoconductivity on the study of trap levels in polyimide by photo-stimulated discharge Modern Phys. Lett. B 301650410

[49] Van Turnhout J 1987 Thermally stimulated discharge of electrets Topics in Applied Physics: Electrets 33 81-215

[50] Markiewicz A, Balbachas D V and Fleming R J 1991 Simultaneous thermally stimulated luminescence and depolarization current in low density polyethylene J. Thermal Analysis 37 1137-1152

[51] Hogue M D, Mucciolo ER and Calle C I 2007 Triboelectric, corona, and induction charging of insulators as a function of pressure J. Electrostatics 65 274-279

[52] Perlman M M 1972 Thermally stimulated currents and voltages and dielectric properties J. Electrochem. Soc. 119, 892-898

[53] Kao K J, Bamji S S and Perlman M M 1979 Thermally stimulated discharge current study of surface charge release in polyethylene by corona-generated excited molecules, and the crossover phenomenon J. Appl. Phys. 50 8181-8185 
Review: Trap Spectroscopy in Polymer Dielectrics - Revised Version. Teyssedre et al 2021 J. Phys. D: Appl. Phys. https://doi.org/10.1088/1361-6463/abf44a

[54] Teyssedre G, Tiemblo P, Massines F and Laurent C 1996 On the UV contribution to plasma induced luminescence of Polypropylene J. Phys. D: Appl. Phys. 29 3137-3146.

[55] Lavergne C and Lacabanne C 1993 A review of thermo-stimulated current", IEEE Electr. Insul. Mag. 9_2 5-21

[56] Correia N T, Alvarez C, Moura Ramos J J and Descamps M 2000 Molecular motions in molecular glasses as studied by thermally stimulated depolarisation currents (TSDC) Chem. Phys. 252 151-163

[57] Neagu E R and Neagu R 2002 The determination of relaxation parameters and their distributions using thermally stimulated discharge current measurements J. Phys. D: Appl. Phys. 35 2298-2303

[58] Sessler G M 1992 Charge dynamics in irradiated polymers IEEE Trans. Electr. Insul. 27 961-973

[59] Perrin C, Griseri V, Inguimbert C and Laurent C 2008 Analysis of internal charge distribution in electron irradiated polyethylene and polyimide films using a new experimental method J. Phys. D: Appl. Phys. 41205417

[60] Banda M E, Griseri V, Teyssedre G and Le Roy S 2018 Polarization of electron-beam irradiated LDPE films: contribution to charge generation and transport J. Phys. D: Appl. Phys. 51, 155303

[61] Yue L, Wang X, Wu Y, Cao J L, Liu Y, Sun C and Yang J 2013 Study on evolution of deep charge traps in polyimide irradiated by low-energy protons using photo-stimulated discharge technique J. Phys. D: Appl. Phys. 46, 145502

[62] Delbecq C J, Toyozawa Y and Yuster P 1974 Tunneling recombination of trapped electrons and holes in $\mathrm{KCl}: \mathrm{AgCl}$ and $\mathrm{KCl}: \mathrm{TCl}$ Phys. Rev. B 9 4497-4505

[63] Hagekyriakou J and Fleming R J 1982 Determination of the kinetic order of thermoluminescence in the presence of a distribution of electron trap activation energies J. Phys. D: Appl. Phys. 15 163-176

[64] Butlers P, Tale I, Pospisil J and Nespurek S 1988 Self-trapping of charge carriers in polymers: A comparative study of Poly(p-phenylene) and Poly(N-vinylcarbazole) Prog. Coll.\& Polym. Sci. 78, 93-96

[65] Cordier P, Delouis J F, Kieffer F, Lapersonne C and Rigaut J 1974 Etude cinétique de la luminescence différée isotherme d'un verre organique après impulsion d'électrons accélérés C. R. Hebd. Acad. Sci. C-279 589-591

[66] Charlesby A and Owen G P 1976 Luminescence enhancement in irradiated polyethylene Int. J. Radiat. Phys. Chem. 8 343-347

[67] Gheysari D and Behjat A 2001 Radiation crosslinking of LDPE and HDPE with 5 and $10 \mathrm{eV}$ electron beams Eur. Polym. J. 37 2011-2016

[68] Gheysari D, Behjat A and Haji-Saeid M 2001 The effect of high-energy electron beam on mechanical and thermal properties of LDPE and HDPE", .Eur. Polym. J. 37 295-302

[69] Murray K A, Kennedy J E, McEvoy B, Vrain O, Ryan D and Higginbotham C L 2012 The effects of high energy electron beam irradiation on the thermal and structural properties of low density polyethylene Radiat. Phys. Chem. 81 962-966

[70] Kawamura Y 2004 Effects of gamma irradiation on polyethylene, polypropylene, and polystyrene ACS Symposium Series 875 262-276

[71] Mariani M, Ravasio U, Varoli V, Consolati G, Faucitano A and Buttafava A 2007 Gamma irradiation of polyester films Radiat. Phys. Chem. 76 1385-1389

[72] Belkahla N, Teyssedre G, Saidi-Amroun N, Saidi M, Boudou L and Berquez L 2014 Space charge, conduction and photoluminescence measurements in gamma-irradiated Poly (ethylene-2,6-naphthalate) Radiat. Phys. Chem. 101 73-80

[73] Molinie P, Goldman M and Gatellet J 1995 Surface potential decay on corona-charged epoxy samples due to polarization processes J. Phys. D 28 1601-1620

[74] Mizutani T, Oomura T and leda M 1981 Surface potential decay in polyethylene Jpn. J. Appl. Phys. 20 855-859

[75] Sessler G M and Von Seggern H 1979 Crossover of surface potential decays in electron beam charged polyethylene Proc. 1979 Internat. Conf. Electrical Insulation and Dielectric Phenomena (CEIDP) 160-167

[76] Le Gressus C and Blaise G 1992 Insulator surface analysis J. Electron Spectroscop. Relat. Phenom. 59 73-96

[77] Molinié P 2005 Measuring and modeling transient insulator response to charging: The contribution of surface potential studies IEEE Trans. Dielectr. Electr. Insul. 12 939-950

[78] Zhang B and Zhang $G \times 2017$ Interpretation of the surface charge decay kinetics on insulators with different neutralization mechanisms J. Appl. Phys. 121105105

[79] Chen G 2010 A new model for surface potential decay of corona-charged polymers J. Phys. D: Appl. Phys. 43055405

[80] Coelho R, Levy L and Sarrail D 1986 On the natural decay of corona charged insulating sheets Phys. Stat. sol. a 94 289298

[81] leda M, Sawa G and Shinohara I 1967 A decay process of surface electric charges across polyethylene film J. Appl. Phys. 6 793-794

[82] Shen W W, Mu H B, Zhang G J, Deng J B and Tu D M 2013 Identification of electron and hole trap based on isothermal surface potential decay model J. Appl. Phys. 113083706

[83] Arkhipov V I, Popova J A, Rudenko A I and Sessler G M 1986 Space-charge perturbed dispersive transport in disordered dielectrics J. Electrostatics 18 23-37

[84] Tomaszewicz W 2001 Surface potential decay of disordered solids J. Electrostatics 51/52 340-344 
Review: Trap Spectroscopy in Polymer Dielectrics - Revised Version. Teyssedre et al 2021 J. Phys. D: Appl. Phys. https://doi.org/10.1088/1361-6463/abf44a

[85] Watson P K 1995 The transport and trapping of electrons in polymers IEEE Trans. Dielectr. Electr. Insul. 2 915-924

[86] Rychkov D, Kuznetsov A and Rychkov A 2011 Electret properties of polyethylene and polytetrafluoroethylene films with chemically modified surface IEEE Trans. Dielectr. Electr. Insul. 18 8-14

[87] Zhang B, Zhang G, Wang Q, Li C Y, He J L and An Z L 2015 Suppression of surface charge accumulation on Al2O3-filled epoxy resin insulator under dc voltage by direct fluorination AIP Advances 5127207

[88] Hillenbrand J, Motz T, Sessler G M, Zhang X, Behrendt N, Von Salis-Soglio C, Erhard D P, Altstadt V and Schmidt H W 2009 The effect of additives on charge decay in electron-beam charged polypropylene films J. Phys. D: Appl. Phys. 42 065410

[89] Gao Y, Li J, Yuan Y Q, Huang S H and Du B X 2018 Trap distribution and dielectric breakdown of isotactic polypropylene / propylene based elastomer with improved flexibility for DC cable insulation Access IEEE 6 58645-58661

[90] Li H, Zhai S, Hu L and Chen J 2019 The energy distribution of trapped charges on the surface of cross-linked polyethylene thermally aged at different temperatures J. Mater. Sci. Mater. Electron. 30 9015-9021

[91] Xing Z L , Zhang C, Cui H Z, Hai Y L, Wu Q Z and Min D M 2019 Space charge accumulation and decay in dielectric materials with dual discrete traps Appl. Sci. 94253

[92] Meng J, Zhang Y, Holé S, Zheng F and An Z 2017 Retrieval of charge mobility from apparent charge packet movements in LDPE thin films Europhysics Lett. 11767003

[93] Takada T, Miyake $\mathrm{H}$ and Tanaka Y 2006 Pulse acoustic technology for measurement of charge distribution in dielectric materials for spacecraft IEEE Trans. Plasma Sci. 34 2176-2184

[94] Lindmayer J 1965 Current transients in insulators J. Appl. Phys. 36 196-201

[95] Tzimas A, Rowland S M and Dissado L A 2012 Effect of electrical and thermal stressing on charge traps in XLPE cable insulation IEEE Trans. Dielectr. Electr. Insul. 19 2145-2154

[96] Vu T T N, Teyssedre G, Le Roy S and Laurent C 2017 Space charge criteria for the assessment of insulation materials for HVDC IEEE Trans. Dielectr. Electr. Insul. 24 1405-1415

[97] Mazzanti G, Montanari G C, Palmieri F and Alison J 2003 Apparent trap-controlled mobility evaluation in insulating polymers through depolarization characteristics derived by space charge measurements J. App Phys. 94 5997-6004

[98] Van der Born D, Tsekmes I A, Morshuis P H F, Smit J J, Person T J and Sutton S J 2013 Evaluation of apparent trapcontrolled mobility and trap depth in polymeric HVDC mini-cables Proc. 2013 IEEE Internat. Conf. Solid Dielectrics (ICSD) 242-245

[99] Haque N, Dalai S, Chatterjee B and Chakravorti S 2017 Investigations on charge trapping and de-trapping properties of polymeric insulators through discharge current measurements IEEE Trans. Dielectr. Electr. Insul. 24 583-591

[100] Zhou T, Chen G, Liao R and Xu Z 2011 Charge trapping and detrapping in polymeric materials: Trapping parameters J. Appl. Phys. 110043724

[101] Haque N, Dalai S, Chatterjee B and Chakravorti S 2016 Investigation of charge trapping behavior of LDPE using detrapping characteristics Proc. 2016 IEEE 7th Power India Internat. Conf. 1-5

[102] Montanari G C, Mazzanti G, Palmieri F, Motori A, Perego G and Serra S 2001 Space-charge trapping and conduction in LDPE, HDPE and XLPE J. Phys. D: Appl. Phys. 34 2902-2911

[103] Rogti F and Ferhat M 2015 Effect of temperature on trap depth formation in multi-layer insulation: Low density polyethylene and fluorinated ethylene propylene Appl. Phys. Lett. 104031605

[104] Teyssedre G, Laurent C, Perego G and Montanari G C 2009 Charge recombination induced luminescence of chemically modified cross-linked polyethylene materials IEEE Trans. Dielectr. Electr. Insul. 16 232-240

[105] Tiemblo P, Gomez-Elvira J M, Teyssedre G, Massines F and Laurent C 1999 Effect of a cold Helium plasma at $-180^{\circ} \mathrm{C}$ on polyolefin films: I. Plasma induced luminescence features of Polyethylene and Polypropylene Polymer Degrad. Stabil. 64 59-66

[106] Tiemblo P, Gomez-Elvira J M, Teyssedre G, Massines F and Laurent C 1999 Effect of a cold Helium plasma at $-180^{\circ} \mathrm{C}$ on polyolefin films: II. The chemiluminescence component Polymer Degrad. Stabil. 64 67-73

[107] Hagston W E 1975 Interpretation of electrophotoluminescence in terms of electron tunneling J. Phys. C: Solid State Phys. 9 647-661

[108] Jonscher A K and De Polignac A 1984 The time dependence of luminescence in solids J. Phys. C: Solid State Phys. 17 6493-6519

[109] Huntley D J 2003 An explanation of the power-law decay of luminescence J. Phys.: Condensed Matter 18 1359-1365

[110] Laurent C, Teyssedre G, Augé J L, Mazzanti G, Montanari G C, Dissado L A, Fothergill J C and See A 2000 Space charge and associated electroluminescence processes in XLPE cable peelings Proc. 2000 IEEE Internat. Conf. on Electrical Insulation and Dielectric Phenomena (CEIDP) 568-572

[111] Le Roy S, Teyssedre $G$ and Laurent $C 2005$ Charge transport and dissipative processes in insulating polymers: Experiments and model IEEE Trans. Dielectr. Electr. Insul. 12 644-654

[112] Cheug P S R, Roberts C W and Wagener K B 1979 Synthesis, photodegradation, and energy transfer in a series of poly(ethylene terephthalate-co-2,6-naphthalenedicarboxylate) copolymers J. Appl. Polym. Sci. 24 1809-1830 
Review: Trap Spectroscopy in Polymer Dielectrics - Revised Version. Teyssedre et al 2021 J. Phys. D: Appl. Phys. https://doi.org/10.1088/1361-6463/abf44a

[113] Nakamura H, Shirakawa Y, Takahashi S and Shimizu H 2011 Evidence of deep-blue photon emission at high efficiency by common plastic Europhysics Lett. 9522001

[114] Augé J L, Teyssedre G, Laurent C, Ditchi T and Holé S 2000 Combined electroluminescence and charge profile measurements in Poly(ethylene-2,6-naphthalate) under a DC field J. Phys. D : Appl. Phys. 33 3129-3138

[115] Tran Anh T, Berquez L, Boudou L and Martinez-Vega J 2011 Effect of trapped space charge on mechanical deformation induced by electric field IEEE Trans. Dielectr. Electr. Insul. 18, 1416-1422

[116] Teyssedre G, Mary D and Laurent C 1998 Analysis of the luminescence decay following excitation of Polyethylene Naphthalate films by an electric field J. Phys. D: Appl. Phys. 31 267-275

[117] Teyssedre G, Laurent C, Montanari G C, Palmieri F, See A, Dissado L A and Fothergill J C 2001 Charge distribution and electroluminescence in XLPE under DC field J. Phys. D: Appl. Phys. 34 2830-2844

[118] Mammeri M, Laurent C and Nedjar M 1997 Dynamics of voltage polarity reversal as the controlling factor in spacecharge induced breakdown of insulating polymers IEEE Trans. Dielectr. Electr. Insul. 4 44-51

[119] Bucci C, Fieschi R and Guidi G 1966 Ionic thermocurrents in dielectrics Phys. Rev. 148 816-823

[120] Vanderschueren J and Gasiot J 1979 Field-induced thermally stimulated currents in Thermally Stimulated Relaxation in Solids (P Bräunlich Berlin: Springer) Ch. 4, pp. 135-223

[121] Van Turnhout J 1971 Thermally stimulated discharge of polymer electrets Polymer J. 2 173-191

[122] Vanderschueren J, Linkens A, Gasiot J, Fillard J P and Parot P 1980 Simulation of field-induced thermally stimulated currents in dielectrics. Application to the dipolar case in polymeric systems J. Appl. Phys. 51 4967-4975

[123] Bräunlich P, Kelly P and Fillard J P 1979 Thermally stimulated luminescence and conductivity Thermally Stimulated Relaxation in Solids (P Bräunlich Berlin: Springer) Ch. 2, pp. 35-92

[124] Garlick G F J and Gibson A F 1948 The electron trap mechanism of luminescence in sulphide and silicate phosphors Proc. Royal Soc. London 60 574-590

[125] Teyssedre $\mathrm{G}$ and Lacabanne $\mathrm{C} 1995$ Some considerations about the analysis of thermostimulated depolarization peaks J. Phys. D: Appl. Phys. 28 1478-1487

[126] Crine J P 1990 Origin and significance of the compensation law in electrical conduction and dielectric relaxation of plasticized PVC J. Phys. D: Appl. Phys. 23 1315-1319

[127] Yelon A, Movaghar B and Crandall R S 2006 Multi-excitation entropy: its role in thermodynamics and kinetics Rep. Prog. Phys. 69 1145-1194

[128] Mansimov S A, Kerimov M K, Gezalov K B and Kovarskii A L 1986 Methods of investigation Analysis of the distribution of molecular motion with respect to relaxation times in polymers on the basis of thermostimulation of depolarization Polymer Science U.S.S.R. 28 2221-2226

[129] Neagu R M, Neagu E R, Kalogeras I M and Vassilikou-Dova A 2001 Evaluation of the dielectric parameters from TSDC spectra: Application to polymeric systems Mater. Res. Innov. 4 115-125

[130] Le Roy S, Baudoin F, Boudou L, Laurent C and Teyssedre G 2010 Thermo-stimulated depolarization currents in polyethylene films. Numerical simulations and experiments Proc. 2010 IEEE Internat. Conf. Solid Dielectrics (ICSD) 1-4

[131] Rytöluoto I, Niittymäki M and Lahti K 2019 Practical Notes on the measurement and interpretation of thermally stimulated discharge currents in biaxially oriented polypropylene Proc. 26th Nordic Insulation Symposium on Materials, Components and Diagnostics (NORD-IS 19) 64-69

[132] Mizutani T and leda M 1978 TSC from corona-charged high-density polyethylene and the effects of oxidation J. Phys. D: Appl. Phys. 11 185-191

[133] Suzuoki Y, Yasuda K, Mizutani T and leda M 1977 The influence of oxidation on thermally stimulated currents from trapped carriers in high density polyethylene J. Phys. D: Appl. Phys. 10 1985-1990

[134] Garankin J, Plukis A and Plukien R 2018 Identification of particles of ionizing radiation by the analysis of fluorescence pulse form of the thin PEN film scintillator IEEE Trans. Nucl. Sci. 65 739-743

[135] Nishitani T 1975 Simultaneous observation of the thermally stimulated current and the thermoluminescence in Polyethylene Jpn. J. Appl. Phys. 14 721-724

[136] Wintle H J 1990 Basic physics of insulators IEEE Trans. Dielectr. Electr. Insul. 25 27-44

[137] Wintle H J 2003 Charge motion in technical insulators: facts, fancies and simulations IEEE Trans. Dielectr. Electr. Insul. $10826-841$

[138] Ambrico P F, Ambrico M, Colaianni A, Schiavulli L, Dilecce G and De Benedictis S 2010 Thermoluminescence study of the trapped charge at an alumina surface electrode in different dielectric barrier discharge regimes J. Phys. D: Appl. Phys. 43325201

[139] Kirsh Y 1992 Kinetic analysis of thermoluminescence - Theoretical and practical aspects Phys. Stat. Sol. a 129 15-48

[140] Bos A J J 2007 Theory of thermoluminescence Radiat. Meas. 41, S45-S56

[141] Kivits P and Hagebeuk H J L 1977 Evaluation of the model for thermally stimulated luminescence and conductivity; reliability of trap depth determinations J. Luminescence 15 1-27 
Review: Trap Spectroscopy in Polymer Dielectrics - Revised Version. Teyssedre et al 2021 J. Phys. D: Appl. Phys. https://doi.org/10.1088/1361-6463/abf44a

[142] Chen R and Pagonis V 2014 The role of simulations in the study of thermoluminescence (TL) Radiat. Meas. 71 8-14

[143] Kirsh Y and Chen R 1991 Analysis of the blue phosphorescence of X-irradiated albite using a TL-like presentation Nuclear Tracks Radiat. Meas. 18 37-40

[144] Du J, De Clercq O Q and Poelman D 2019 Temperature dependent persistent luminescence: Evaluating the optimum working temperature Nature Sci. Reports 910517

[145] Kirsh Y 1991 The kinetics of the red and blue bands in the phosphorescence of X-irradiated albite Phys. stat. sol. a 127 265-273

[146] McKeever S W S and Chen R 1997 Luminescence models Radiat. Meas. 27 625-661

[147] Jain M, Guralnik B and Andersen M T 2012 Stimulated luminescence emission from localized recombination in randomly distributed defects J. Phys.: Condens. Matter 24385402

[148] Suzuoki Y, Yasuda K, Mizutani T and leda M 1977 Thermoluminescence in high density polyethylene and its oxidation effects Jpn. J. Appl. Phys. 16 1339-1342

[149] Brodribb J D, Hughes D M and Lewis T J 1973 The energy spectrum of traps in insulators by photon-induced current spectroscopy in Electrets, Charge Storage, and Transport in Dielectrics (M. Perlman, Electrochemical Society) 177-187

[150] Thomas J H and Feigl F J 1972 Electron trapping levels in silicon dioxide thermally grown on silicon J. Phys. Chem. Solids 33 2197-2216

[151] Brodribb J D, O'Colmain D and Hughes D M 1975 The theory of photon-stimulated current spectroscopy J. Phys. D: Appl. Phys. 8 856-862

[152] Zhu Z, Zhang Y, An Z and Zheng F 2011 Methodological investigation on photo-stimulated discharge to obtain accurate trap information in polymer dielectrics Meas. Sci. Technol. 22085109

[153] Ma P, Zhang Y, Holé S, Zheng F, Gua M and An Z 2016 Analysis and interpretation of photo-stimulated discharge spectrum for polypropylene films under different electric and geometrical conditions J. Electrostatics 84 128-134

[154] Zheng F, Li W, Gu M, An Z and Zhang Y 2017 Photo-stimulated discharge current in polyimide films IEEE Trans. Dielectr. Electr. Insul. 24 1802-1808

[155] Gunton W, Polovy G, Semczuk M and Madison K W 2006 Transparent electrodes for high E-field production using a buried indium tin oxide layer Rev. Sci. Instrum. 87033113

[156] Crine J P and Yelon A 1980 Photogeneration and transport of carriers in atactic polystyrene J. Appl. Phys. 51 21062114

[157] Takai Y, Osawa T, Mizutani T and leda M 1977 Photoconduction in poly(ethylene terephthalate). I. Mechanisms of carrier generation J. Polymer Sci.: Phys. Ed. 15 945-954

[158] Binks A E, Campbell A G and Scharples A 1970 Photocurrents in simple polymer systems J. Polymer Sci. A2 8 529-540

[159] Kan L and Kao K C 1993 Ultraviolet absorption and photoconduction spectra of polyimide films fabricated at various curing temperatures J. Chem. Phys. 98 3445-3451

[160] Ma P, Zhang Y, Holé S, Zheng F, Gu M and An Z 2016 Effect of photoemission from electrode on photo-stimulated discharge spectra Proc. 2016 IEEE Internat. Conf. on Dielectrics (ICD) 187-190

[161] Boudou L, Berquez L and Teyssedre G 2020 Combined-PSD and LIMM measurements for traps characterization in BoPP thin films Proc. 2020 IEEE Internat. Conf. on Dielectrics (ICD) 1-4

|162] Afanas'ev V V, Wang W C, Cerbu F, Madia O, Houssa M and Stesmans A 2014 Spectroscopy of deep gap states in high$\mathrm{k}$ insulators ECS Trans. 64 17-22

[163] Tydtgat C, Meert K W, Poelman D and Smet F 2016 Optically stimulated detrapping during charging of persistent phosphors Optical Materials Express 6 844-858

[164] He L J, Li D W, Wang D N, Li J, Wang X and Lei Q Q 2011 Study on the detrapping of charge carriers in polyethylene films, Proc. 2011 IEEE Internat. Conf. on Electrical Insulation and Dielectric Phenomena (CEIDP) 264-266

[165] Boudou L, Zheng F and Teyssedre G 2018 Photo-stimulated discharge current measurements on biaxially oriented polypropylene thin films (BOPP) Proc. Internat. Conf. Properties Applications of Dielectric Materials (ICPADM) 722-725

[166] McKeever S W S 2001 Optically stimulated luminescence dosimetry Nucl. Instr. Meth. Phys. Rev. B 184 29-54

[167] Yukihara E G, McKeever S W S and Akselrod M S 2014 State of art: Optically stimulated luminescence dosimetry Frontiers of future research Radiat. Meas. 71 15-24

[168] McKeever S W S, Bøtter-Jensen L, Larsen N A and Duller G A T 1997 Temperature dependence of OSL decay curves: experimental and theoretical aspects Radiat. Meas. 27 161-170

[169] Bailiff I K and Poolton N R J 1991 Studies of charge transfer mechanisms in feldspars Nucl. Tracks Radiat. Meas. 18 111-118

[170] Poolton N R J, Ozanyan K B, Wallinga J, Murray A S and Bøtter-Jensen L 2002 Electrons in feldspar II: a consideration of the influence of conduction band-tail states on luminescence processes Phys. Chem. Min. 29 217-225

[171] Jain M and Ankjærgaard C 2011 Towards a non-fading signal in feldspar: Insight into charge transport and tunnelling from time-resolved optically stimulated luminescence Radiat. Meas. 46 292-309 
[172] You F, Bos A J J, Shi Q F, Huang S and Dorenbos P 2012 Thermoluminescence investigation of donor $\left(\mathrm{Ce}^{3+}, \mathrm{Pr}^{3+}, \mathrm{Tb}^{3+}\right)$ acceptor $\left(\mathrm{Eu}^{3+}, \mathrm{Yb}^{3+}\right)$ pairs in $\mathrm{Y}_{3} \mathrm{Al}_{5} \mathrm{O}_{12}$ Phys. Rev. B 85115101

[173] Laredo E, Bello A and Grimau M 1999 The analysis of TSDC peaks with a KWW relaxation function or a distribution of relaxation times in polymers Polymer Bulletin 42 117-124

[174] Alegría A, Goitiandia L and Colmenero J 2000 Interpretation of the TSDC fractional polarization experiments on the $\alpha$ relaxation of polymers J. Polym. Sci. B: Polym. Phys. 38 2105-2113

[175] Viciosa M T, Pires G and Moura Ramos J J 2009 Is the Kohlrausch function a good tool to account for nonexponentiality in thermally stimulated depolarisation currents (TSDC) data treatment? Chem. Phys. 359 156-160

[176] Tian F, Bu W, Shi L, Yang C, Wang Y and Lei Q 2011 Theory of modified thermally stimulated current and direct determination of trap level distribution J. Electrostatics 69 7-10

[177] Lacabanne C, Chatain D, Guillet J, Seytre G and May J F 1975 Dielectric and depolarization thermocurrent study of the $\checkmark$ relaxation in polyethylene isophthalamide J. Polym. Sci.: Polym. Phys. Edn. 13 445-453

[178] Zieliński M, Swiderski T and Kryszewski M 1978 Thermal sampling in polymers with distributed relaxations: PMMA Polymer 19 883-888

[179] Chen R, Pagonis V and Lawless J L 2016 Evaluated thermoluminescence trapping parameters-What do they really mean? Radiat. Meas. 91 21-27

[180] Van den Eeckhout K, Bos A J J, Poelman D and Smet P F 2013 Revealing trap depth distributions in persistent phosphors Phys. Rev. B 87045126

[181] Dobruchowska E, Okrasa L, Glowacki I, Ulanski J and Boiteux G 2004 The 'wet dog' effect in polymers as seen by thermoluminescence Polymer 45 6027-6035

[182] Gonzalez FC, Mellinger A, Gerhard-Multhaupt R, Szamelt Z, Glowacki I and Ulanski J 2005 Thermoluminescence of poled polyethylene terephthalate films Proc. 12th International Symposium on Electrets (ISE) 288-291

[183] Somersall A C, Dan E and Guillet J E 1974 Photochemistry of ketone polymers. XI. Phosphorescence as a probe of subgroup motion in polymers at low temperatures Macromolecules 7 233-244

[184] Teyssedre G, Menegotto J and Laurent C 2001 Temperature dependence of the photoluminescence in Poly(ethylene terephthalate) Polymer $\mathbf{4 2}$ 8207-8216

[185] Light R R and Seymour R W 1982 Effect of sub-Tg relaxations on the gas transport properties of polyesters Polym. Engineering Sci. 22 857-864

[186] Fleming R J 1990 Thermally stimulated luminescence and conductivity — Additional tools for thermal analysis of polymers J. Thermal Analysis 36 331-351

[187] Charlesby A and Partridge R H 1965 The identification of luminescence centres in polyethylene and other polymers Proc. Roy. Soc. London A 283 312-328

[188] Teyssedre G, Tardieu G and Laurent C 2002 Characterization of crosslinked polyethylene materials by luminescence techniques J. Materials Sci. 37 1599-1609

[189] Balbachas D V and Fleming R J 1992 Thermally simulated conductivity and luminescence in cross-linked polyethylene Proc. 1992 IEEE Internat. Conf. Electrical Insulation and Dielectric Phenomena (CEIDP) 93-98

[190] Suzuoki Y, Mizutani T, Takai Y and leda M 1977 Investigation of carrier traps in polyethylene by PSDC and TSC Jpn. J. Appl. Phys. 16 1929-1931

[191] Grimmeiss H G and Ledebo L A 1975 Photo-ionization of deep impurity levels in semiconductors with non-parabolic bands J. Phys. C: Solid State Phys. 8 2615-2626

[192] Grimmeiss H G and Ledebo L A 1975 Spectral distribution of photoionization cross sections by photoconductivity measurements J. Appl. Phys. 46 2155-2162

[193] Duke C B and Schein L B 1980 Organic solids: is energy-band theory enough? Physics Today 33_2 42-48

[194] Brédas J L and Street G B 1985 Electronic structure of polystyrene: A valence effective Hamiltonian theoretical study J. Chem. Phys. 82 3284-3288

[195] Botelho do Rego A, Rei Vilar M, Lopes da Silva J, Heyman M and Schott M 1986 Electronic excitation and secondary electron emission studies by low-energy electrons backscattered from thin polystyrene film surfaces Surface Sci. 178 367-374

[196] Secu M and Polosan S 2018 Charge carrier traps in tris-(8-hydroxyquinoline) aluminum J. Luminescence 194 91-95

[197] Fischer P and Röhl P 1976 Thermally stimulated and isothermal depolarization currents in low-density polyethylene $J$. Polym. Sci.: Phys. Ed. 14 531-542

[198] Fischer P and Röhl P 1976 Relaxation time spectrum of dipolar reorientation in low-density polyethylene J. Polym. Sci.: Phys. Ed. 14 543-554

[199] Hoang A T, Serdyuk Y V and Gubanski S M 2020 Charging and discharge currents in low-density polyethylene and its nanocomposite Energies 131518

[200] Allen N S, Homer J and McKellar J F 1977 Origin and role of the luminescent species in the photo-oxidation of commercial polypropylene J. Appl. Polym. Sci. 21 2261-2267 
Review: Trap Spectroscopy in Polymer Dielectrics - Revised Version. Teyssedre et al 2021 J. Phys. D: Appl. Phys. https://doi.org/10.1088/1361-6463/abf44a

[201] Allen N S, Homer J and McKellar J F 1977 Identification of the luminescent species in low-density polyethylene J. Appl. Polym. Sci. 21 3147-3152

[202] Jacques P P L and Poller R C 1993 Fluorescence of polyolefins-2. Use of model compounds to identify fluorescent species in thermally degraded polymers Eur. Polym. J. 29 83-89

[203] Ito T, Fuse $\mathrm{N}$ and Ohki Y 2004 Effects of additives, photodegradation, and water-tree degradation on the photoluminescence in polyethylene and polypropylene IEEJ Trans. Fundamentals and Materials 124 624-630

[204] Zlatkevitch L 1989 Luminescence techniques in solid state polymer research (New York: Marcel Dekker Inc)

[205] Mousseron-Canet M and Mani J C 1969 Photochimie et réactions moléculaires (Paris: Dunod)

[206] Becker R S 1969 Theory and interpretation of fluorescence and phosphorescence, (New York: Wiley)

[207] Van Turnhout J 1972 Thermally stimulated discharge of polymer electrets PhD Thesis, Delft University

[208] Tardieu G, Teyssedre $G$ and Laurent C 2002 Role of additives as recombination centres in polyethylene materials as probed by luminescence techniques J. Phys. D: Appl. Phys. 35 40-47

[209] Peruzzotti F, Martinotto L and Del Brenna M 2004 Cable, in particular for transport or distribution of electrical energy and insulating composition US Patent 6696154

[210] Englund V, Hjertberg T, Huuva R and Gubanski S M 2007 Voltage stabilisers for XLPE in high voltage applications Proc. 2007 IEEE Internat. Conf. Solid Dielectrics (ICSD) 203-206

[211] Koyanagi M, Zwarich R J and Goodman L 1972 Phosphorescence spectrum of acetophenone: An example of pseudoJahr-Teller distortion J. Chem. Phys. 56, 3044-3060

[212] Matthews T G and Fytle F E 1979 Luminescence properties of highly purified acetophenone J. Luminescence $2193-99$

[213] Lamola A A 1967 Lowest $n-\pi *$ triplet state of acetophenone J. Chem. Phys. 47 4810-4816

[214] Yang N C, McClure D S, Murov S L, Houser J J and Dusenbery R 1967 Photoreduction of acetophenone and substituted acetophenones J. Am. Chem. Soc. 89 5466-5468

[215] Montanari G C, Laurent C, Teyssedre G, Campus A and Nilsson U H 2005 From LDPE to XLPE: investigating the change of electrical properties. Part 1: Space charge, conduction and life IEEE Trans. Dielectr. Electr. Insul. 12 438-446

[216] Kalinowski J 1997 Electronic processes in organic electroluminescence in Organic electroluminescent materials and devices (Miyata S and Nalwa H R New York: Gordon) Chap. 1, pp. 1-72

[217] Allen N S and McKellar J F 1978 Photochemical reactions in commercial poly(ethylene 2,6-naphthalate) J. Appl. Polym. Sci. 22 2085-2092

[218] Mary D, Albertini M and Laurent C 1997 Understanding optical emissions from electrically stressed insulating polymers: electro-luminescence in poly(ethylene terephthalate) and poly(ethylene 2, 6-naphthalate) films J. Phys. D.: Appl. Phys. 30 171-184

[219] Teyssedre G, Laurent C, Boudou L and Le Roy S 2019 High electric field behavior and energetic processes in Poly(ethylene naphthalate) Proc. 2019 IEEE Internat. Conf. Electrical Materials and Power Equipment (ICEMPE) 88-93

[220] Teyssedre G and Laurent C 1999 Tunneling-mediated recombination-induced luminescence in Polyethylene naphthalate Proc. of SPIE 4017 112-118

[221] Teyssedre G, Mary D, Laurent C and Mayoux C 1998 Optical emission due to space charge recombination in insulating polymers: An insight into electrical ageing", in Space charge in solid dielectrics (Fothergill J C and Dissado L A Leicester: Dielectrics Society) 285-302

[222] Brédas J L, Beljonne D, Coropceanu V and Cornil J 2004 Charge-transfer and energy-transfer processes in $\pi$-conjugated oligomers and polymers: a molecular picture Chem. Rev. 104 4971-5003

[223] Arden W, Kotani M and Peter L M 1976 Triplet exciton decay processes in crystalline tetracene Phys. Stat. Sol. $b 75$ 621-631

[224] Baldo M A, Adachi C and Forrest S R 2000 Transient analysis of organic electro-phosphorescence. II. Transient analysis of triplet-triplet annihilation Phys. Rev. B 6210967

[225] Mi R, Xing Z, Hao J, Hu X, Min D, Li S and Wu Q 2020 Effect of morphology and traps on DC conductivity and breakdown of polyethylene nanocomposites IEEE Trans. Dielectr. Electr. Insul. 27 489-497

[226] Hoang M Q, Boudou L, Le Roy S and Teyssedre G 2014 Dissociating space charge processes from orientation polarization in Poly(ethylene naphthalate) films J. Phys. D: Appl. Phys. 47455306

[227] Chen L, Batra R, Ranganathan R, Sotzing G, Cao Y and Ramprasad R 2018 Electronic structure of polymer dielectrics: the role of chemical and morphological complexity Chem. Mater. 30 7699-7706

[228] Kim S W, Suzuki K, Sugie A, Yoshida H, Yoshida M and Suzuki Y 2018 Effect of end group of amorphous perfluoropolymer electrets on electron trapping Sci. Technol. Adv. Mater. 19 486-494

[229] Takada T, Kikuchi H, Miyake H, Tanaka Y, Yoshida M and Hayase Y 2015 Determination of charge-trapping sites in saturated and aromatic polymers by quantum chemical calculation IEEE Trans Dielectr Electr Insul. 22 1240-1249

[230] Shandilya A, Schadler L S and Sundararaman R 2020 First-principles identification of localized trap states in polymer nanocomposite interfaces J. Materials Res. 35 931-939 
Review: Trap Spectroscopy in Polymer Dielectrics - Revised Version. Teyssedre et al 2021 J. Phys. D: Appl. Phys. https://doi.org/10.1088/1361-6463/abf44a

[231] Borgani R, Pallon L K H, Hedenqvist M S, Gedde U W and Haviland D B 2016 Local charge injection and extraction on surface-modified $\mathrm{Al}_{2} \mathrm{O}_{3}$ nanoparticles in LDPE Nano Lett. 16 5934-5937

[232] Leng X, Jin F, Wei M and Ma Y 2016 GW method and Bethe-Salpeter equation for calculating electronic excitations WIREs Comput. Mol. Sci. 6 532-550

[233] Roma G, Bruneval F and Martin-Samos L 2018 Optical properties of saturated and unsaturated carbonyl defects in polyethylene J. Phys. Chem. 8 2023-2030 